Studies on $\mathrm{K}_{2} \mathrm{CO}_{3}$-Catalyzed 1, 4-Addition of 1, 2-Allenic Ketones with Diethyl Malonate:

Controlled Selective Synthesis of $\beta, \gamma$-Unsaturated Enones and $\alpha$-Pyrones

Shengming Ma*, Shichao Yu, and Shaohu Yin

State Key Laboratory of Organometallic Chemistry, Shanghai Institute of Organic Chemistry,

Chinese Academy of Sciences, 354 Fenglin Lu, Shanghai 200032, P. R. China E-mail: masm@pub.sioc.ac.cn Fax: (+86) 21-64167510

Supporting Information

Table of Contents

\begin{tabular}{|c|c|}
\hline Analytical data for compounds $\mathbf{1 , 3}, \mathbf{4}$ and $\mathbf{6}$ & $\mathrm{S} 2$ \\
\hline${ }^{1} \mathrm{H}$ NMR and ${ }^{13} \mathrm{C}$ NMR spectra of those compounds & $\mathrm{S} 17$ \\
\hline
\end{tabular}


Synthesis of 9-butylhexadeca-9, 10-dien-8-one (1m): The reaction of oct-1-en-3-yne (2.16 g, $22 \mathrm{mmol}$ ) with $n$-BuLi (2.5 M in cyclohexane, $8 \mathrm{~mL}, 20 \mathrm{mmol})$ and $\mathrm{N}, \mathrm{N}$-dimethyl octamide (5.14 g, $30 \mathrm{mmol})$ afforded $3.24 \mathrm{~g}(54 \%)$ of 1m: liquid; IR(neat) 1944, 1678, 1466, 1089, 726 $\mathrm{cm}^{-1} ;{ }^{1} \mathrm{H}$ NMR $\left(300 \mathrm{MHz}, \mathrm{CDCl}_{3}\right): \delta 5.47-5.54(\mathrm{~m}, 1 \mathrm{H}), 2.57(\mathrm{t}, J=7.5 \mathrm{~Hz}, 2 \mathrm{H}), 2.06-2.19(\mathrm{~m}$, $4 \mathrm{H}), 1.17-1.60(\mathrm{~m}, 20 \mathrm{H}), 0.79-0.97(\mathrm{~m}, 9 \mathrm{H}) ;{ }^{13} \mathrm{C} \mathrm{NMR}\left(75 \mathrm{MHz}, \mathrm{CDCl}_{3}\right): \delta 211.7,201.8$, $108.9,95.1,39.1,31.6,31.3,30.1,29.2,29.0,28.7,28.3,26.3,25.2,22.5,22.3,22.2,13.9,13.9$, 13.8; MS m/z (\%) $292\left(\mathrm{M}^{+}, 2.48\right), 57$ (100); HRMS m/z (EI) calcd for $\mathrm{C}_{20} \mathrm{H}_{36} \mathrm{O} 292.27661$. Found 292.27694.

Synthesis of 2-butyl-1-phenylnona-2, 3-dien-1-one (1n): The reaction of oct-1-en-3-yne $(2.16 \mathrm{~g}, 22 \mathrm{mmol})$ with $n$-BuLi $(2.5 \mathrm{M}$ in cyclohexane, $8 \mathrm{~mL}, 20 \mathrm{mmol})$ and $\mathrm{N}, \mathrm{N}$-dimethyl benzamide (4.48 g, $30 \mathrm{mmol}$ ) afforded $3.89 \mathrm{~g}$ (72\%) of 1n: liquid; IR(neat) 3058, 1943, 1651, 1598, 1467, 1274, 1072, 941, $698 \mathrm{~cm}^{-1} ; \quad{ }^{1} \mathrm{H}$ NMR (300 MHz, $\left.\mathrm{CDCl}_{3}\right): \delta 7.69(\mathrm{~d}, J=5.4 \mathrm{~Hz}, 2$ H), 7.39-7.45 (m, $1 \mathrm{H})$, 7.29-7.35 (m, $2 \mathrm{H})$, 5.30-5.39 (m, $1 \mathrm{H})$, 2.35-2.42 (m, $2 \mathrm{H}), 1.95-2.12$ (m, $2 \mathrm{H}), 1.10-1.55(\mathrm{~m}, 10 \mathrm{H}), 0.78-0.98(\mathrm{~m}, 6 \mathrm{H}) ;{ }^{13} \mathrm{C} \mathrm{NMR}\left(75 \mathrm{MHz}, \mathrm{CDCl}_{3}\right): \delta 213.5,195.7$, $139.1,131.8,129.0,127.9,107.6,95.2,31.3,30.5,28.9,28.7,28.1,22.6,22.6,14.2,14.1$; MS $m / z(\%) 270\left(\mathrm{M}^{+}, 3.87\right), 105(100)$; HRMS m/z (EI) calcd for $\mathrm{C}_{19} \mathrm{H}_{26} \mathrm{O}$ 270.19837. Found 270.19621.

Synthesis of 2-butyl-1-p-tolylnona-2, 3-dien-1-one (10): The reaction of oct-1-en-3-yne (2.16 $\mathrm{g}, 20 \mathrm{mmol})$ with $n$-BuLi $(2.5 \mathrm{M}$ in cyclohexane, $8 \mathrm{~mL}, 20 \mathrm{mmol})$ and $\mathrm{N}$, N-dimethyl $p$ methylbenzamide (4.08 g, $25 \mathrm{mmol}$ ) afforded $4.20 \mathrm{~g}$ (74\%) of 1o: liquid; IR (neat): 1944, 1651, 1609, $1275 \mathrm{~cm}^{-1}$; ${ }^{1} \mathrm{H}$ NMR $\left(300 \mathrm{MHz}, \mathrm{CDCl}_{3}\right) \delta 7.65(\mathrm{~d}, J=7.8 \mathrm{~Hz}, 2 \mathrm{H}), 7.16(\mathrm{~d}, J=7.8 \mathrm{~Hz}$, $2 \mathrm{H})$, 5.40-5.30 (m, $1 \mathrm{H}), 2.44-2.30$ (m, $2 \mathrm{H}), 2.36$ (s, $3 \mathrm{H})$, 2.16-2.00 (m, $2 \mathrm{H}), 1.54-1.30$ (m, 6 
$\mathrm{H}), 1.30-1.14(\mathrm{~m}, 4 \mathrm{H}), 0.95(\mathrm{t}, J=6.9 \mathrm{~Hz}, 3 \mathrm{H}), 0.86(\mathrm{t}, J=6.9 \mathrm{~Hz}, 3 \mathrm{H}) ;{ }^{13} \mathrm{C} \mathrm{NMR}(75 \mathrm{MHz}$, $\left.\mathrm{CDCl}_{3}\right): \delta 212.8,195.3,142.1,136.1,129.0,128.3,128.3,107.1,94.7,94.7,31.1,30.2,28.6$, 28.4, 27.9, 22.3, 21.5, 21.4, 13.9; MS m/z (\%) $284\left(\mathrm{M}^{+}, 44.93\right), 119$ (100); Anal. calcd for $\mathrm{C}_{20} \mathrm{H}_{28} \mathrm{O}$ C 84.45, H 9.92. Found C 84.49, H 9.91.

Synthesis of 3-pentyldeca-3, 4-dien-2-one (1p): The reaction of nona-1-en-3-yne (1.34 g, 11 mmol) with $n$-BuLi (2.0 M in cyclohexane, $10 \mathrm{mmol})$ and $\mathrm{N}, \mathrm{N}$-dimethyl benzamide $(1.04 \mathrm{~g}$, $12 \mathrm{mmol}$ ) afforded $1.27 \mathrm{~g}$ (57\%) of 1p: liquid; IR (neat) 1944, $1679 \mathrm{~cm}^{-1} ;{ }^{1} \mathrm{H}$ NMR (300 MHz, $\left.\mathrm{CDCl}_{3}\right) \delta 5.56-5.50(\mathrm{~m}, 1 \mathrm{H}), 2.23(\mathrm{~s}, 3 \mathrm{H}), 2.10-2.20(\mathrm{~m}, 4 \mathrm{H}), 1.52-1.20(\mathrm{~m}, 12 \mathrm{H}), 0.92-0.80$ $(\mathrm{m}, 6 \mathrm{H}) ;{ }^{13} \mathrm{C} \mathrm{NMR}\left(75 \mathrm{MHz}, \mathrm{CDCl}_{3}\right) \delta 212.4,199.4,109.7,95.4,31.4,31.3,28.7,28.2,27.6$, 26.8, 26.4, 22.4, 22.3, 14.0, 13.9; MS m/z (\%) $222\left(\mathrm{M}^{+}, 1.69\right), 43$ (100); HRMS m/z (EI) calcd for $\mathrm{C}_{15} \mathrm{H}_{26} \mathrm{O} 222.1984$. Found 222.1949.

Synthesis of 9-pentylhexadeca-9, 10-dien-8-one (1q): The reaction of nona-1-en-3-yne (2.68 g, $22 \mathrm{mmol}$ ) with $n$-BuLi (2.5 M in cyclohexane, $8 \mathrm{~mL}, 20 \mathrm{mmol}$ ) and $\mathrm{N}$, N-dimethyl octamide $(5.14 \mathrm{~g}, 30 \mathrm{mmol})$ afforded $3.62 \mathrm{~g}(59 \%)$ of 1q: liquid; IR(neat) 1944, 1678, 1466, 1092, 726 $\mathrm{cm}^{-1} ;{ }^{1} \mathrm{H}$ NMR $\left(300 \mathrm{MHz}, \mathrm{CDCl}_{3}\right): \delta 5.46-5.51(\mathrm{~m}, 1 \mathrm{H}), 2.57(\mathrm{t}, J=7.5 \mathrm{~Hz}, 2 \mathrm{H}), 2.12(\mathrm{q}, J=$ 7.1 Hz, 4 H), 1.49-1.60 (m, 2 H), 1.40-1.49 (m, 2 H), 1.18-1.39 (m, 18 H), 0.80-0.92 (m, $9 \mathrm{H})$; ${ }^{13} \mathrm{C} \mathrm{NMR}\left(75 \mathrm{MHz}, \mathrm{CDCl}_{3}\right): \delta 211.7,202.1,109.5,95.1,39.1,31.6,31.33,31.28,29.2,29.0$, 28.7, 28.3, 27.6, 26.5, 25.2, 22.5, 22.37, 22.31, 13.91, 13.89, 13.85; MS $m / z(\%) 306\left(\mathrm{M}^{+}, 2.55\right)$, 57 (100); HRMS m/z (EI) calcd for $\mathrm{C}_{21} \mathrm{H}_{38} \mathrm{O}$ 306.29226. Found 306.28954.

Synthesis of 2-pentyl-1-phenylnona-2, 3-dien-1-one (1r): The reaction of nona-1-en-3-yne $(2.70 \mathrm{~g}, 22 \mathrm{mmol})$ with $n$-BuLi $(2.5 \mathrm{M}$ in cyclohexane, $8 \mathrm{~mL}, 20 \mathrm{mmol})$ and $\mathrm{N}, \mathrm{N}$-dimethyl benzamide (4.48 g, $30 \mathrm{mmol}$ ) afforded $4.32 \mathrm{~g}$ (76\%) of 1r: liquid; IR(neat) 3058, 1944, 1651, 
1598, 1578, 1447, 1270, 1072, 698, $648 \mathrm{~cm}^{-1} ;{ }^{1} \mathrm{H}$ NMR $\left(300 \mathrm{MHz}, \mathrm{CDCl}_{3}\right): \delta 7.70(\mathrm{~d}, J=6.9$ $\mathrm{Hz}, 2 \mathrm{H}), 7.47$ (t, $J=7.5 \mathrm{~Hz}, 1 \mathrm{H}), 7.37$ (t, $J=7.5 \mathrm{~Hz}, 2 \mathrm{H}), 5.31-5.40$ (m, $1 \mathrm{H}), 2.30-2.45(\mathrm{~m}$, $2 \mathrm{H}), 2.00-2.11(\mathrm{~m}, 2 \mathrm{H}), 1.04-1.54(\mathrm{~m}, 12 \mathrm{H}), 0.79-0.98(\mathrm{~m}, 6 \mathrm{H}) ;{ }^{13} \mathrm{C} \mathrm{NMR}\left(75 \mathrm{MHz}, \mathrm{CDCl}_{3}\right)$ : $\delta 213.6,196.0,139.2,131.8,129.0,127.9,107.7,95.2,31.7,31.4,28.9,28.7,28.3,28.0,22.8$, 22.6, 14.3, 14.2; MS m/z (\%) $284\left(\mathrm{M}^{+}, 4.01\right), 105$ (100); HRMS m/z (EI) calcd for $\mathrm{C}_{20} \mathrm{H}_{28} \mathrm{O}$ 284.21402. Found 284.21343.

Synthesis of dodeca-1, 2-dien-4-one (1v): liquid; IR(neat) 3066, 1961, 1934, 1684, 1159, 846, $722 \mathrm{~cm}^{-1} ;{ }^{1} \mathrm{H}$ NMR $\left(300 \mathrm{MHz}, \mathrm{CDCl}_{3}\right): \delta 5.75(\mathrm{t}, J=6.45 \mathrm{~Hz}, 1 \mathrm{H}), 5.21(\mathrm{~d}, J=6.45 \mathrm{~Hz}, 2 \mathrm{H})$, $2.58(\mathrm{t}, J=7.33 \mathrm{~Hz}, 2 \mathrm{H}), 1.55(\mathrm{t}, J=7.33 \mathrm{~Hz}, 2 \mathrm{H}), 1.08-1.38(\mathrm{~m}, 10 \mathrm{H}), 0.84(\mathrm{t}, J=6.45 \mathrm{~Hz}$, $3 \mathrm{H}) ;{ }^{13} \mathrm{C} \mathrm{NMR}\left(75 \mathrm{MHz}, \mathrm{CDCl}_{3}\right): \delta 216.8,201.5,96.9,79.5,39.4,32.0,29.6,29.4,29.3,24.9$, 22.9, 14.3; MS m/z (\%) $180\left(\mathrm{M}^{+}, 3.28\right), 141$ (100); HRMS m/z (EI) calcd for $\mathrm{C}_{12} \mathrm{H}_{20} \mathrm{O}$ 180.15141. Found 180.14840.

Synthesis of ethyl 2-(ethoxycarbonyl)-3-methyl-4-hexyl-5-oxo-2-hexenoate (4c) and 3(ethoxycarbonyl)-4, 6-dimethyl-5-hexyl-2-pyranone (6c): The reaction of 1c (185.1 mg, 1.2 $\mathrm{mmol}), \mathrm{K}_{2} \mathrm{CO}_{3}(14.1 \mathrm{mg}, 0.1 \mathrm{mmol})$ and diethyl malonate $(162.7 \mathrm{mg}, 1.0 \mathrm{mmol})$ in $2 \mathrm{ml}$ of acetone afforded $217.4 \mathrm{mg}(67 \%)$ of $\mathbf{4 c}$ and $76.0 \mathrm{mg}(27 \%)$ of $\mathbf{6 c} . \mathbf{4 c :}$ liquid; IR(neat) 2257, 1720, 1628, 1229, 1059, 910, $734 \mathrm{~cm}^{-1} ;{ }^{1} \mathrm{H}$ NMR (300 MHz, $\left.\mathrm{CDCl}_{3}\right): \delta 4.08-4.27(\mathrm{~m}, 5 \mathrm{H})$, 2.11(s, $3 \mathrm{H}), 1.81(\mathrm{~s}, 3 \mathrm{H}), 1.74-1.79(\mathrm{~m}, 1 \mathrm{H}), 1.38-1.50(\mathrm{~m}, 1 \mathrm{H}), 1.02-1.30(\mathrm{~m}, 14 \mathrm{H}), 0.76-$ $0.82(\mathrm{~m}, 3 \mathrm{H}) ;{ }^{13} \mathrm{C}$ NMR $\left(75 \mathrm{MHz}, \mathrm{CDCl}_{3}\right): \delta 206.7,165.5,165.2,153.7,128.0,61.4,61.3$, 57.5, 31.7, 29.6, 29.4, 28.3, 27.3, 22.7, 16.4, 16.4, 14.19, 14.18; MS m/z (\%) $326\left(\mathrm{M}^{+}, 0.97\right), 43$ (100); HRMS m/z (EI) calcd for $\mathrm{C}_{18} \mathrm{H}_{30} \mathrm{O}_{5}$ 326.20932. Found 326.20903. 6c: liquid; IR(neat) 2253, 1732, 1635, 1552, 1467, 1236, 1030, $732 \mathrm{~cm}^{-1} ;{ }^{1} \mathrm{H}$ NMR (300 MHz, $\left.\mathrm{CDCl}_{3}\right): \delta 4.26-4.33$ 
(m, $2 \mathrm{H}), 2.24-2.39(\mathrm{~m}, 2 \mathrm{H}), 2.20(\mathrm{~s}, 3 \mathrm{H}), 2.12(\mathrm{~s}, 3 \mathrm{H}), 1.09-1.40(\mathrm{~m}, 11 \mathrm{H}), 0.78-0.85$ (m, 3 $\mathrm{H}) ;{ }^{13} \mathrm{C}$ NMR $\left(75 \mathrm{MHz}, \mathrm{CDCl}_{3}\right): \delta 165.7,159.5,158.9,155.3,118.4,116.6,61.9,31.7,29.7$, 29.4, 27.1, 22.8, 17.9, 17.4, 14.3, 14.2; MS m/z (\%) $280\left(\mathrm{M}^{+}, 18.15\right), 84$ (100); HRMS m/z (EI) calcd for $\mathrm{C}_{16} \mathrm{H}_{24} \mathrm{O}_{4}$ 280.16746. Found 280.16380.

Synthesis of ethyl 2-(ethoxycarbonyl)-3-methyl-4-benzyl-5-oxo-2-hexenoate (4e): The reaction of $1 \mathrm{e}(174.5 \mathrm{mg}, 1.2 \mathrm{mmol}), \mathrm{K}_{2} \mathrm{CO}_{3}(14.0 \mathrm{mg}, 0.1 \mathrm{mmol})$ and diethyl malonate (163.4 $\mathrm{mg}, 1.0 \mathrm{mmol})$ in $2 \mathrm{ml}$ of acetone afforded $184.8 \mathrm{mg}(56 \%)$ of $4 \mathrm{e}$ : liquid; IR(neat) 3028, 2982, 1721, 1649, 1497, 1231, 1062, $700 \mathrm{~cm}^{-1} ;{ }^{1} \mathrm{H}$ NMR (300 MHz, $\left.\mathrm{CDCl}_{3}\right): \delta 7.12-7.27(\mathrm{~m}, 5 \mathrm{H})$, $4.63(\mathrm{t}, J=6.9 \mathrm{~Hz}, 1 \mathrm{H}), 4.10-4.27(\mathrm{~m}, 4 \mathrm{H}), 3.28(\mathrm{dd}, J=13.8$ and $7.5 \mathrm{~Hz}, 1 \mathrm{H}), 2.71(\mathrm{dd}, J=$ 13.8 and $6.6 \mathrm{~Hz}, 1 \mathrm{H}), 2.12(\mathrm{~s}, 3 \mathrm{H}), 1.94(\mathrm{~s}, 3 \mathrm{H}), 1.28(\mathrm{t}, J=7.2 \mathrm{~Hz}, 3 \mathrm{H}), 1.24(\mathrm{t}, J=6.9 \mathrm{~Hz}$, $3 \mathrm{H}) ;{ }^{13} \mathrm{C}$ NMR $\left(75 \mathrm{MHz}, \mathrm{CDCl}_{3}\right): \delta 205.5,164.8,152.1,138.6,128.7,128.1,127.8,126.2$, 61.1, 61.0, 58.7, 34.4, 29.5, 16.6, 13.8, 13.8; MS m/z (\%) $333\left(\mathrm{M}^{+}, 0.97\right), 198$ (100); HRMS $m / z$ (EI) calcd for $\mathrm{C}_{19} \mathrm{H}_{24} \mathrm{O}_{5} 332.16238$. Found 322.16389.

Synthesis of ethyl 2-(ethoxycarbonyl)-3-methyl-4-pentyl-5-oxo-2-tridecenoate (4f): The reaction of $\mathbf{1 f}(290.1 \mathrm{mg}, 1.2 \mathrm{mmol}), \mathrm{K}_{2} \mathrm{CO}_{3}(14.0 \mathrm{mg}, 0.1 \mathrm{mmol})$ and diethyl malonate (159.7 $\mathrm{mg}, 1.0 \mathrm{mmol}$ ) in $2 \mathrm{ml}$ of acetone afforded $349.0 \mathrm{mg}(85 \%)$ of $\mathbf{4 f}$ : liquid; IR(neat) 1723, 1627, 1224, 1058, 869, $724 \mathrm{~cm}^{-1} ;{ }^{1} \mathrm{H}$ NMR (300 MHz, $\left.\mathrm{CDCl}_{3}\right): \delta 4.01-4.22(\mathrm{~m}, 5 \mathrm{H}), 2.35(\mathrm{t}, J=7.4$ $\mathrm{Hz}, 2 \mathrm{H}), 1.76$ (s, $3 \mathrm{H}), 1.64-1.74(\mathrm{~m}, 1 \mathrm{H}), 1.35-1.41$ (m, $3 \mathrm{H}), 1.04-1.22$ (m, $22 \mathrm{H}), 0.68-0.80$ $(\mathrm{m}, 6 \mathrm{H}) ;{ }^{13} \mathrm{C}$ NMR $\left(75 \mathrm{MHz}, \mathrm{CDCl}_{3}\right): \delta 209.1,165.7,165.4,154.2,127.7,61.4,61.3,56.8$, $42.4,32.0,31.9,29.6,29.3,29.3,28.7,27.1,23.7,22.8,22.6,16.5,14.27,14.25,14.23,14.2$; MS $m / z(\%) 411\left(\mathrm{M}^{+}+1,5.93\right), 181$ (100); HRMS $m / z$ (EI) calcd for $\mathrm{C}_{24} \mathrm{H}_{42} \mathrm{O}_{5} 410.30323$. Found 410.30217. 
Synthesis of 4-(1', 1'-bis(ethoxycarbonyl)methyl)-5-methyl-4-hexen-2-one (3g): The reaction of $1 \mathrm{~g}(66.2 \mathrm{mg}, 0.65 \mathrm{mmol}), \mathrm{K}_{2} \mathrm{CO}_{3}(14.0 \mathrm{mg}, 0.1 \mathrm{mmol})$ and diethyl malonate $(80.7$ $\mathrm{mg}, 0.5 \mathrm{mmol})$ in $2 \mathrm{ml}$ of acetone afforded $107.5 \mathrm{mg}(75 \%)$ of $\mathbf{3 g}$ : liquid; IR(neat) 1731, 1307, 1222, 1158, $1025 \mathrm{~cm}^{-1} ;{ }^{1} \mathrm{H}$ NMR (300 MHz, $\mathrm{CDCl}_{3}$ ): $\delta 4.49$ (s, $\left.1 \mathrm{H}\right), 3.96-4.18(\mathrm{~m}, 4 \mathrm{H}), 3.28$ (s, $2 \mathrm{H}), 2.08$ (s, $3 \mathrm{H}), 1.77$ (s, $3 \mathrm{H}), 1.60$ (s, $3 \mathrm{H}), 1.19$ (t, J = 7.3 Hz, $6 \mathrm{H}) ;{ }^{13} \mathrm{C}$ NMR $(75 \mathrm{MHz}$, $\left.\mathrm{CDCl}_{3}\right): \delta 205.5,168.5,168.5,137.2,118.3,61.5,54.1,44.9,29.4,21.8,20.9,14.0 ; \mathrm{MS} m / z(\%)$ $270\left(\mathrm{M}^{+}, 1.35\right), 43(100)$; HRMS $m / z$ (EI) calcd for $\mathrm{C}_{14} \mathrm{H}_{22} \mathrm{O}_{5}$ 270.14673. Found 270.14875.

Synthesis of 2-methyl-3-(1', 1'-bis(ethoxycarbonyl)methyl)-2-undecen-5-one (3h): The reaction of $\mathbf{1 h}(117.3 \mathrm{mg}, 0.65 \mathrm{mmol}), \mathrm{K}_{2} \mathrm{CO}_{3}(14.3 \mathrm{mg}, 0.1 \mathrm{mmol})$ and diethyl malonate $(79.6$ $\mathrm{mg}, 0.5 \mathrm{mmol})$ in $2 \mathrm{ml}$ of acetone afforded $98.0 \mathrm{mg}$ (58\%) of $\mathbf{3 h}$ : liquid; IR(neat) 1731, 1733, 1307, 1146, 1036, $864 \mathrm{~cm}^{-1} ;{ }^{1} \mathrm{H}$ NMR (300 MHz, $\left.\mathrm{CDCl}_{3}\right): \delta 4.53(\mathrm{~s}, 1 \mathrm{H}), 4.05-4.23(\mathrm{~m}, 4 \mathrm{H})$, 3.39 (s, $2 \mathrm{H}), 2.39$ (t, $J=7.3 \mathrm{~Hz}, 2 \mathrm{H}), 1.81(\mathrm{~s}, 3 \mathrm{H}), 1.62(\mathrm{~s}, 3 \mathrm{H}), 1.47-1.60$ (m, $2 \mathrm{H}), 1.12-$ $1.40(\mathrm{~m}, 12 \mathrm{H}), 0.78-0.95(\mathrm{~m}, 3 \mathrm{H}) ;{ }^{13} \mathrm{C} \mathrm{NMR}\left(75 \mathrm{MHz}, \mathrm{CDCl}_{3}\right): \delta 207.8,168.8,137.2,118.5$, $61.7,54.4,44.3,42.5,31.9,29.2,24.1,22.7,22.1,21.1,14.3,14.2 ; \mathrm{MS} m / z(\%) 340\left(\mathrm{M}^{+}, 0.79\right)$, 43 (100); HRMS m/z (EI) calcd for $\mathrm{C}_{19} \mathrm{H}_{32} \mathrm{O}_{5} 340.22498$. Found 340.22777.

Synthesis of $(E)-4-\left(1^{\prime}, 1^{\prime}\right.$-bis(ethoxycarbonyl)methyl)-4-dodecen-2-one (E-3i): The reaction of $1 \mathbf{i}(216.3 \mathrm{mg}, 1.2 \mathrm{mmol}), \mathrm{K}_{2} \mathrm{CO}_{3}(13.4 \mathrm{mg}, 0.1 \mathrm{mmol})$ and diethyl malonate (166.1 $\mathrm{mg}, 1.0$ $\mathrm{mmol})$ in $2 \mathrm{ml}$ of acetone afforded $255.2 \mathrm{mg}(75 \%)$ of $E-3 \mathbf{3}$ : liquid; IR(neat) 1733, 1307, 1158, $1035 \mathrm{~cm}^{-1} ;{ }^{1} \mathrm{H} \mathrm{NMR}\left(300 \mathrm{MHz}, \mathrm{CDCl}_{3}\right): \delta 5.67(\mathrm{t}, J=7.2 \mathrm{~Hz}, 1 \mathrm{H}), 4.15(\mathrm{q}, J=7.2 \mathrm{~Hz}, 4 \mathrm{H})$, 4.04 (s, $1 \mathrm{H}), 3.39$ (s, $2 \mathrm{H}), 2.16$ (s, $3 \mathrm{H}), 1.98$ (q, $J=7.2 \mathrm{~Hz}, 2 \mathrm{H}), 1.18-1.40$ (m, $16 \mathrm{H}), 0.86$ (t, $J=6.6 \mathrm{~Hz}, 3 \mathrm{H}) ;{ }^{13} \mathrm{C} \mathrm{NMR}\left(75 \mathrm{MHz}, \mathrm{CDCl}_{3}\right): \delta 205.4,168.5,137.6,124.9,61.8,59.8,44.1$, 32.0, 29.8, 29.5, 29.4, 29.2, 29.0, 22.9, 14.3, 14.2; MS m/z (\%) $341\left(\mathrm{M}^{+}+1,20.39\right), 143$ (100); 
HRMS $m / z$ (EI) calcd for $\mathrm{C}_{19} \mathrm{H}_{32} \mathrm{O}_{5} 340.22498$. Found 340.22133. The stereochemistry of $E-3 \mathbf{i}$ was confirmed by the ${ }^{1} \mathrm{H}-{ }^{1} \mathrm{H}$ NOESY spectra.

Synthesis of $(E)-4-(1 ', 1$ '-bis(ethoxycarbonyl)methyl)-4-nonen-2-one (E-3j): The reaction of 1j (165.8 mg, $1.2 \mathrm{mmol}), \mathrm{K}_{2} \mathrm{CO}_{3}(14.4 \mathrm{mg}, 0.1 \mathrm{mmol})$ and diethyl malonate $(161.4 \mathrm{mg}, 1.0$ $\mathrm{mmol})$ in $2 \mathrm{ml}$ of acetone afforded $241.5 \mathrm{mg}(81 \%)$ of $E-\mathbf{3 j}$ : liquid; IR(neat) 1733, 1308, 1159, 1034, $866 \mathrm{~cm}^{-1} ;{ }^{1} \mathrm{H} \mathrm{NMR}\left(300 \mathrm{MHz}, \mathrm{CDCl}_{3}\right): \delta 5.63(\mathrm{t}, J=7.2 \mathrm{~Hz}, 1 \mathrm{H}), 4.10(\mathrm{q}, J=7.2 \mathrm{~Hz}$, $4 \mathrm{H}), 4.00$ (s, $1 \mathrm{H}), 3.36$ (s, $2 \mathrm{H}), 2.11$ (s, $3 \mathrm{H}), 1.89-2.00$ (m, $2 \mathrm{H}), 1.16-1.40$ (m, $10 \mathrm{H}), 0.83$ (t, $J=7.5 \mathrm{~Hz}, 3 \mathrm{H}) ;{ }^{13} \mathrm{C} \mathrm{NMR}\left(75 \mathrm{MHz}, \mathrm{CDCl}_{3}\right): \delta 205.3,168.4,137.5,124.9,61.8,59.8,44.0$, 31.3, 29.8, 28.6, 22.5, 14.2, 14.1; MS m/z (\%) $298\left(\mathrm{M}^{+}, 20.39\right), 139$ (100); HRMS m/z (EI) calcd for $\mathrm{C}_{16} \mathrm{H}_{26} \mathrm{O}_{5}$ 298.17802. Found 298.17864.

Synthesis of $(E)-10-(1 '$, 1'-bis(ethoxycarbonyl)methyl)-10-octadecen-8-one (E-3k): The reaction of $1 \mathrm{k}(316.8 \mathrm{mg}, 1.2 \mathrm{mmol}), \mathrm{K}_{2} \mathrm{CO}_{3}(14.1 \mathrm{mg}, 0.1 \mathrm{mmol})$ and diethyl malonate (162.7 $\mathrm{mg}, 1.0 \mathrm{mmol}$ ) in $2 \mathrm{ml}$ of acetone afforded $245.9 \mathrm{mg}(58 \%)$ of $E$-3k: liquid; IR(neat) 1736 , 1307, 1146, 1034, $723 \mathrm{~cm}^{-1} ;{ }^{1} \mathrm{H}$ NMR $\left(300 \mathrm{MHz}, \mathrm{CDCl}_{3}\right): \delta 5.66(\mathrm{t}, J=6.9 \mathrm{~Hz}, 1 \mathrm{H}), 4.14(\mathrm{q}$, $J=7.1 \mathrm{~Hz}, 4 \mathrm{H}), 4.04(\mathrm{~s}, 1 \mathrm{H}), 3.36(\mathrm{~s}, 2 \mathrm{H}), 2.42(\mathrm{t}, J=7.35 \mathrm{~Hz}, 2 \mathrm{H}), 1.96(\mathrm{q}, J=7.2 \mathrm{~Hz}, 2$ $\mathrm{H}), 1.54(\mathrm{q}, J=7.2 \mathrm{~Hz}, 2 \mathrm{H}), 1.11-1.41(\mathrm{~m}, 24 \mathrm{H}), 0.86(\mathrm{t}, J=6.6 \mathrm{~Hz}, 6 \mathrm{H}) ;{ }^{13} \mathrm{C} \mathrm{NMR}(75$ $\left.\mathrm{MHz}, \mathrm{CDCl}_{3}\right): \delta 207.4,168.5,168.5,137.2,124.9,61.7,59.8,43.2,42.6,32.0,31.9,29.47$, $29.41,29.39,29.35,29.3,29.2,28.9,24.02,22.85,22.82,14.30,14.29,14.2 ; \mathrm{MS} \mathrm{m} / \mathrm{z}(\%) 424$ $\left(\mathrm{M}^{+}, 20.39\right), 57$ (100); HRMS m/z (EI) calcd for $\mathrm{C}_{25} \mathrm{H}_{44} \mathrm{O}_{5}$ 424.31887. Found 424.32248.

Synthesis of (E)-3-butyl-4-(1', 1'-bis(ethoxycarbonyl)methyl)-4-decen-2-one (E-31): The reaction of 11 (226.4 mg, $1.2 \mathrm{mmol}), \mathrm{K}_{2} \mathrm{CO}_{3}(17.0 \mathrm{mg}, 0.1 \mathrm{mmol})$ and diethyl malonate (164.7 $\mathrm{mg}, 1.0 \mathrm{mmol}$ ) in $2 \mathrm{ml}$ of acetone afforded $310.2 \mathrm{mg}$ (84\%) of $E-31$ : liquid; IR(neat) 1755, 
1736, 1713, 1466, 1309, 1146, $1037 \mathrm{~cm}^{-1} ;{ }^{1} \mathrm{H}$ NMR (300 MHz, $\left.\mathrm{CDCl}_{3}\right): \delta 5.81(\mathrm{t}, J=7.2 \mathrm{~Hz}, 1$ H), 4.02-4.20 (m, $4 \mathrm{H}), 3.93(\mathrm{~s}, 1 \mathrm{H}), 3.64(\mathrm{dd}, J=8.4$ and $6.3 \mathrm{~Hz}, 1 \mathrm{H}), 2.22(\mathrm{q}, J=7.4 \mathrm{~Hz}, 2$ $\mathrm{H}), 2.10(\mathrm{~s}, 3 \mathrm{H}), 1.62-1.79(\mathrm{~m}, 1 \mathrm{H}), 1.02-1.50(\mathrm{~m}, 17 \mathrm{H}), 0.80-0.96(\mathrm{~m}, 6 \mathrm{H}) ;{ }^{13} \mathrm{C} \mathrm{NMR}(75$ $\left.\mathrm{MHz}, \mathrm{CDCl}_{3}\right): \delta 208.6,168.4,168.4,136.3,128.1,61.4,61.4,54.3,52.4,31.4,29.3,29.1,28.8$, 28.2, 27.7, 22.6, 22.4, 13.88, 13.85, 13.80, 13.79; MS m/z (\%) $368\left(\mathrm{M}^{+}, 1.90\right), 43$ (100); HRMS $m / z$ (EI) calcd for $\mathrm{C}_{21} \mathrm{H}_{36} \mathrm{O}_{5}$ 368.25628. Found 368.25509.

Synthesis of (E)-9-butyl-10-(1', 1'-bis(ethoxycarbonyl)methyl)-10-hexadecen-8-one (E3m): The reaction of $1 \mathrm{~m}(316.8 \mathrm{mg}, 1.2 \mathrm{mmol}), \mathrm{K}_{2} \mathrm{CO}_{3}(14.2 \mathrm{mg}, 0.1 \mathrm{mmol})$ and diethyl malonate $(161.7 \mathrm{mg}, 1.0 \mathrm{mmol})$ in $2 \mathrm{ml}$ of acetone afforded $321.4 \mathrm{mg}(71 \%)$ of E-3m: liquid; IR(neat) 1756, 1736, 1712, 1466, 1309, 1145, 1037, 876, $729 \mathrm{~cm}^{-1} ;{ }^{1} \mathrm{H}$ NMR $(300 \mathrm{MHz}$, $\left.\mathrm{CDCl}_{3}\right): \delta 5.75(\mathrm{t}, J=7.2 \mathrm{~Hz}, 1 \mathrm{H}), 4.02-4.19(\mathrm{~m}, 4 \mathrm{H}), 3.98(\mathrm{~s}, 1 \mathrm{H}), 3.64(\mathrm{t}, J=7.2 \mathrm{~Hz}, 1 \mathrm{H})$, 2.41-2.48 (m, $1 \mathrm{H}), 2.24-2.37(\mathrm{~m}, 1 \mathrm{H}), 2.22-2.27(\mathrm{q}, J=6.9 \mathrm{~Hz}, 2 \mathrm{H}), 1.61-1.78(\mathrm{~m}, 1 \mathrm{H})$, 1.01-1.59 (m, $27 \mathrm{H}), 0.78-0.92(\mathrm{~m}, 9 \mathrm{H}) ;{ }^{13} \mathrm{C} \mathrm{NMR}\left(75 \mathrm{MHz}, \mathrm{CDCl}_{3}\right): \delta 210.9,168.6,168.5$, $135.9,128.5,61.4,53.3,52.3,42.1,31.6,31.5,29.3,29.1,29.0,28.9,28.2,28.1,23.5,22.6$, 22.5, 22.4, 13.98, 13.92, 13.87, 13.86, 13.83; MS m/z (\%) $452\left(\mathrm{M}^{+}, 0.61\right), 57$ (100); HRMS m/z (EI) calcd for $\mathrm{C}_{27} \mathrm{H}_{48} \mathrm{O}_{5} 452.35018$. Found 452.35163 .

Synthesis of (E)-2-butyl-3-(1', 1'-bis(ethoxycarbonyl)methyl)-1-phenyl-3-nonen-1-one ( $E$ 3n): The reaction of $1 \mathrm{n}(324.5 \mathrm{mg}, 1.2 \mathrm{mmol}), \mathrm{K}_{2} \mathrm{CO}_{3}(17.2 \mathrm{mg}, 0.1 \mathrm{mmol})$ and diethyl malonate (164.1 mg, $1.0 \mathrm{mmol})$ in $2 \mathrm{ml}$ of acetone afforded $327.3 \mathrm{mg}$ (76\%) of $E$-3n: liquid; IR(neat) $1757,1734,1681,1597,1581,1306,1145,1038,717,691 \mathrm{~cm}^{-1} ;{ }^{1} \mathrm{H}$ NMR $(300 \mathrm{MHz}$, $\left.\mathrm{CDCl}_{3}\right): \delta 7.87(\mathrm{~d}, J=8.4 \mathrm{~Hz}, 2 \mathrm{H}), 7.47-7.55(\mathrm{~m}, 1 \mathrm{H}), 7.32-7.45(\mathrm{~m}, 2 \mathrm{H}), 5.77(\mathrm{t}, J=7.2 \mathrm{~Hz}$, $1 \mathrm{H}), 4.54(\mathrm{t}, J=7.05 \mathrm{~Hz}, 1 \mathrm{H}), 4.10-4.23(\mathrm{~m}, 3 \mathrm{H}), 3.79-3.98(\mathrm{~m}, 2 \mathrm{H}), 2.26-2.38(\mathrm{~m}, 2 \mathrm{H})$, 
1.79-1.96 (m, $1 \mathrm{H}), 1.49-1.61(\mathrm{~m}, 1 \mathrm{H}), 1.38-1.48$ (m, $2 \mathrm{H}), 1.11-1.47$ (m, $11 \mathrm{H}), 0.83-0.94$ (m, $9 \mathrm{H}) ;{ }^{13} \mathrm{C} \mathrm{NMR}\left(75 \mathrm{MHz}, \mathrm{CDCl}_{3}\right): \delta 201.4,169.0,168.6,137.7,137.7,135.6,133.1,128.8$, $128.6,128.4,61.7,61.4,52.2,49.1,31.9,29.9,29.6,29.0,28.8,23.0,22.8,14.3,14.2,14.2$, 13.8; MS m/z (\%) $430\left(\mathrm{M}^{+}, 0.69\right), 105$ (100); HRMS m/z (EI) calcd for $\mathrm{C}_{26} \mathrm{H}_{38} \mathrm{O}_{5} 430.27913$. Found 430.27524 .

Synthesis of (E)-2-butyl-3-(1', 1'-bis(ethoxycarbonyl)methyl)-1-(p-toyl)-3-nonen-1-one ( $E$ 3o): The reaction of $10(311.0 \mathrm{mg}, 1.2 \mathrm{mmol}), \mathrm{K}_{2} \mathrm{CO}_{3}(15.9 \mathrm{mg}, 0.1 \mathrm{mmol})$ and diethyl malonate (165.3 mg, $1.0 \mathrm{mmol})$ in $2 \mathrm{ml}$ of acetone afforded $208.5 \mathrm{mg}(47 \%)$ of $E$-3o: liquid; IR(neat) 2957, 1757, 1734, 1677, 1607, 1573, 936, 852, $820 \mathrm{~cm}^{-1} ;{ }^{1} \mathrm{H}$ NMR $(300 \mathrm{MHz}$, $\left.\mathrm{CDCl}_{3}\right): \delta 7.78(\mathrm{~d}, J=8.4 \mathrm{~Hz}, 2 \mathrm{H}), 7.16(\mathrm{~d}, J=8.4 \mathrm{~Hz}, 2 \mathrm{H}), 5.76(\mathrm{t}, J=7.2 \mathrm{~Hz}, 1 \mathrm{H}), 4.51(\mathrm{t}$, $J=7.2 \mathrm{~Hz}, 1 \mathrm{H}), 4.21(\mathrm{~s}, 1 \mathrm{H}), 4.16(\mathrm{q}, J=3.1 \mathrm{~Hz}, 2 \mathrm{H}), 3.86(\mathrm{q}, J=2.8 \mathrm{~Hz}, 2 \mathrm{H}), 2.36(\mathrm{~s}, 3$ H), 2.32 (q, $J=7.2 \mathrm{~Hz}, 2 \mathrm{H}), 1.80-1.95(\mathrm{~m}, 1 \mathrm{H}), 1.48-1.63(\mathrm{~m}, 1 \mathrm{H}) 1.41-4.49$ (m, $2 \mathrm{H}), 1.20-$ $1.40(\mathrm{~m}, 12 \mathrm{H}), 0.82-0.96(\mathrm{~m}, 8 \mathrm{H}) ;{ }^{13} \mathrm{C} \mathrm{NMR}\left(75 \mathrm{MHz}, \mathrm{CDCl}_{3}\right): \delta 200.6,169.0,168.3,143.7$, $135.1,134.9,129.0,128.7,128.2,61.3,61.1,51.90,48.5,31.9,29.7,29.3,28.8,28.5,22.7$, 22.5, 21.5, 13.98, 13.92, 13.91, 13.49; MS m/z (\%) $444\left(\mathrm{M}^{+}, 0.81\right), 119$ (100); HRMS m/z (EI) calcd for $\mathrm{C}_{27} \mathrm{H}_{40} \mathrm{O}_{5} 444.28758$. Found 444.28542. The stereochemistry of $E$-3o was confirmed by the ${ }^{1} \mathrm{H}-{ }^{1} \mathrm{H}$ NOESY spectra.

Synthesis of $(E)-3-p e n t y l-4-(1,1$ '-bis(ethoxycarbonyl)methyl)-4-decen-2-one ( $E$-3p): The reaction of $1 \mathbf{p}(266.8 \mathrm{mg}, 1.2 \mathrm{mmol}), \mathrm{K}_{2} \mathrm{CO}_{3}(16.4 \mathrm{mg}, 0.1 \mathrm{mmol})$ and diethyl malonate $(162.7$ $\mathrm{mg}, 1.0 \mathrm{mmol}$ ) in $2 \mathrm{ml}$ of acetone afforded $318.9 \mathrm{mg}(83 \%)$ of $E$-3p: liquid; IR(neat) 1755 , $1736,1713,1466,1146,1146,1037,871,729,630 \mathrm{~cm}^{-1} ; \quad{ }^{1} \mathrm{H}$ NMR $\left(300 \mathrm{MHz}, \mathrm{CDCl}_{3}\right): \delta 5.76$ $(\mathrm{t}, J=7.2 \mathrm{~Hz}, 1 \mathrm{H}), 3.99-4.12(\mathrm{~m}, 4 \mathrm{H}), 3.87(\mathrm{~s}, 1 \mathrm{H}), 3.60(\mathrm{t}, J=7.05 \mathrm{~Hz}, 1 \mathrm{H}), 2.17$ (q, $J=$ 
$7.1 \mathrm{~Hz}, 2 \mathrm{H}), 2.05(\mathrm{~s}, 3 \mathrm{H}), 1.59-1.72(\mathrm{~m}, 1 \mathrm{H}), 1.00-1.49(\mathrm{~m}, 19 \mathrm{H}), 0.74-0.85(\mathrm{~m}, 6 \mathrm{H}) ;{ }^{13} \mathrm{C}$ NMR (75 MHz, $\left.\mathrm{CDCl}_{3}\right): \delta 208.8,168.64,168.60,136.6,128.4,61.63,61.61,54.5,52.7,32.0$, 31.6, 29.6, 29.1, 28.4, 28.3, 26.91, 22.6, 14.14, 14.12, 14.10, 14.05; MS m/z (\%) $383\left(\mathrm{M}^{+}+1\right.$, 1.90), 43 (100); HRMS m/z (EI) calcd for $\mathrm{C}_{22} \mathrm{H}_{38} \mathrm{O}_{5} 382.27193$. Found 382.26824.

Synthesis of (E)-9-pentyl-10-(1', 1'-bis(ethoxycarbonyl)methyl)-10-hexadecen-8-one (E3q): The reaction of $\mathbf{1 q}(367.8 \mathrm{mg}, 1.2 \mathrm{mmol}), \mathrm{K}_{2} \mathrm{CO}_{3}(15.4 \mathrm{mg}, 0.1 \mathrm{mmol})$ and diethyl malonate $(163.2 \mathrm{mg}, 1.0 \mathrm{mmol})$ in $2 \mathrm{ml}$ of acetone afforded $364.0 \mathrm{mg}$ (78\%) of $E$-3q: liquid; IR(neat) 1756, 1736, 1712, 1466, 1309, 1145, 1037, 874, $727 \mathrm{~cm}^{-1} ;{ }^{1} \mathrm{H}$ NMR (300 MHz, $\left.\mathrm{CDCl}_{3}\right): \delta 5.74(\mathrm{t}, J=7.05 \mathrm{~Hz}, 1 \mathrm{H}), 4.01-4.15(\mathrm{~m}, 4 \mathrm{H}), 3.96(\mathrm{~s}, 1 \mathrm{H}), 3.64(\mathrm{t}, J=7.05 \mathrm{~Hz}, 1$ H), 2.41-2.52 (m, $1 \mathrm{H}), 2.16-2.23(\mathrm{~m}, 1 \mathrm{H}), 2.25-2.34(\mathrm{~m}, 2 \mathrm{H}), 1.63-1.75(\mathrm{~m}, 1 \mathrm{H}), 1.14-1.29$ (m, $29 \mathrm{H}), 0.78-0.85(\mathrm{~m}, 9 \mathrm{H}) ;{ }^{13} \mathrm{C} \mathrm{NMR}\left(75 \mathrm{MHz}, \mathrm{CDCl}_{3}\right): \delta 210.8,168.5,168.4,135.8,128.5$, $61.3,53.2,52.3,42.1,31.8,31.6,31.4,29.04,29.01,28.8,28.3,28.2,26.7,23.5,22.48,22.41$, 22.40, 13.92, 13.89, 13.88, 13.84, 13.82; MS m/z (\%) $466\left(\mathrm{M}^{+}, 1.46\right), 57$ (100); HRMS m/z (EI) calcd for $\mathrm{C}_{28} \mathrm{H}_{50} \mathrm{O}_{5} 466.36583$. Found 466.36641

Synthesis of (E)-2-pentyl-3-(1', 1'-bis(ethoxycarbonyl)methyl)-1-phenyl-3-nonen-1-one (E-3r): The reaction of $1 \mathbf{r}(341.3 \mathrm{mg}, 1.2 \mathrm{mmol}), \mathrm{K}_{2} \mathrm{CO}_{3}(16.4 \mathrm{mg}, 0.1 \mathrm{mmol})$ and diethyl malonate $(160.0 \mathrm{mg}, 1.0 \mathrm{mmol})$ in $2 \mathrm{ml}$ of acetone afforded $332.4 \mathrm{mg}(75 \%)$ of $E-3 \mathrm{r}$ : liquid; IR(neat) $1757,1735,1681,1597,1581,1448,1306,1145,1038,717,691 \mathrm{~cm}^{-1} ;{ }^{1} \mathrm{H}$ NMR $(300$ $\left.\mathrm{MHz}, \mathrm{CDCl}_{3}\right): \delta 7.85(\mathrm{~d}, J=7.5 \mathrm{~Hz}, 2 \mathrm{H}), 7.48(\mathrm{t}, J=7.2 \mathrm{~Hz}, 1 \mathrm{H}), 7.37(\mathrm{t}, J=5.0 \mathrm{~Hz}, 2 \mathrm{H})$, $5.75(\mathrm{t}, J=7.5 \mathrm{~Hz}, 1 \mathrm{H}), 4.52(\mathrm{t}, J=7.4 \mathrm{~Hz}, 1 \mathrm{H}), 4.10-4.18(\mathrm{~m}, 3 \mathrm{H}), 3.78-3.85(\mathrm{~m}, 2 \mathrm{H}), 2.30$ $(\mathrm{q}, J=7.5 \mathrm{~Hz}, 2 \mathrm{H}), 1.75-1.90(\mathrm{~m}, 1 \mathrm{H}), 1.18-1.58(\mathrm{~m}, 16 \mathrm{H}), 0.83-0.90(\mathrm{~m}, 9 \mathrm{H}) ;{ }^{13} \mathrm{C} \mathrm{NMR}$ $\left(75 \mathrm{MHz}, \mathrm{CDCl}_{3}\right): \delta 201.5,169.0,168.5,137.69,137.68,135.6,133.1,128.8,128.5,128.4$, 
$61.6,61.3,52.2,49.1,32.1,31.8,30.1,29.0,28.8,27.1,22.7,14.21,14.18,13.7 ; \mathrm{MS} \mathrm{m} / \mathrm{z}(\%)$

$429\left(\mathrm{M}^{+}-\mathrm{CH}_{3}, 0.12\right), 105$ (100); HRMS $\mathrm{m} / \mathrm{z}$ (EI) calcd for $\mathrm{C}_{27} \mathrm{H}_{40} \mathrm{O}_{5}$ 444.28758. Found 444.28764.

Synthesis of 2-(1', 1'-bis(ethoxycarbonyl)methyl)-1-octen-4-one (3s) and ethyl 2-

(ethoxycarbonyl)-3-methyl-5-oxo-2-nonenoate (4s): The reaction of 1s (149.0 mg, 1.2

$\mathrm{mmol}), \mathrm{K}_{2} \mathrm{CO}_{3}(13.8 \mathrm{mg}, 0.1 \mathrm{mmol})$ and diethyl malonate $(156.7 \mathrm{mg}, 1.0 \mathrm{mmol})$ in $2 \mathrm{ml}$ of acetone afforded $238.9 \mathrm{mg}(88 \%)$ of $\mathbf{3 s}$ and $\mathbf{4 s}$ as a mixture: liquid; IR(neat) 1732, 1644, 1597 , 1466, 1368, 1305, 1150, 1038, 918, $864 \mathrm{~cm}^{-1} ;{ }^{1} \mathrm{H}$ NMR (300 MHz, $\left.\mathrm{CDCl}_{3}\right)$ 3s: $\delta 5.28(\mathrm{~s}, 1 \mathrm{H})$, $5.16(\mathrm{~s}, 1 \mathrm{H}), 4.05-4.30(\mathrm{~m}, 5 \mathrm{H}), 3.33(\mathrm{~s}, 2 \mathrm{H}), 2.45(\mathrm{t}, J=7.5 \mathrm{~Hz}, 2 \mathrm{H}), 1.43-1.60(\mathrm{~m}, 2 \mathrm{H})$, 1.20-1.40 (m, $8 \mathrm{H}), 0.88(\mathrm{t}, J=7.1 \mathrm{~Hz}, 3 \mathrm{H})$. The following data are discernible for $4 \mathrm{~s}$ : $4.05-$ $4.30(\mathrm{~m}, 4 \mathrm{H}), 3.64$ (s, $2 \mathrm{H}), 2.45(\mathrm{t}, J=7.5 \mathrm{~Hz}, 2 \mathrm{H}), 2.00$ (s, $3 \mathrm{H}) ; \mathrm{MS} m / z(\%) 284\left(\mathrm{M}^{+}, 1.14\right)$, 85 (100); HRMS m/z (EI) calcd for $\mathrm{C}_{15} \mathrm{H}_{24} \mathrm{O}_{5}$ 284.16237. Found 284.16297.

Synthesis of 2-(1', 1'-bis(ethoxycarbonyl)methyl)-1-nonen-4-one (3t) and ethyl 2(ethoxycarbonyl)-3-methyl-5-oxo-2-decenoate (4t): The reaction of $\mathbf{1 t}(158.6 \mathrm{mg}, 1.2 \mathrm{mmol})$, $\mathrm{K}_{2} \mathrm{CO}_{3}(16.7 \mathrm{mg}, 0.1 \mathrm{mmol})$ and diethyl malonate $(163.7 \mathrm{mg}, 1.0 \mathrm{mmol})$ in $2 \mathrm{ml}$ of acetone afforded $251.6 \mathrm{mg}$ (89\%) of $\mathbf{3 t}$ and $\mathbf{4 t}$ as a mixture: liquid; IR(neat) 1735, 1644, 1467, 1368, 1310, 1148, 1037, 919, 864, $730 \mathrm{~cm}^{-1} ;{ }^{1} \mathrm{H}$ NMR (300 MHz, $\left.\mathrm{CDCl}_{3}\right) 3 \mathrm{t}: \delta 5.27(\mathrm{~s}, 1 \mathrm{H}), 5.15(\mathrm{~s}$, $1 \mathrm{H}), 4.10-4.25(\mathrm{~m}, 5 \mathrm{H}), 3.32(\mathrm{~s}, 2 \mathrm{H}), 2.44(\mathrm{t}, J=7.2 \mathrm{~Hz}, 2 \mathrm{H}), 1.50-1.60(\mathrm{~m}, 2 \mathrm{H}), 1.18-1.30$ $(\mathrm{m}, 10 \mathrm{H}), 0.85(\mathrm{t}, J=7.2 \mathrm{~Hz}, 3 \mathrm{H})$. The following data are discernible for $\mathbf{4 t}: 4.10-4.25(\mathrm{~m}, 4$ H), $3.62(\mathrm{~s}, 2 \mathrm{H}), 2.44$ (t, J=7.2 Hz, $2 \mathrm{H}), 1.99$ (s, $3 \mathrm{H})$; MS m/z (\%) 298 (M+, 1.936), 99 (100); HRMS calcd for $\mathrm{C}_{16} \mathrm{H}_{26} \mathrm{O}_{5}$ 298.17802. Found 298.17593.

Synthesis of 2-(1', 1'-bis(ethoxycarbonyl)methyl)-1-decen-4-one (3u) and ethyl 2- 
(ethoxycarbonyl)-3-methyl-5-oxo-2-undecenoate (4u): The reaction of $\mathbf{1 u}(173.1 \mathrm{mg}, 1.2$ $\mathrm{mmol}), \mathrm{K}_{2} \mathrm{CO}_{3}(14.7 \mathrm{mg}, 0.1 \mathrm{mmol})$ and diethyl malonate $(162.4 \mathrm{mg}, 1.0 \mathrm{mmol})$ in $2 \mathrm{ml}$ of acetone afforded $267.6 \mathrm{mg}(86 \%)$ of $\mathbf{3 u}$ and $\mathbf{4} \mathbf{u}$ as a mixture: liquid; IR(neat) 1735, 1644, 1467, 1368, 1306, 1150, 1037, 918, 965, 786, $727 \mathrm{~cm}^{-1} ;{ }^{1} \mathrm{H}$ NMR (300 MHz, $\left.\mathrm{CDCl}_{3}\right)$ 3u: $\delta 5.15(\mathrm{~s}, 1$ H), $5.04(\mathrm{~s}, 1 \mathrm{H}), 4.01-4.20(\mathrm{~m}, 5 \mathrm{H}), 3.21(\mathrm{~s}, 2 \mathrm{H}), 2.33(\mathrm{t}, J=7.8 \mathrm{~Hz}, 2 \mathrm{H}), 1.39-1.51(\mathrm{~m}, 2 \mathrm{H})$, 1.08-1.25 (m, $12 \mathrm{H}), 0.73(\mathrm{t}, J=8.4 \mathrm{~Hz}, 3 \mathrm{H})$. The following data are discernible for $4 \mathbf{u}: 4.01$ $4.20(\mathrm{~m}, 4 \mathrm{H}), 3.51(\mathrm{~s}, 2 \mathrm{H}), 2.33$ (t, $J=7.8 \mathrm{~Hz}, 2 \mathrm{H}), 1.87$ (s, $3 \mathrm{H}) ; \mathrm{MS} m / z(\%) 312\left(\mathrm{M}^{+}, 1.14\right)$, 43 (100); HRMS m/z (EI) calcd for $\mathrm{C}_{17} \mathrm{H}_{28} \mathrm{O}_{5} 312.19367$. Found 312.19365.

Synthesis of 2-(1', 1'-bis(ethoxycarbonyl)methyl)-1-dodecen-4-one (3v) and ethyl 2(ethoxycarbonyl)-3-methyl-5-oxo-2-tridecenoate (4v): The reaction of $\mathbf{1 v}(208.1 \mathrm{mg}, 1.2$ $\mathrm{mmol}), \mathrm{K}_{2} \mathrm{CO}_{3}(15.7 \mathrm{mg}, 0.1 \mathrm{mmol})$ and diethyl malonate $(163.3 \mathrm{mg}, 1.0 \mathrm{mmol})$ in $2 \mathrm{ml}$ of acetone afforded $240.0 \mathrm{mg}(74 \%)$ of $\mathbf{3 v}$ and $\mathbf{4 v}$ as a mixture: liquid; IR(neat) 1733, 1644, 1597, 1466, 1368, 1309, 1148, 1037, 917, 864, $723 \mathrm{~cm}^{-1} ;{ }^{1} \mathrm{H}$ NMR (300 MHz, $\left.\mathrm{CDCl}_{3}\right)$ 3v : $\delta 5.19(\mathrm{~s}$, $1 \mathrm{H}), 5.07(\mathrm{~s}, 1 \mathrm{H}), 4.04-4.11(\mathrm{~m}, 5 \mathrm{H}), 3.23(\mathrm{~s}, 2 \mathrm{H}), 2.36(\mathrm{t}, J=7.5 \mathrm{~Hz}, 2 \mathrm{H}), 1.40-1.50(\mathrm{~m}, 2$ $\mathrm{H}), 1.07-1.22(\mathrm{~m}, 16 \mathrm{H}), 0.76(\mathrm{t}, J=6.6 \mathrm{~Hz}, 3 \mathrm{H})$. The following data are discernible for $4 \mathbf{v}$ : 4.04-4.11 (m, $4 \mathrm{H}), 3.53$ (s, $2 \mathrm{H}), 2.36$ (t, $J=7.5 \mathrm{~Hz}, 2 \mathrm{H}), 1.90$ (s, $3 \mathrm{H})$; MS m/z (\%) $340\left(\mathrm{M}^{+}\right.$, 1.14), 57 (100); HRMS m/z (EI) calcd for $\mathrm{C}_{19} \mathrm{H}_{32} \mathrm{O}_{5} 340.22497$. Found 340.22378.

\section{Synthesis of $\alpha$-Pyrone.}

Synthesis of 3-(ethoxycarbonyl)-4, 6-dimethyl-5-butyl-2-pyranone (6a): The reaction of 1a (166 mg , $1.2 \mathrm{mmol}), \mathrm{K}_{2} \mathrm{CO}_{3}(14.0 \mathrm{mg}, 0.1 \mathrm{mmol})$ and diethyl malonate $(160 \mathrm{mg}, 1 \mathrm{mmol})$ in 2 $\mathrm{ml}$ of acetone afforded $161 \mathrm{mg}(64 \%)$ of $\mathbf{6 a}$.

Synthesis of 3-(ethoxycarbonyl)-4, 6-dimethyl-5-ethyl-2-pyranone (6b): The reaction of $\mathbf{1 b}$ 
(132 mg , $1.2 \mathrm{mmol}), \mathrm{K}_{2} \mathrm{CO}_{3}(14.0 \mathrm{mg}, 0.1 \mathrm{mmol})$ and diethyl malonate $(160 \mathrm{mg}, 1 \mathrm{mmol})$ in 2 $\mathrm{ml}$ of ethanol afforded $118 \mathrm{mg}(53 \%)$ of $\mathbf{6 b}$ : yellow oil, ${ }^{1} \mathrm{HNMR}\left(300 \mathrm{MHz}, \mathrm{CDCl}_{3}\right) \delta 4.34(\mathrm{q}$, $J=7.0 \mathrm{~Hz}, 2 \mathrm{H}), 2.37(\mathrm{t}, J=7.5 \mathrm{~Hz}, 2 \mathrm{H}), 2.24(\mathrm{~s}, 3 \mathrm{H}), 2.16(\mathrm{~s}, 3 \mathrm{H}), 1.33(\mathrm{t}, J=7.1 \mathrm{~Hz}, 3 \mathrm{H})$, $1.04(\mathrm{t}, J=7.4 \mathrm{~Hz}, 3 \mathrm{H})$; MS m/z (\%) $224\left(\mathrm{M}^{+}, 4\right), 43(100)$; IR 1720, 1635, 1552, $1236 \mathrm{~cm}^{-1}$; HRMS calcd for $\mathrm{C}_{12} \mathrm{H}_{16} \mathrm{O}_{4}$ 224.1044. Found 224.1063.

Synthesis of 3-(ethoxycarbonyl)-4, 6-dimethyl-5-hexyl-2-pyranone (6c): The reaction of 1c (199.5 mg, $1.2 \mathrm{mmol}), \mathrm{K}_{2} \mathrm{CO}_{3}(15.2 \mathrm{mg}, 0.1 \mathrm{mmol})$ and diethyl malonate (160.0 mg, $\left.1.0 \mathrm{mmol}\right)$ in $2 \mathrm{ml}$ of acetone afforded $225.8 \mathrm{mg}(81 \%)$ of $\mathbf{6 c}$.

Synthesis of 3-(ethoxycarbonyl)-4, 6-dimethyl-5-allyl-2-pyranone (6d): The reaction of 1d (146 mg, $1.2 \mathrm{mmol}), \mathrm{K}_{2} \mathrm{CO}_{3}(14.0 \mathrm{mg}, 0.1 \mathrm{mmol})$ and diethyl malonate $(160 \mathrm{mg}, 1 \mathrm{mmol})$ in 2 $\mathrm{ml}$ of acetone afforded $213 \mathrm{mg}(90 \%)$ of $\mathbf{6 d}$ : yellow oil, ${ }^{1} \mathrm{H} \mathrm{NMR}\left(300 \mathrm{MHz}, \mathrm{CDCl}_{3}\right) \delta 5.88-$ $5.76(\mathrm{~m}, 1 \mathrm{H}), 5.12-5.08(\mathrm{~d}, J=10.3 \mathrm{~Hz}, 1 \mathrm{H}), 4.95(\mathrm{~d}, J=17.0 \mathrm{~Hz}, 1 \mathrm{H}), 4.36(\mathrm{q}, J=7.2 \mathrm{~Hz}, 2$ H), $3.10(\mathrm{t}, J=3.2 \mathrm{~Hz}, 2 \mathrm{H}), 2.24(\mathrm{~s}, 3 \mathrm{H}), 2.14(\mathrm{~s}, 3 \mathrm{H}), 1.35(\mathrm{t}, J=7.1 \mathrm{~Hz}, 3 \mathrm{H}) ; \mathrm{MS} \mathrm{m} / z(\%)$ $237\left(\mathrm{M}^{+}+1,8\right), 236\left(\mathrm{M}^{+}, 52\right), 208$ (100); IR 1739, 1716, 1639, 1554, $1238 \mathrm{~cm}^{-1}$; HRMS calcd for $\mathrm{C}_{13} \mathrm{H}_{16} \mathrm{O}_{4}$ 236.1044. Found 236.1030.

Synthesis of 3-(ethoxycarbonyl)-4, 6-dimethyl-5-benzyl-2-pyranone (6e): The reaction of 1e (192 mg, $1.2 \mathrm{mmol}), \mathrm{K}_{2} \mathrm{CO}_{3}(14.0 \mathrm{mg}, 0.1 \mathrm{mmol})$ and diethyl malonate (160 mg, $\left.1 \mathrm{mmol}\right)$ in $2 \mathrm{ml}$ of acetone afforded $262 \mathrm{mg}(92 \%)$ of $\mathbf{6 e}$ : white solid, recrystallized from $\mathrm{CH}_{2} \mathrm{Cl}_{2} /$ hexane (1:20), m.p.79.5-80.5 ${ }^{\circ} \mathrm{C} ;{ }^{1} \mathrm{H}$ NMR (300 MHz, $\left.\mathrm{CDCl}_{3}\right) \delta 7.34-7.23(\mathrm{~m}, 3 \mathrm{H}), 7.08$ (d, J=7.2 Hz, $2 \mathrm{H}), 4.36(\mathrm{q}, J=7.2 \mathrm{~Hz}, 2 \mathrm{H}), 3.77(\mathrm{~s}, 2 \mathrm{H}), 2.30(\mathrm{~s}, 3 \mathrm{H}), 2.04(\mathrm{~s}, 3 \mathrm{H}), 1.34$ (t, $J=7.1 \mathrm{~Hz}, 3$ $\mathrm{H}) ;{ }^{13} \mathrm{C} \mathrm{NMR}\left(125 \mathrm{MHz}, \mathrm{CDCl}_{3}\right) \delta 165.2,160.1,159.1,155.5,137.4,128.9,127.4,126.8$, 118.5, 114.1, 61.8, 32.4, 18.0, 17.5, 14.1; MS m/z (\%) 287( $\left.\mathrm{M}^{+}+1,14\right), 286\left(\mathrm{M}^{+}, 38\right), 218(100)$; 
IR 1726, 1699, 1557, $1236 \mathrm{~cm}^{-1}$; HRMS calcd for $\mathrm{C}_{17} \mathrm{H}_{18} \mathrm{O}_{4}$ 286.1200. Found 286.1204 Synthesis of 3-(ethoxycarbonyl)-4-methyl-5-pentyl-6-octyl-2-pyranone (6f): The reaction of 1f $(294.2 \mathrm{mg}, 1.2 \mathrm{mmol}), \mathrm{K}_{2} \mathrm{CO}_{3}(14.0 \mathrm{mg}, 0.1 \mathrm{mmol})$ and diethyl malonate $(160.4 \mathrm{mg}, 1.0$ $\mathrm{mmol}$ ) in $2 \mathrm{ml}$ of acetone afforded $204.4 \mathrm{mg}(56 \%)$ of $\mathbf{6 f}$ : liquid; IR(neat) 1723, 1631, 1551, 1466, 1237, 1032, 860, 786, $724 \mathrm{~cm}^{-1} ;{ }^{1} \mathrm{H}$ NMR (300 MHz, $\left.\mathrm{CDCl}_{3}\right): \delta 4.30(\mathrm{t}, J=7.2 \mathrm{~Hz}, 2 \mathrm{H})$, $2.44(\mathrm{t}, J=7.5 \mathrm{~Hz}, 2 \mathrm{H}), 2.26(\mathrm{t}, J=7.2 \mathrm{~Hz}, 2 \mathrm{H}), 2.11(\mathrm{~s}, 3 \mathrm{H}), 1.50-1.63(\mathrm{~m}, 2 \mathrm{H}), 1.18-1.40$ (m, $19 \mathrm{H}), 0.78-0.86(\mathrm{~m}, 6 \mathrm{H}) ;{ }^{13} \mathrm{C} \mathrm{NMR}\left(75 \mathrm{MHz}, \mathrm{CDCl}_{3}\right): \delta 165.7,162.5,159.6,155.2,118.5$, $116.2,61.9,32.0,31.4,30.1,29.5,29.4,29.3,27.9,26.8,22.8,22.6,17.4,14.3,14.24,14.22$, 14.1; MS m/z (\%) $364\left(\mathrm{M}^{+}, 12.93\right), 43$ (100); HRMS $m / z$ (EI) calcd for $\mathrm{C}_{22} \mathrm{H}_{36} \mathrm{O}_{4} 364.26136$. Found 364.26224.

Synthesis of 3-(ethoxycarbonyl)-4-pentyl-6-methyl -2-pyranone (6j): The reaction of $\mathbf{1 j}$ (166 mg, $1.2 \mathrm{mmol}), \mathrm{K}_{2} \mathrm{CO}_{3}(14.0 \mathrm{mg}, 0.1 \mathrm{mmol})$ and diethyl malonate $(160 \mathrm{mg}, 1 \mathrm{mmol})$ in 1 $\mathrm{ml}$ of DMF afforded $129 \mathrm{mg}(54 \%)$ of $\mathbf{6 j}$ : yellow oil, IR 1724, 1645, 1565, 1256, $1276 \mathrm{~cm}^{-1} ;{ }^{1} \mathrm{H}$ NMR (300 MHz, CDCl $)_{3} \delta 5.93(\mathrm{~s}, 1 \mathrm{H}), 4.35(\mathrm{q}, J=7.1 \mathrm{~Hz}, 2 \mathrm{H}), 2.44(\mathrm{t}, J=8.0 \mathrm{~Hz}, 2 \mathrm{H})$, 2.24 (s, $3 \mathrm{H}), 1.59$ (m, $3 \mathrm{H}), 1.37-1.30$ (m, $6 \mathrm{H}), 0.88$ (t, $J=6.7 \mathrm{~Hz}, 3 \mathrm{H}) ; \mathrm{MS} \mathrm{m} / z(\%) 253$ $\left(\mathrm{M}^{+}+1,7\right), 252\left(\mathrm{M}^{+}, 25\right), 163$ (100); HRMS calcd for $\mathrm{C}_{14} \mathrm{H}_{20} \mathrm{O}_{4}$ 252.1356. Found 252.1339. Synthesis of 3-(ethoxycarbonyl)-4-methyl-6-butyl-2-pyranone (6s): The reaction of 1s (141.4 mg, $1.2 \mathrm{mmol}), \mathrm{K}_{2} \mathrm{CO}_{3}(17.5 \mathrm{mg}, 0.1 \mathrm{mmol})$ and diethyl malonate $(160.1 \mathrm{mg}, 1.0 \mathrm{mmol})$ in $2 \mathrm{ml}$ of acetone afforded $155.8 \mathrm{mg}(65 \%)$ of $6 \mathrm{~s}$ : liquid; IR(neat) 1724, 1644, 1566, 1259, $1178,1076,1025,838,790 \mathrm{~cm}^{-1} ;{ }^{1} \mathrm{H}$ NMR $\left(300 \mathrm{MHz}, \mathrm{CDCl}_{3}\right): \delta 5.86(\mathrm{~s}, 1 \mathrm{H}), 4.29$ (q, J = 7.2 $\mathrm{Hz}, 2 \mathrm{H}), 2.40(\mathrm{t}, J=7.5 \mathrm{~Hz}, 2 \mathrm{H}), 2.16(\mathrm{~s}, 3 \mathrm{H}), 1.51-1.61(\mathrm{~m}, 2 \mathrm{H}), 1.22-1.32(\mathrm{~m}, 5 \mathrm{H}), 0.85$ $(\mathrm{t}, J=7.5 \mathrm{~Hz}, 3 \mathrm{H}) ;{ }^{13} \mathrm{C} \mathrm{NMR}\left(75 \mathrm{MHz}, \mathrm{CDCl}_{3}\right): \delta 166.7,165.1,160.0,156.8,116.2,106.8$, 
61.6, 33.5, 29.0, 22.2, 20.1, 14.3, 13.8; MS m/z (\%) $238\left(\mathrm{M}^{+}, 48.93\right), 153$ (100); HRMS m/z (EI) calcd for $\mathrm{C}_{13} \mathrm{H}_{18} \mathrm{O}_{4}$ 238.12051. Found 238.12478.

Synthesis of 3-(ethoxycarbonyl)-4-methyl-6-hexyl-2-pyranone (6u): The reaction of $1 u$ (177.5 mg, $1.2 \mathrm{mmol}), \mathrm{K}_{2} \mathrm{CO}_{3}(14.2 \mathrm{mg}, 0.1 \mathrm{mmol})$ and diethyl malonate (157.4 mg, $\left.1.0 \mathrm{mmol}\right)$ in $2 \mathrm{ml}$ of acetone afforded $167.8 \mathrm{mg}(63 \%)$ of $\mathbf{6 u}$ : liquid; IR(neat) 1726, 1644, 1566, 1259, 1177, 1076, 1024, 958, 839, $789 \mathrm{~cm}^{-1} ;{ }^{1} \mathrm{H}$ NMR (300 MHz, $\left.\mathrm{CDCl}_{3}\right): \delta 5.88(\mathrm{~s}, 1 \mathrm{H}), 4.35(\mathrm{q}, J$ $=7.2 \mathrm{~Hz}, 2 \mathrm{H}), 2.44(\mathrm{t}, J=7.6 \mathrm{~Hz}, 2 \mathrm{H}), 2.21(\mathrm{~s}, 3 \mathrm{H}), 1.58-1.67(\mathrm{~m}, 2 \mathrm{H}), 1.20-1.40(\mathrm{~m}, 9 \mathrm{H})$, $0.87(\mathrm{t}, J=6.0 \mathrm{~Hz}, 3 \mathrm{H}) ;{ }^{13} \mathrm{C} \mathrm{NMR}\left(75 \mathrm{MHz}, \mathrm{CDCl}_{3}\right): \delta 166.6,165.1,160.0,156.4,116.2$, 106.7, 61.8, 33.9, 31.5, 28.8, 26.9, 22.6, 20.4, 14.4, 14.2; MS m/z (\%) $266\left(\mathrm{M}^{+}, 25.93\right), 153$ (100); HRMS $m / z$ (EI) calcd for $\mathrm{C}_{15} \mathrm{H}_{22} \mathrm{O}_{4} \quad$ 266.15181. Found 266.14950.

Synthesis of 3-(ethoxycarbonyl)-4-methyl-6-octyl-2-pyranone (6v): The reaction of $1 \mathrm{v}$ (206.7 mg, $1.2 \mathrm{mmol}), \mathrm{K}_{2} \mathrm{CO}_{3}(15.8 \mathrm{mg}, 0.1 \mathrm{mmol})$ and diethyl malonate $(160.0 \mathrm{mg}, 1.0 \mathrm{mmol})$ in $2 \mathrm{ml}$ of acetone afforded $203.1 \mathrm{mg}(69 \%)$ of $\mathbf{6 v}$ : liquid; IR(neat) 1725, 1644, 1566, 1258, 1177, 1076, 1024, 966, 839, $789 \mathrm{~cm}^{-1} ;{ }^{1} \mathrm{H}$ NMR (300 MHz, $\left.\mathrm{CDCl}_{3}\right): \delta 5.88$ (s, $\left.1 \mathrm{H}\right), 4.34$ (q, $J$ $=7.5 \mathrm{~Hz}, 2 \mathrm{H}), 2.44(\mathrm{t}, J=7.5 \mathrm{~Hz}, 2 \mathrm{H}), 2.21(\mathrm{~s}, 3 \mathrm{H}), 1.55-1.65(\mathrm{~m}, 2 \mathrm{H}), 1.18-1.40(\mathrm{~m}, 13 \mathrm{H})$, $0.86(\mathrm{t}, J=6.6 \mathrm{~Hz}, 3 \mathrm{H}) ;{ }^{13} \mathrm{C} \mathrm{NMR}\left(75 \mathrm{MHz}, \mathrm{CDCl}_{3}\right): \delta 166.7,165.2,160.0,156.4,116.3$, 106.7, 61.9, 34.0, 32.0, 29.4, 29.3, 29.2, 27.0, 22.8, 20.4, 14.4, 14.3; MS m/z (\%) $294\left(\mathrm{M}^{+}\right.$, 11.49), 153 (100); HRMS m/z (EI) calcd for $\mathrm{C}_{17} \mathrm{H}_{26} \mathrm{O}_{4}$ 294.18311. Found 294.18072.

Synthesis of 3-(ethoxycarbonyl)-4, 6-dimethyl-2-pyranone (6w): The reaction of 1 w (98 mg, $1.2 \mathrm{mmol}), \mathrm{K}_{2} \mathrm{CO}_{3}(14.0 \mathrm{mg}, 0.1 \mathrm{mmol})$ and diethyl malonate $(160 \mathrm{mg}, 1 \mathrm{mmol})$ in $2 \mathrm{ml}$ of ethanol afforded $147 \mathrm{mg}(74 \%)$ of 6 w. m.p. $54-55^{\circ} \mathrm{C}$ IR 1740, 1693, 1643, 1552, 1253, 1191 $\mathrm{cm}^{-1} ;{ }^{1} \mathrm{H}$ NMR $\left(300 \mathrm{MHz}, \mathrm{CDCl}_{3}\right) \delta 5.82(\mathrm{~s}, 1 \mathrm{H}), 4.25(\mathrm{q}, J=7.2 \mathrm{~Hz}, 2 \mathrm{H}), 2.13(\mathrm{~s}, 3 \mathrm{H}), 2.11$ 
(s, $3 \mathrm{H}), 1.25(\mathrm{t}, J=7.1 \mathrm{~Hz}, 3 \mathrm{H}) ; \mathrm{MS} m / z(\%) 197\left(\mathrm{M}^{+}+1,13\right), 196\left(\mathrm{M}^{+}, 51\right), 151(100)$;

Elemental Analysis calcd for $\mathrm{C}_{10} \mathrm{H}_{12} \mathrm{O}_{4} \mathrm{C}$ 61.22, H 6.16. Found C 61.21, H 6.21 .

Synthesis of 3-(ethoxycarbonyl)-4-methyl-6-phenyl-2-pyranone (6x):The reaction of $1 \mathbf{x}$

(173 mg, $1.2 \mathrm{mmol}), \mathrm{K}_{2} \mathrm{CO}_{3}(14.0 \mathrm{mg}, 0.1 \mathrm{mmol})$ and diethyl malonate $(160 \mathrm{mg}, 1 \mathrm{mmol})$ in 2 $\mathrm{ml}$ of acetone afforded $184 \mathrm{mg}(71 \%)$ of $\mathbf{6 x}$ : white solid, recrystallized from

$\mathrm{CH}_{2} \mathrm{Cl}_{2} /$ hexane(1:20), m.p.57.0-57.5 ${ }^{\circ} \mathrm{C}$; IR 1744, 1722, 1553, $1266 \mathrm{~cm}^{-1} ;{ }^{1} \mathrm{H}$ NMR(300 MHz, $\left.\mathrm{CDCl}_{3}\right) \delta 7.84-7.81(\mathrm{~m}, 2 \mathrm{H}), 7.47-7.45(\mathrm{~m}, 3 \mathrm{H}), 6.57(\mathrm{~s}, 1 \mathrm{H}), 4.40(\mathrm{q}, J=7.2 \mathrm{~Hz}, 2 \mathrm{H}), 2.34$ (s, $3 \mathrm{H}), 1.38(\mathrm{t}, J=7.1 \mathrm{~Hz}, 3 \mathrm{H})$; MS m/z (\%) $259\left(\mathrm{M}^{+}+1,8\right), 258\left(\mathrm{M}^{+}, 62\right), 230(100)$;

Elemental Analysis calcd for $\mathrm{C}_{15} \mathrm{H}_{14} \mathrm{O}_{4}, \mathrm{C}$ 69.76, H 5.46. Found C 69.80, H 5.30.

Synthesis of 3-(ethoxycarbonyl)-4-methyl-6-benzyl-2-pyranone (6y): The reaction of $1 \mathbf{y}$ (189 mg, $1.2 \mathrm{mmol}), \mathrm{K}_{2} \mathrm{CO}_{3}(14.0 \mathrm{mg}, 0.1 \mathrm{mmol})$ and diethyl malonate $(160 \mathrm{mg}, 1 \mathrm{mmol})$ in 2 $\mathrm{ml}$ of $\mathrm{CH}_{2} \mathrm{Cl}_{2}$ afforded $225 \mathrm{mg}(69 \%)$ of $\mathbf{6 y}$ : white solid, recrystalized from $\mathrm{CH}_{2} \mathrm{Cl}_{2} /$ hexane(1:20), m.p. 59.5-60.5 ${ }^{\circ} \mathrm{C}$; IR 1749, 1683, 1635, $1240 \mathrm{~cm}^{-1} ;{ }^{1} \mathrm{H}$ NMR $(300 \mathrm{MHz}$, $\left.\mathrm{CDCl}_{3}\right) \delta 7.30-7.12(\mathrm{~m}, 5 \mathrm{H}), 5.70(\mathrm{~s}, 1 \mathrm{H}), 4.23(\mathrm{q}, J=7.1 \mathrm{~Hz}, 2 \mathrm{H}), 3.69(\mathrm{~s}, 2 \mathrm{H}), 2.10(\mathrm{~s}, 3$ H), $1.27(\mathrm{t}, J=7.1 \mathrm{~Hz}, 3 \mathrm{H})$; MS $m / z(\%) 273\left(\mathrm{M}^{+}+1,9\right), 272\left(\mathrm{M}^{+}, 28\right), 153$ (100); Elemental Analysis calcd for $\mathrm{C}_{16} \mathrm{H}_{16} \mathrm{O}_{4} \mathrm{C} 70.58, \mathrm{H}$ 5.92. Found C 70.63, H 5.83. 


$$
=\gamma_{\mathbf{1 c}}^{n-\mathrm{C}_{6} \mathrm{H}_{13}}=0
$$

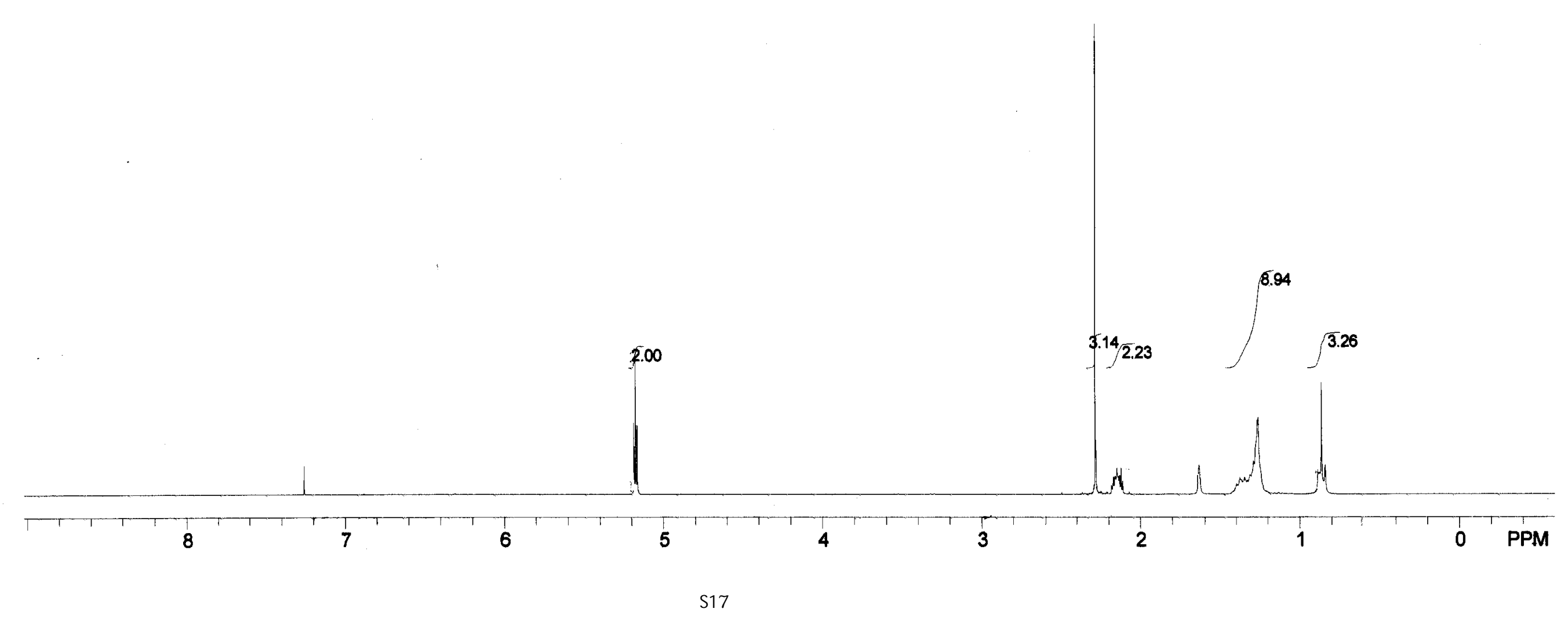




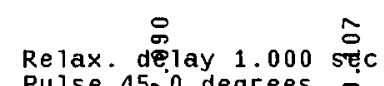
Pulse 45no degrees

Acq timg $1.500 \mathrm{sec}$

2508 repetitions

OBSERVE C13, 75.4420774 MH

DECOUPLE H1, $300.0294698 \mathrm{MHZ}$

Power 40 dB

cont inuous ly on

DATA PROCESSING

Line broadening $1.0 \mathrm{~Hz}$

FT size 65536
Total time $95 \mathrm{hr}, 5 \mathrm{~min}, 35 \mathrm{sec}$

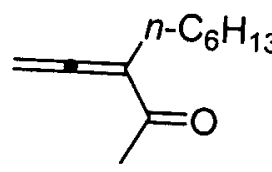

1c

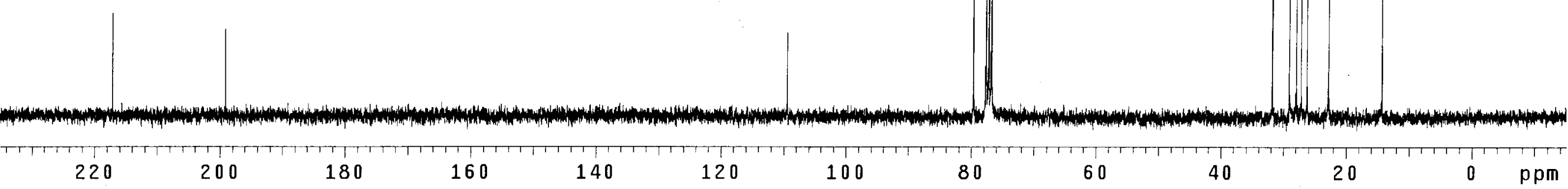



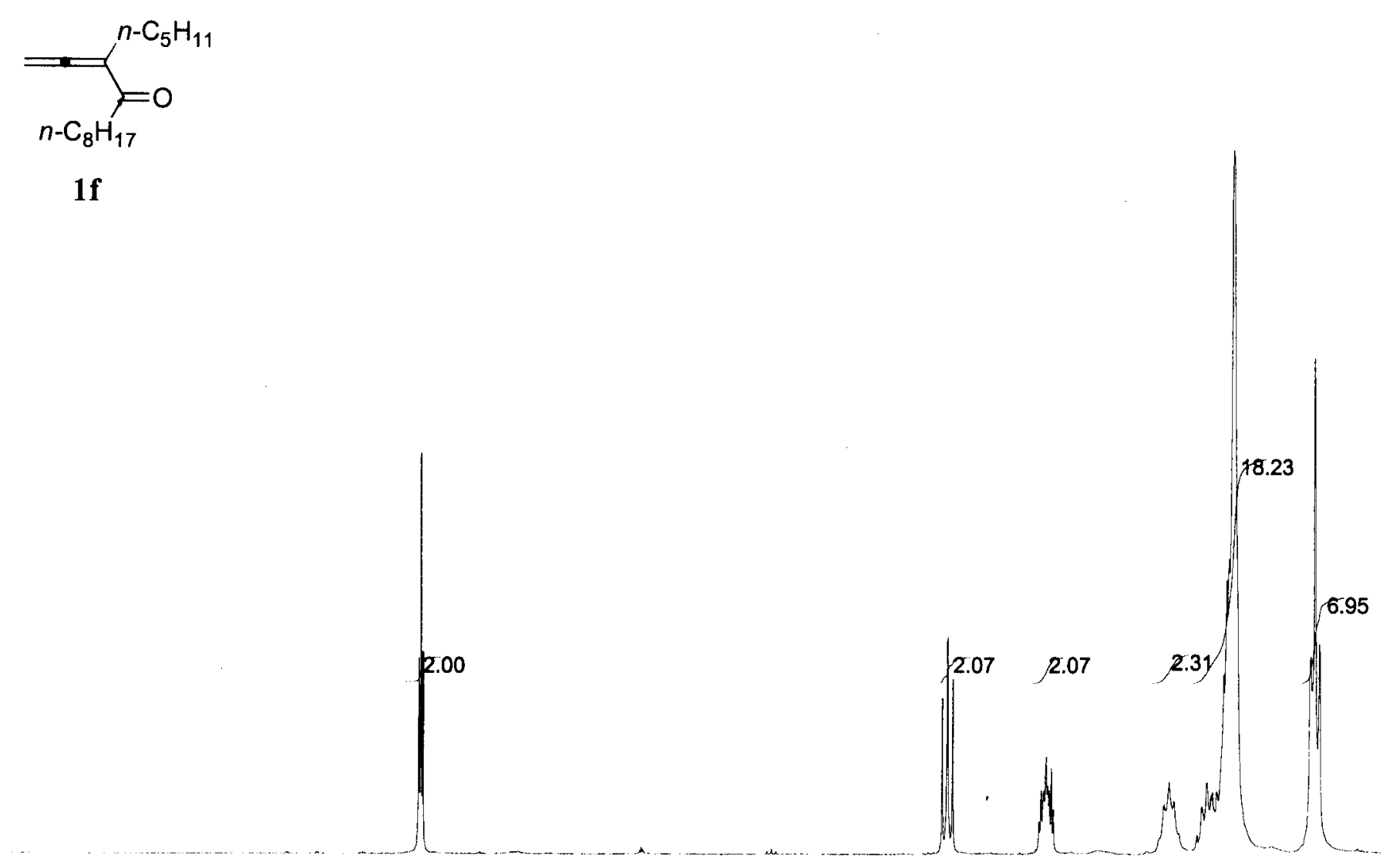


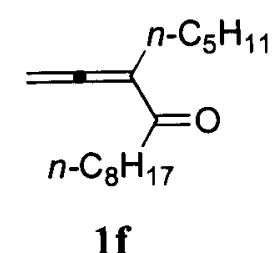

RFF

(1)1) 1)

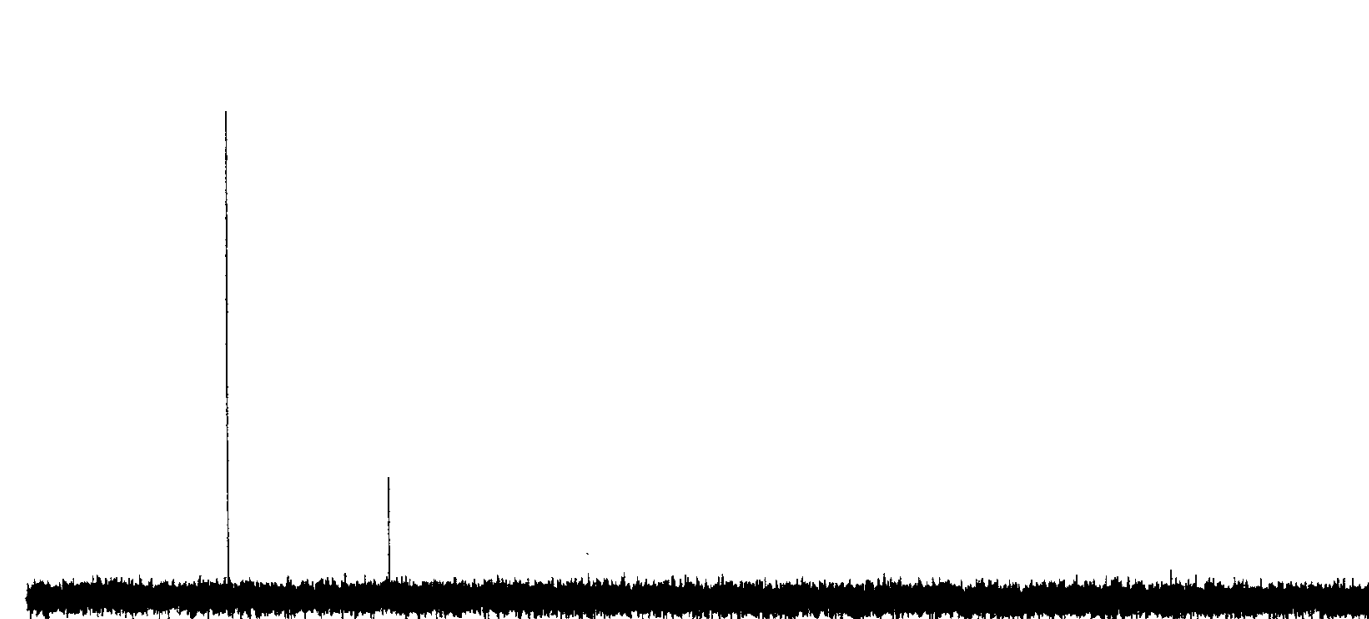




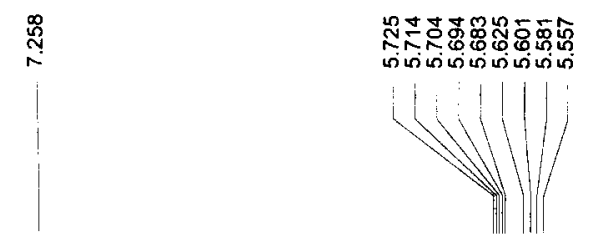

蒿界哥

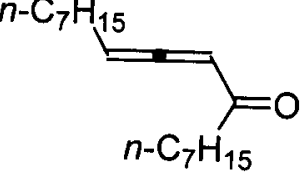

$1 \mathbf{k}$
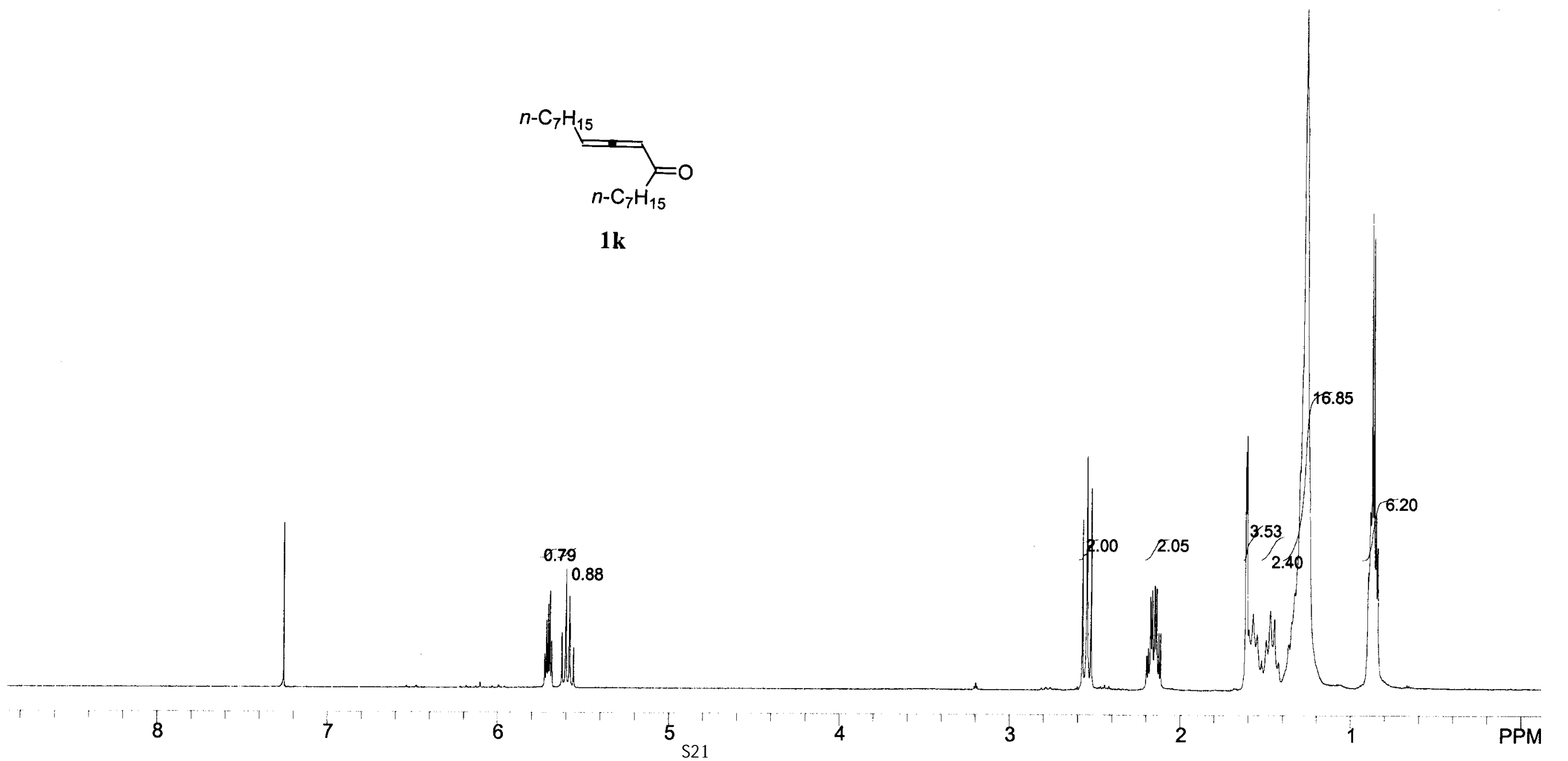
Pulse Sequence: s2pul

Solvent: $\operatorname{CoC} 13$

Ambient temperature
Mercury-300BB "MERCURYv×300"

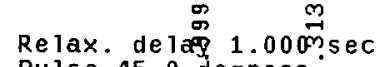

Pulse 45.0 degreesn

Acq time 1500 se

290 repetitions

$.4420774 \mathrm{MHz}$

DECOUPLE H1 $300.0294698 \mathrm{MHZ}$

Power $40 \mathrm{~dB}$.

cont inuously on

WALT2-16 modulated

DATA PROCESING

FT size 65536

Total time olmin, $0 \mathrm{sec}$

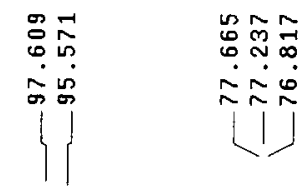

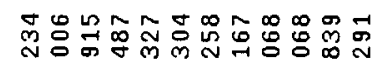

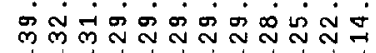

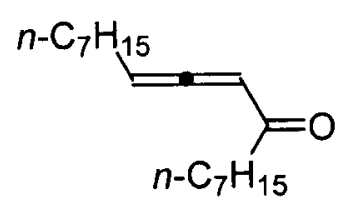

$1 k$

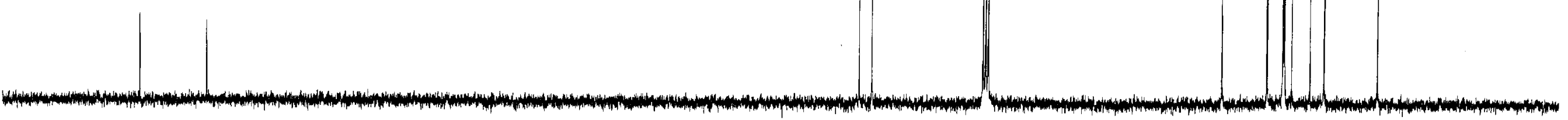



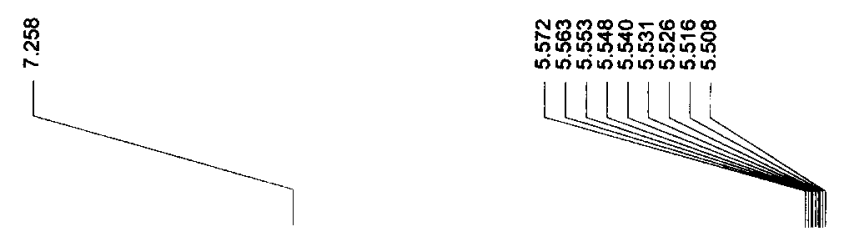

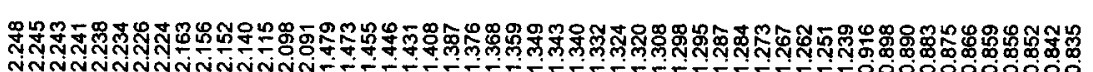
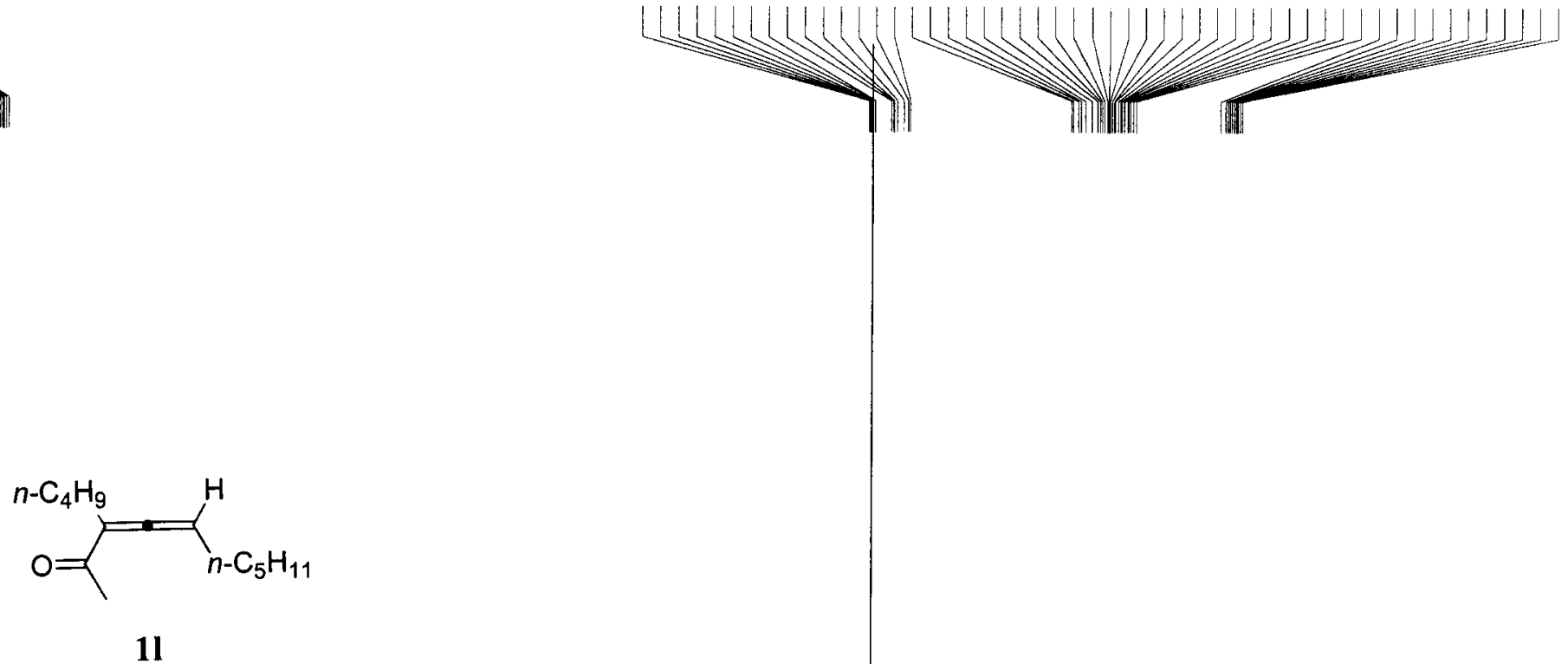

7

ytili

0.90

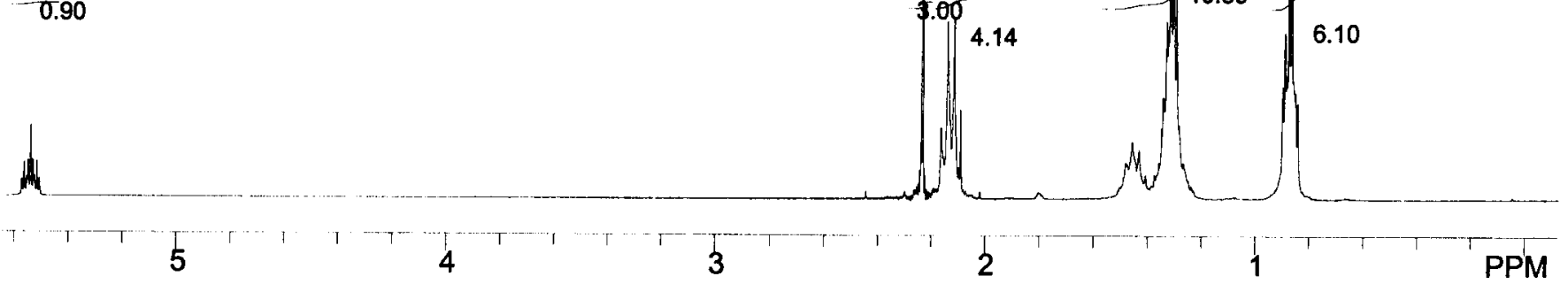



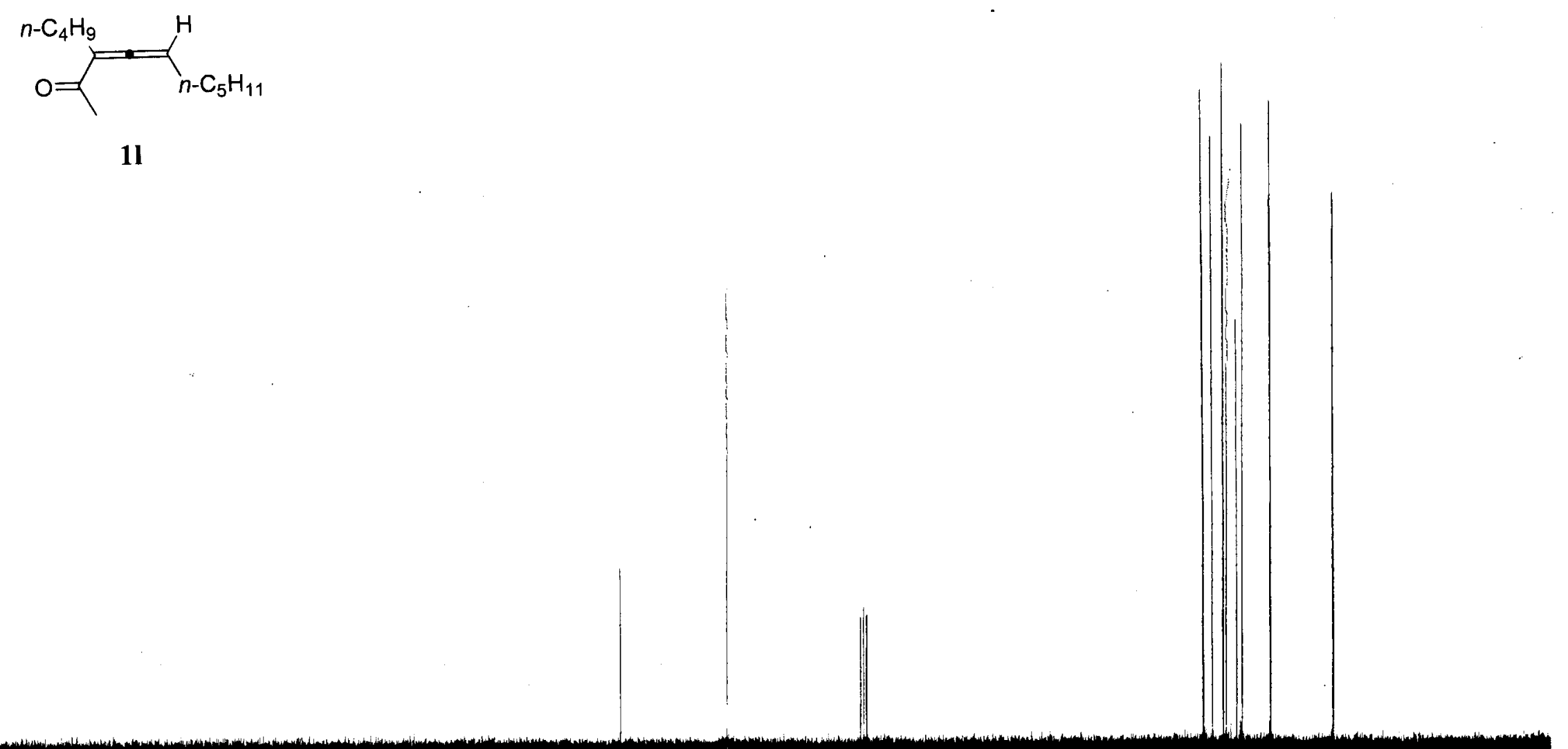


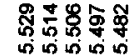

WU

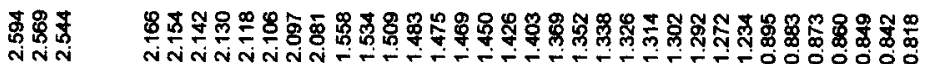

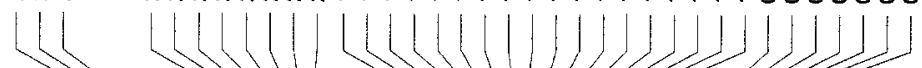

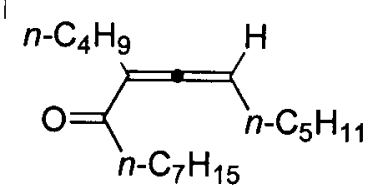

$1 \mathrm{~m}$

0.83
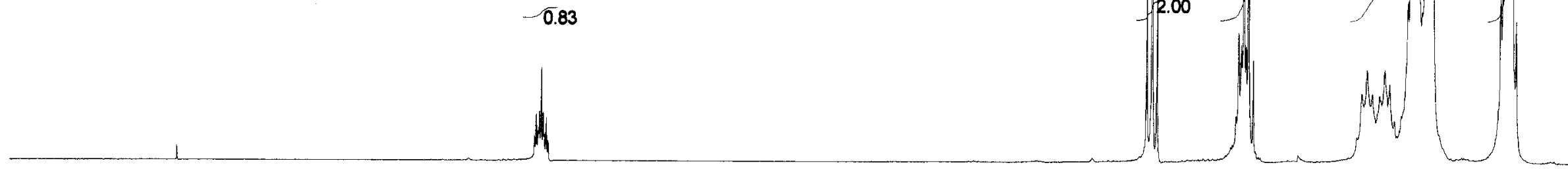


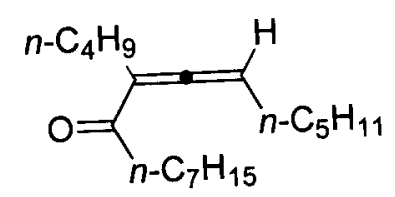

$1 \mathrm{~m}$

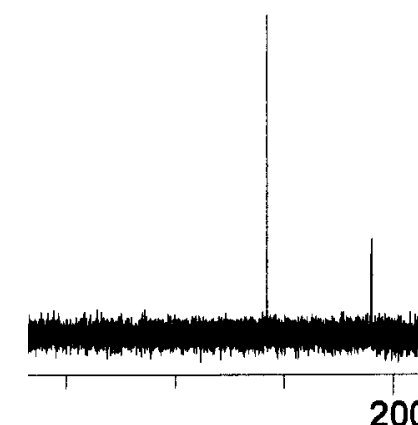



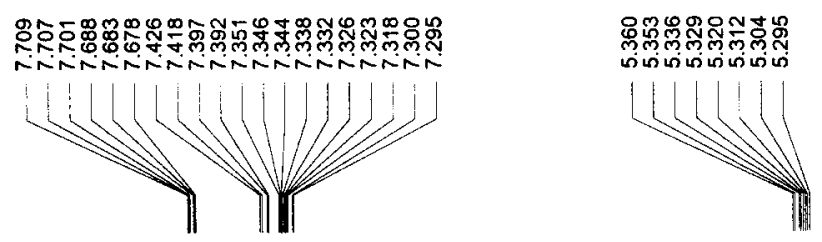

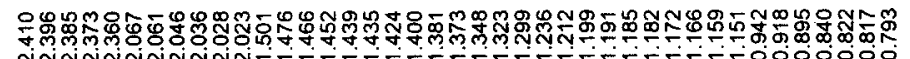
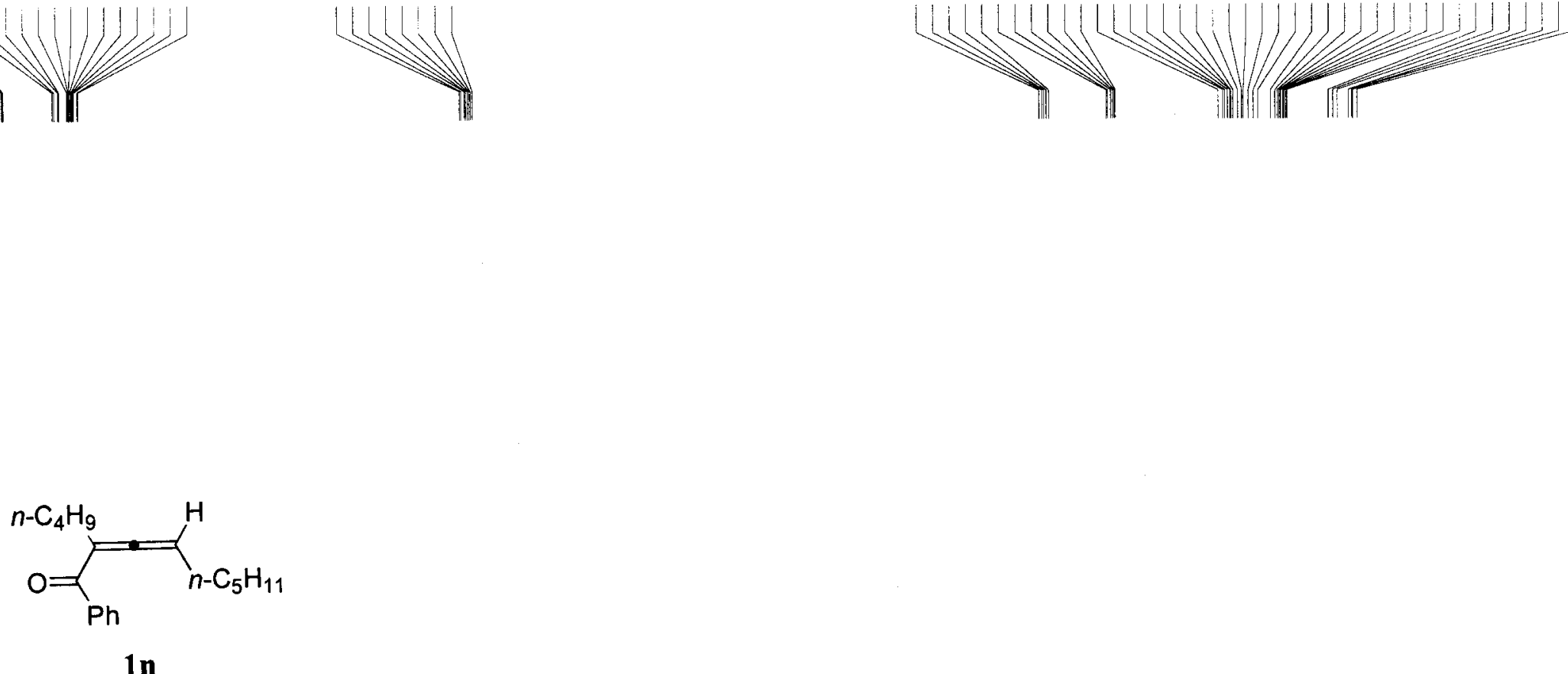

$1 n$

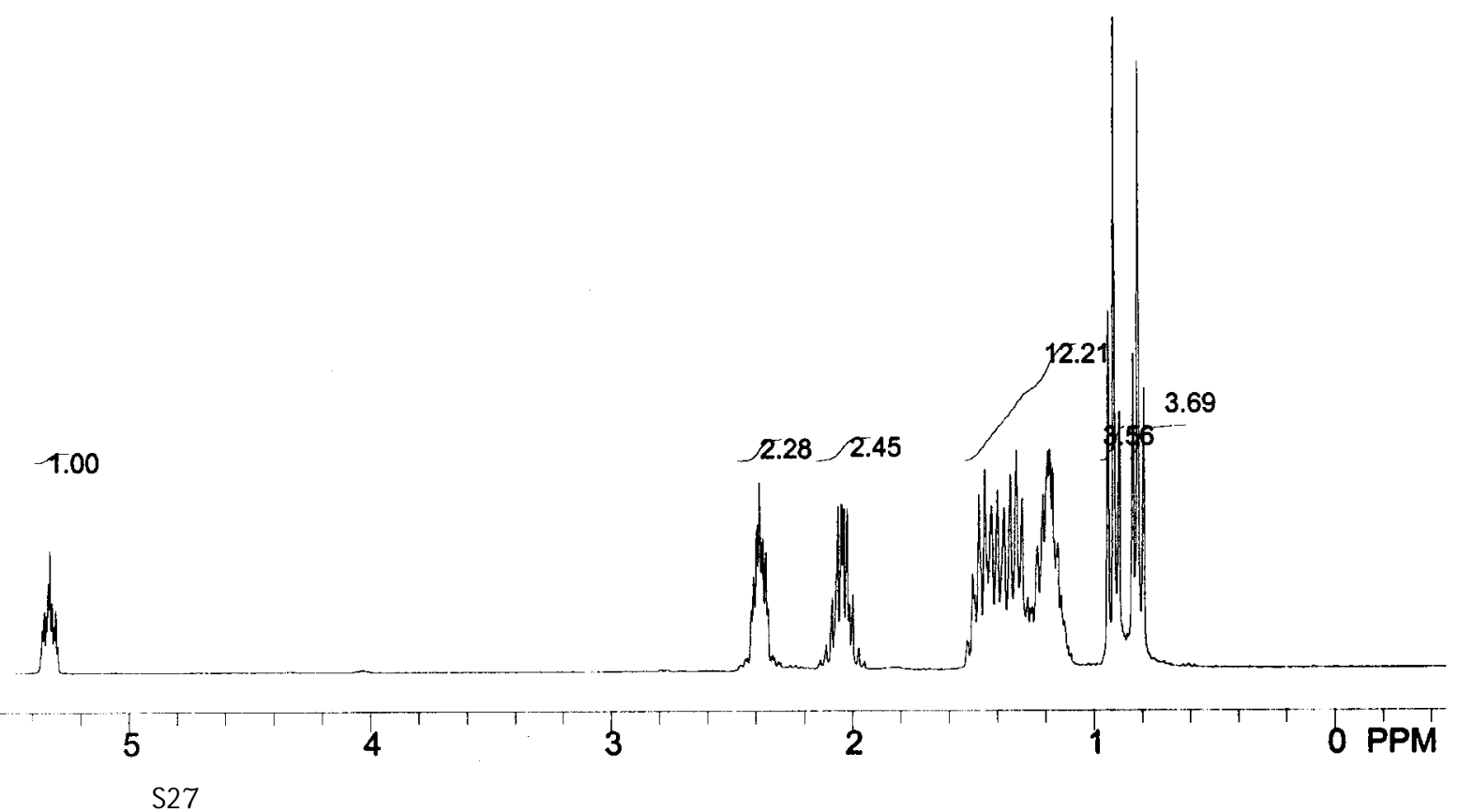




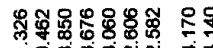

n- $\mathrm{C}_{4} \mathrm{H}_{9}$

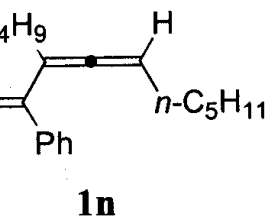

1n 

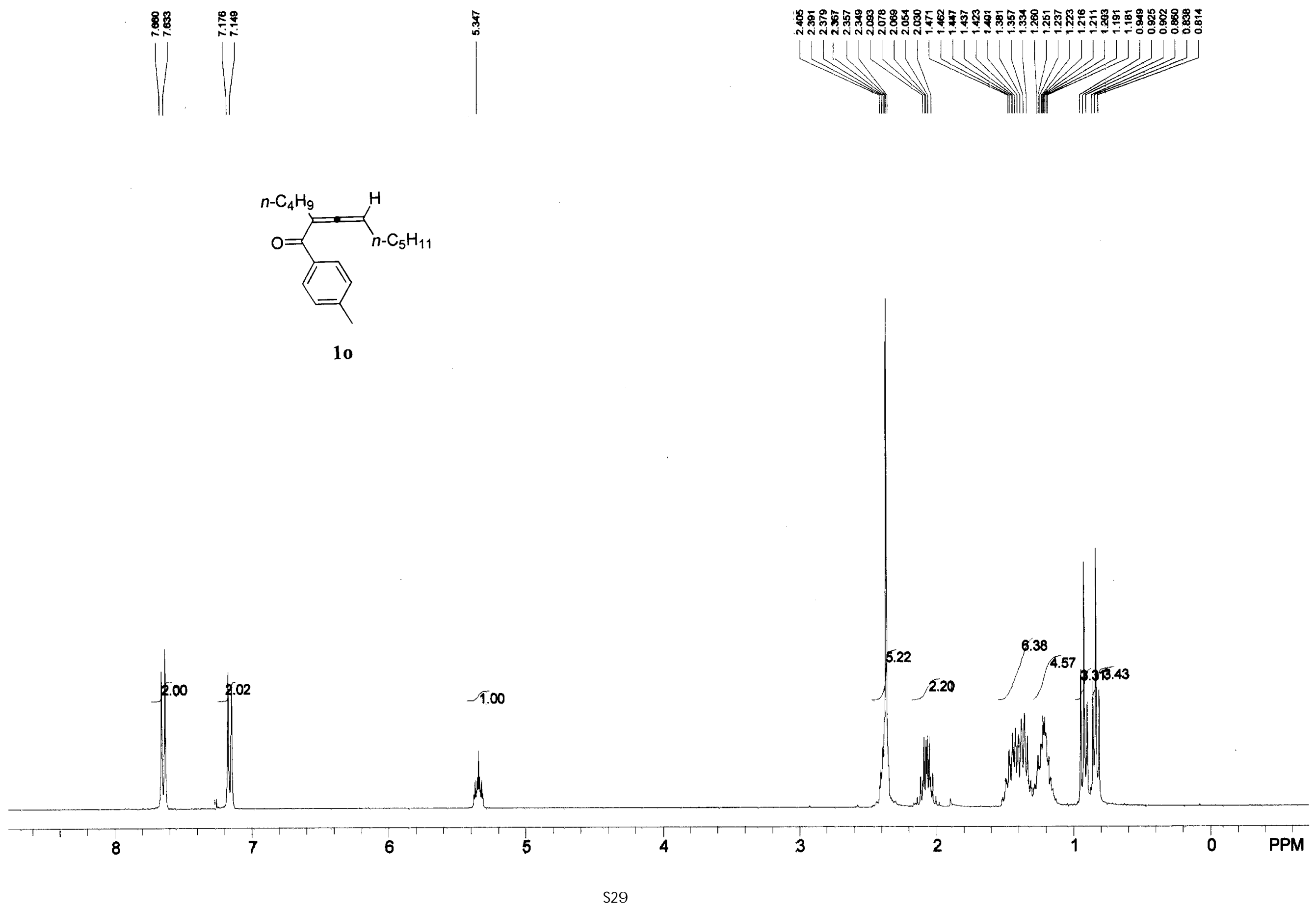

10

HH

$\leqslant+1$

iin $\mid$ || 


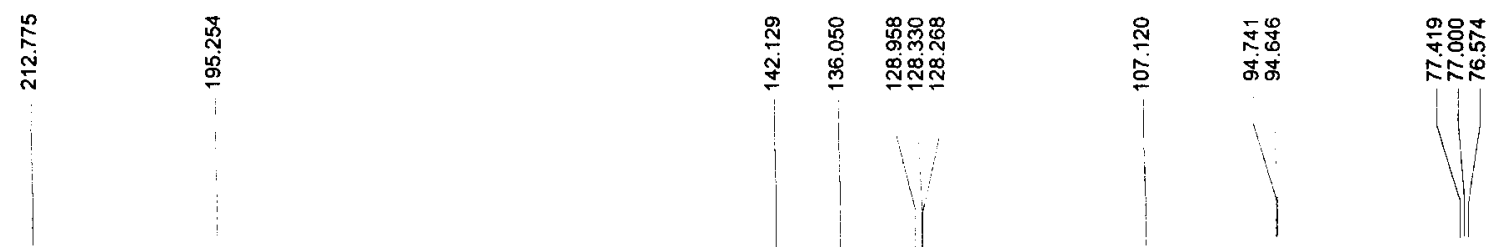

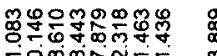

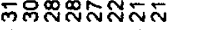

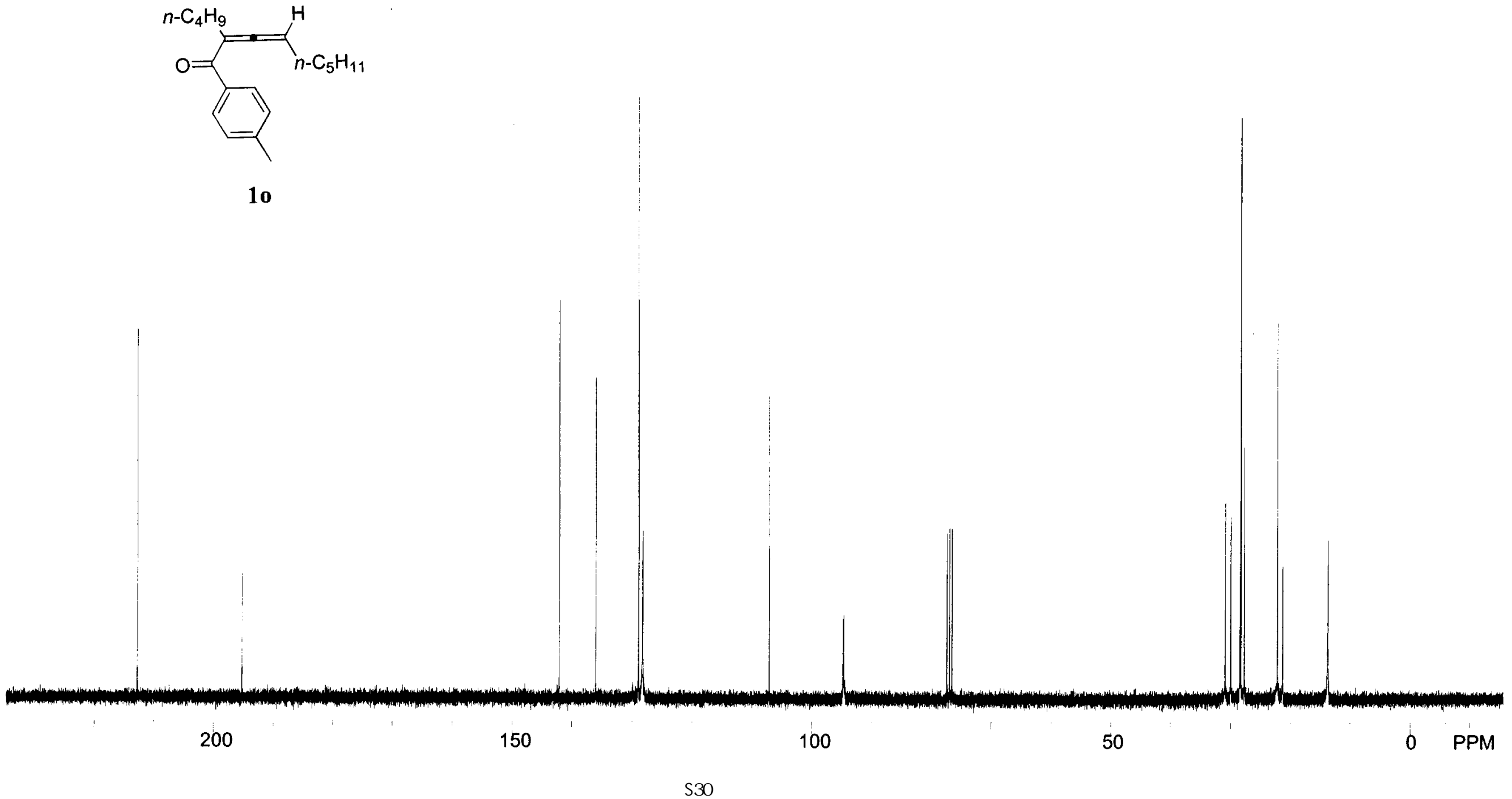

10

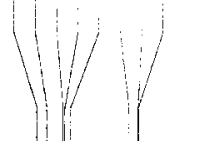




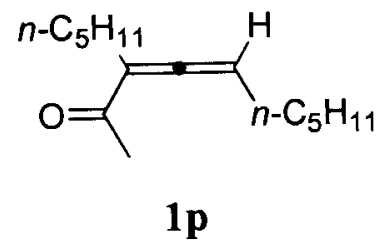

0.91 

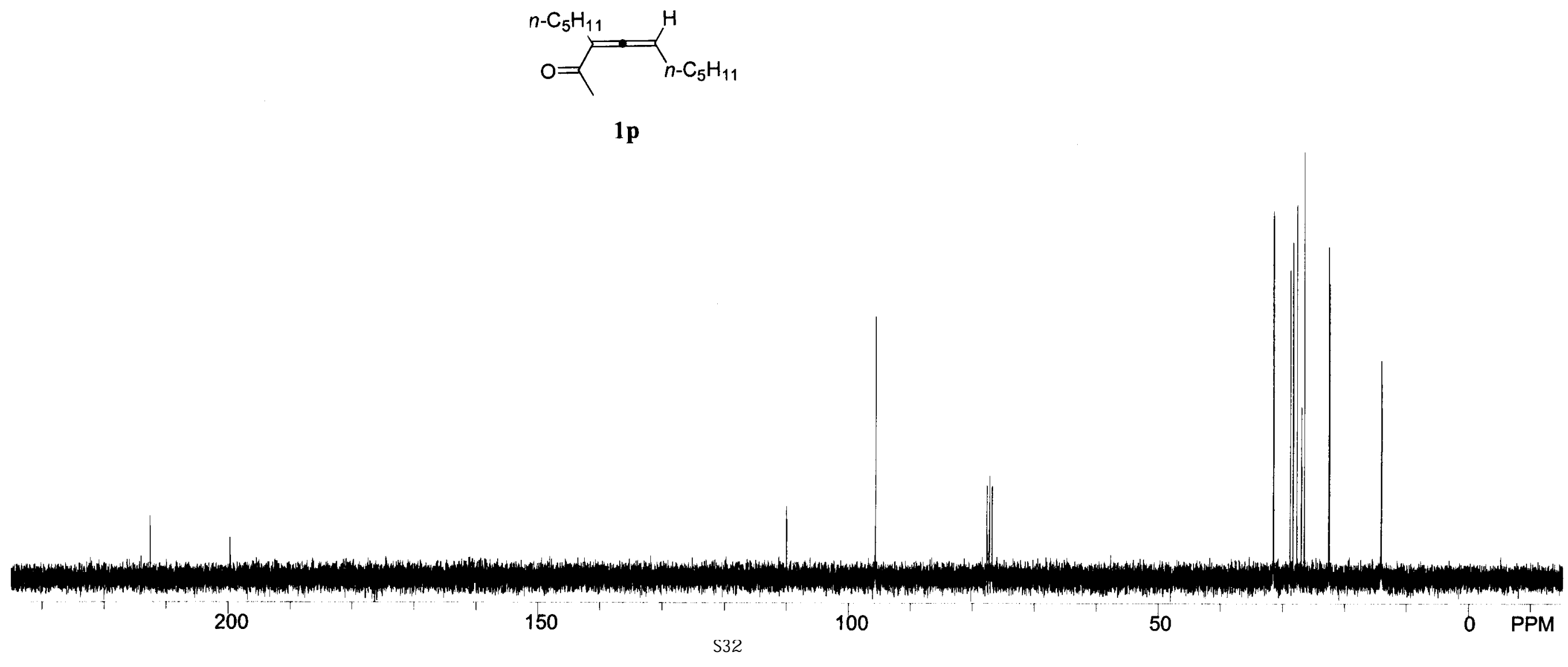


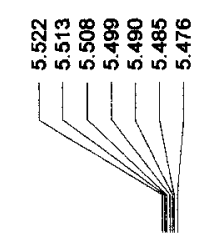

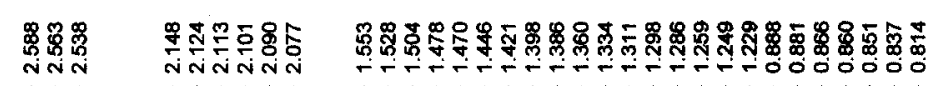

$\mathrm{O}=\underbrace{n-\mathrm{C}_{5} \mathrm{H}_{11}}_{n-\mathrm{C}_{7} \mathrm{H}_{15}}$

$1 q$

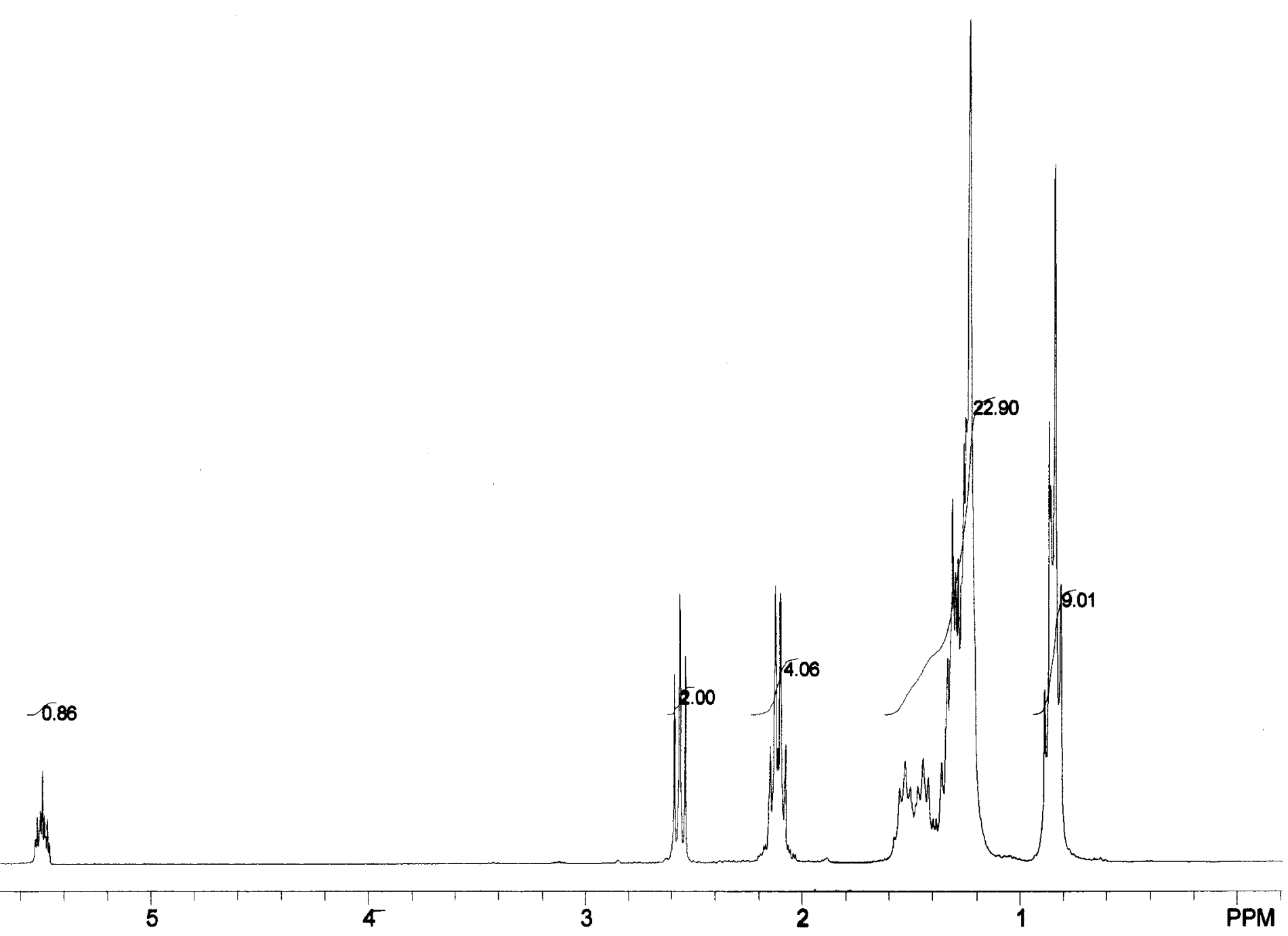




$$
\text { O= }
$$

$1 \mathbf{q}$ 


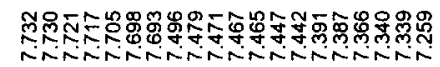

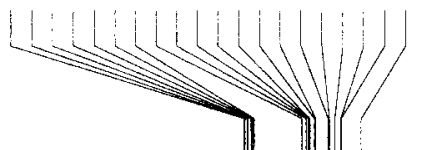

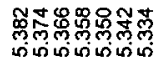

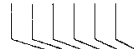

$1 \mathbf{r}$

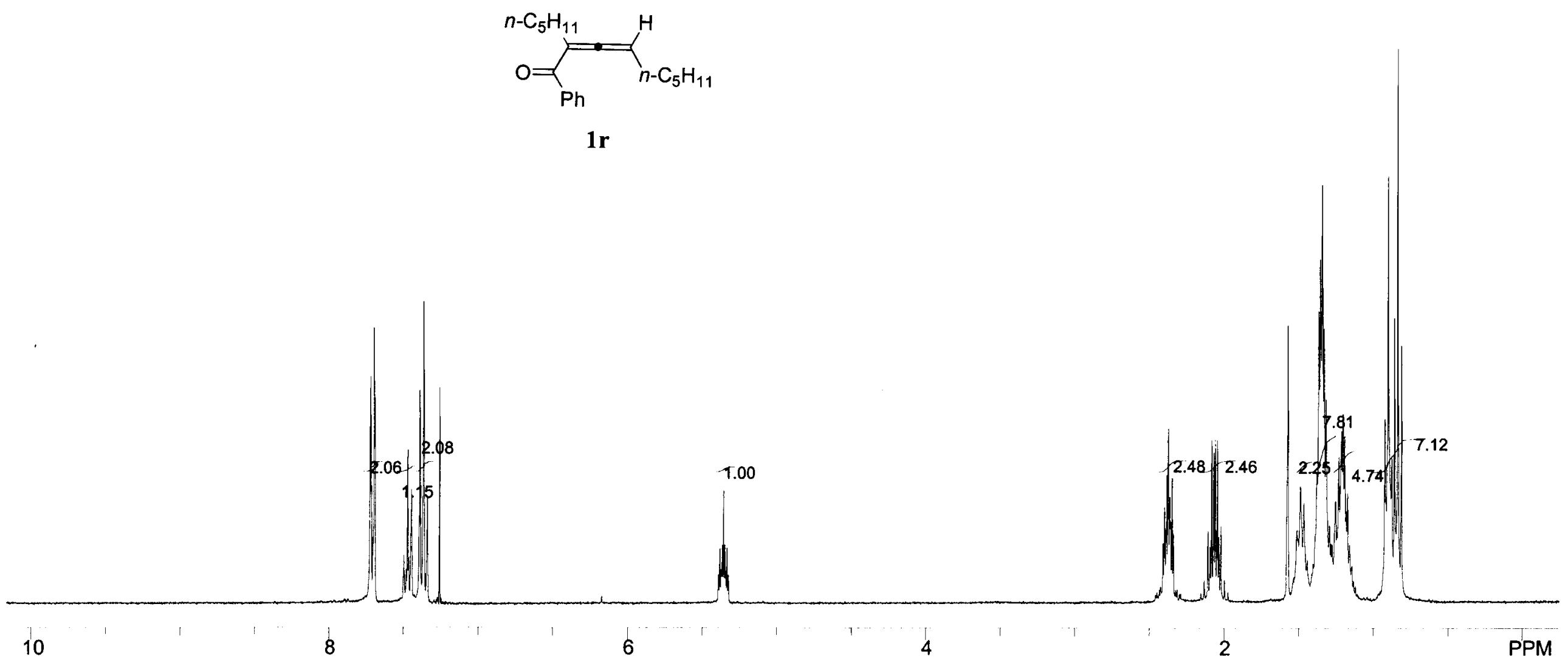

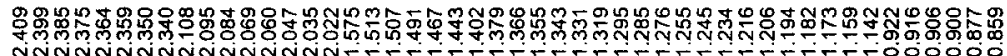

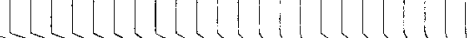

$\ldots=-\ldots(1))$ 

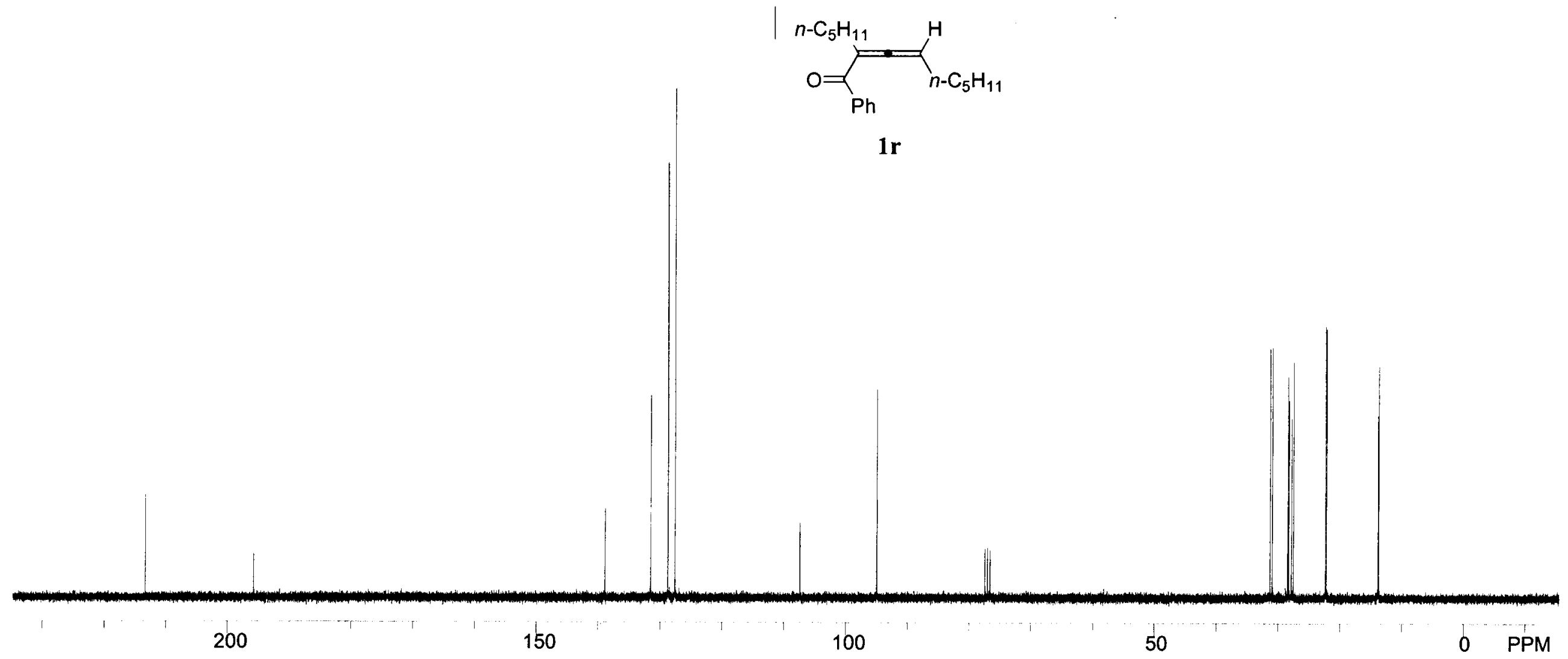

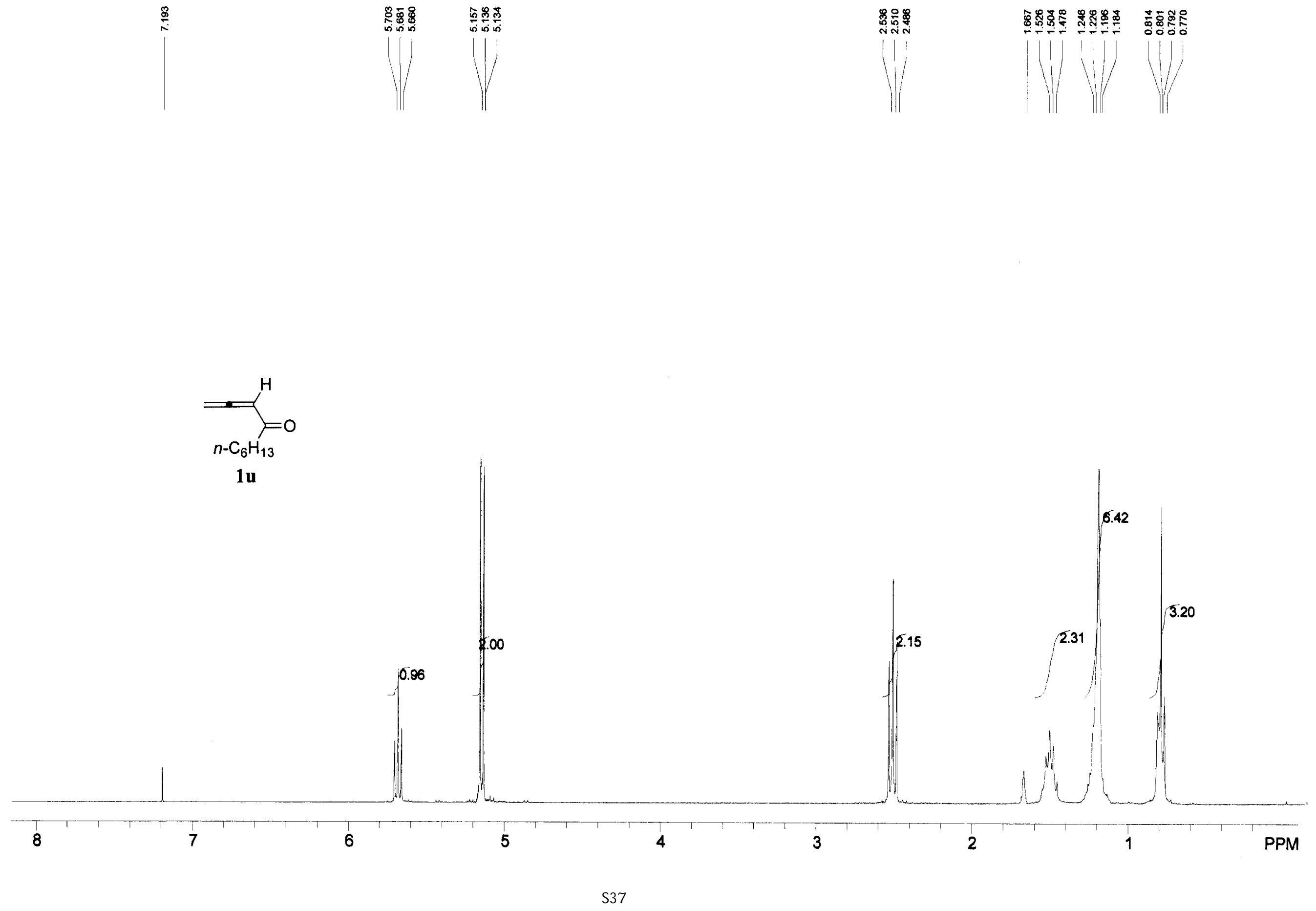


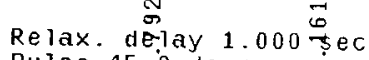
Pulse 4500 degrees

Acq time 2.000 se

48 repetitions

OBSERVE C13,75.4420774 MHZ

DECOUPLE H1, $300.0294698 \mathrm{MHZ}$

Power $42 \mathrm{~dB}$

cont inuously on

DATA PROCESSINO

Line broadening

otal time $116 \mathrm{hr}, 41 \mathrm{~min}, 19 \mathrm{sec}$

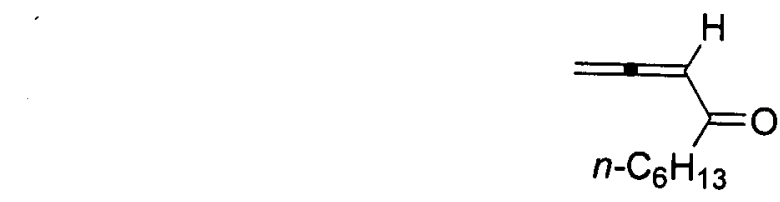

$1 \mathbf{u}$
क

\& กลก゚

1

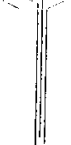

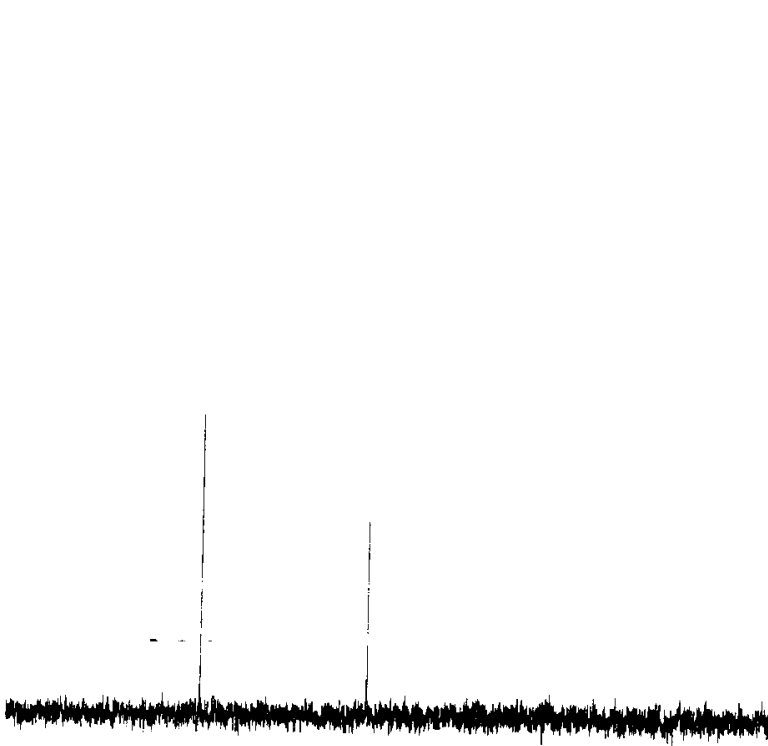

And 


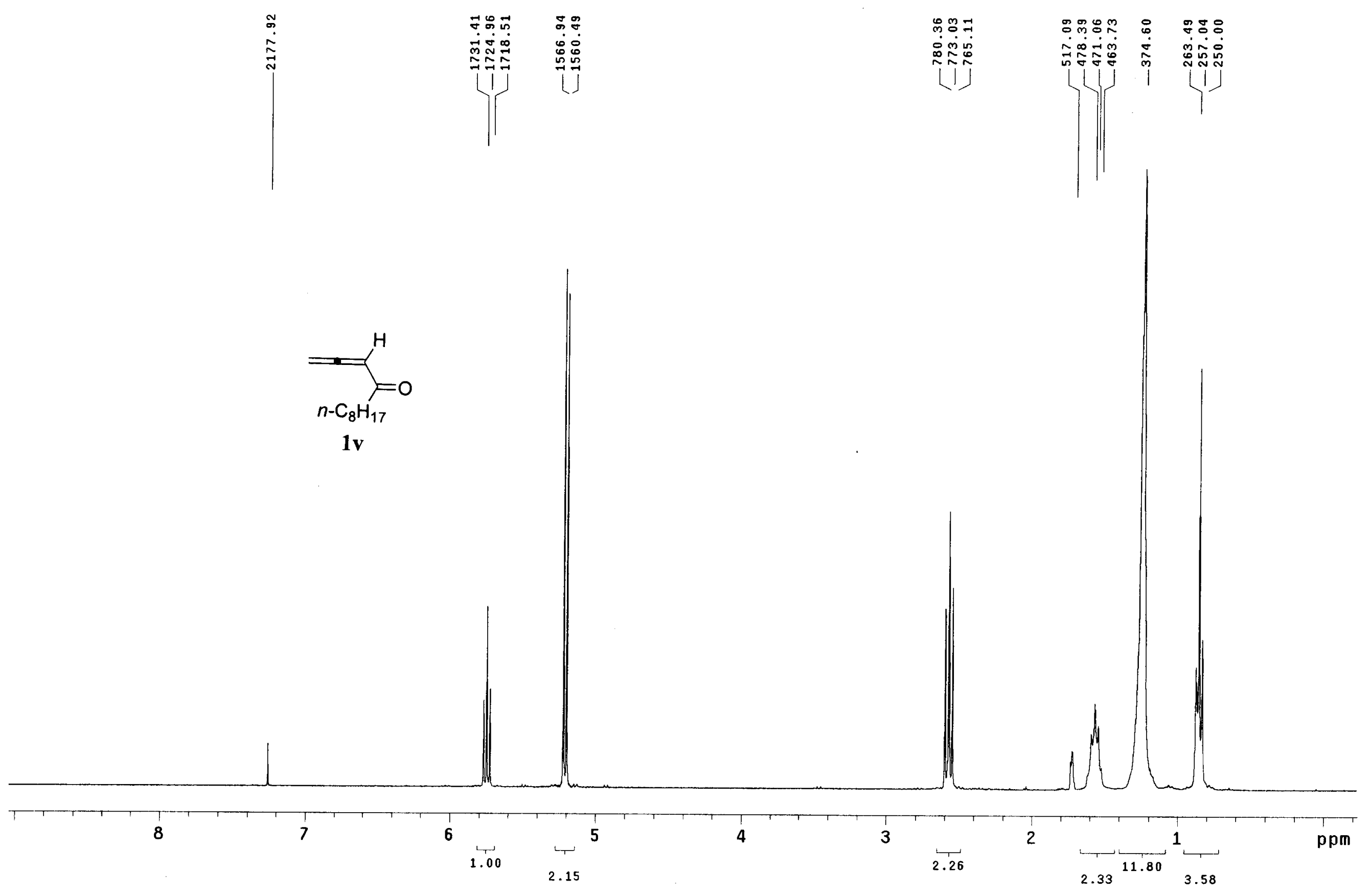




$$
=\sum_{n-\mathrm{C}_{8} \mathrm{H}_{17}}^{\mathrm{H}}=\mathrm{O}
$$

$1 v$

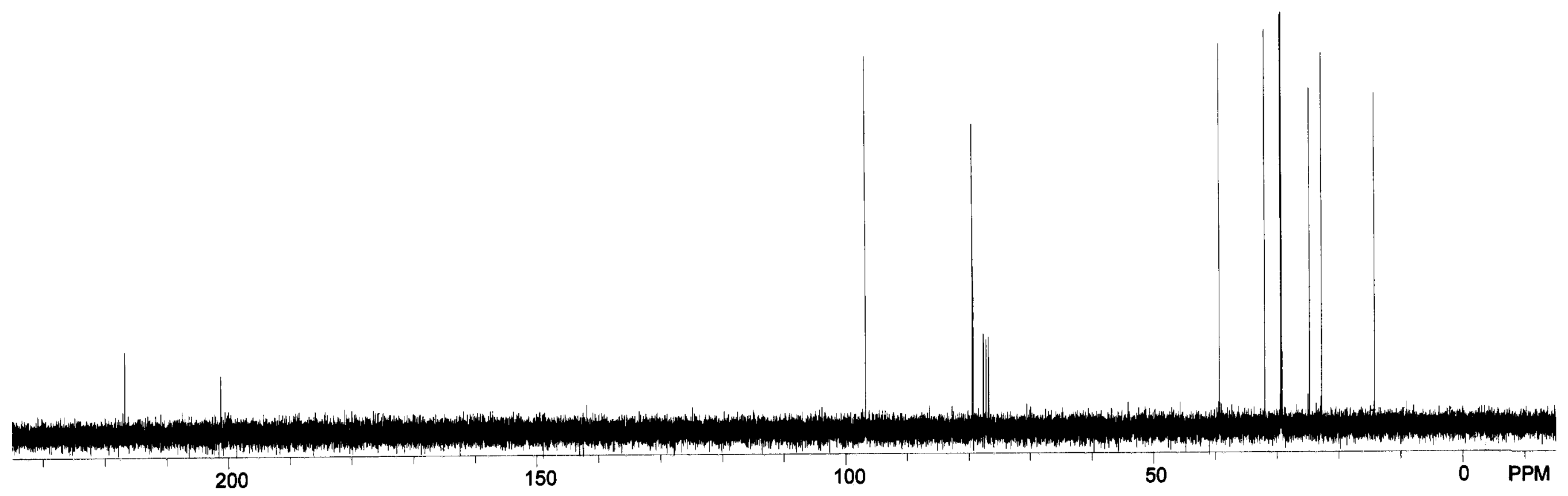


|

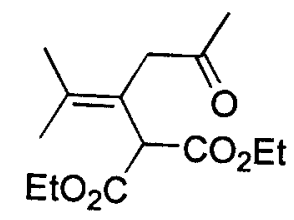

$3 g$

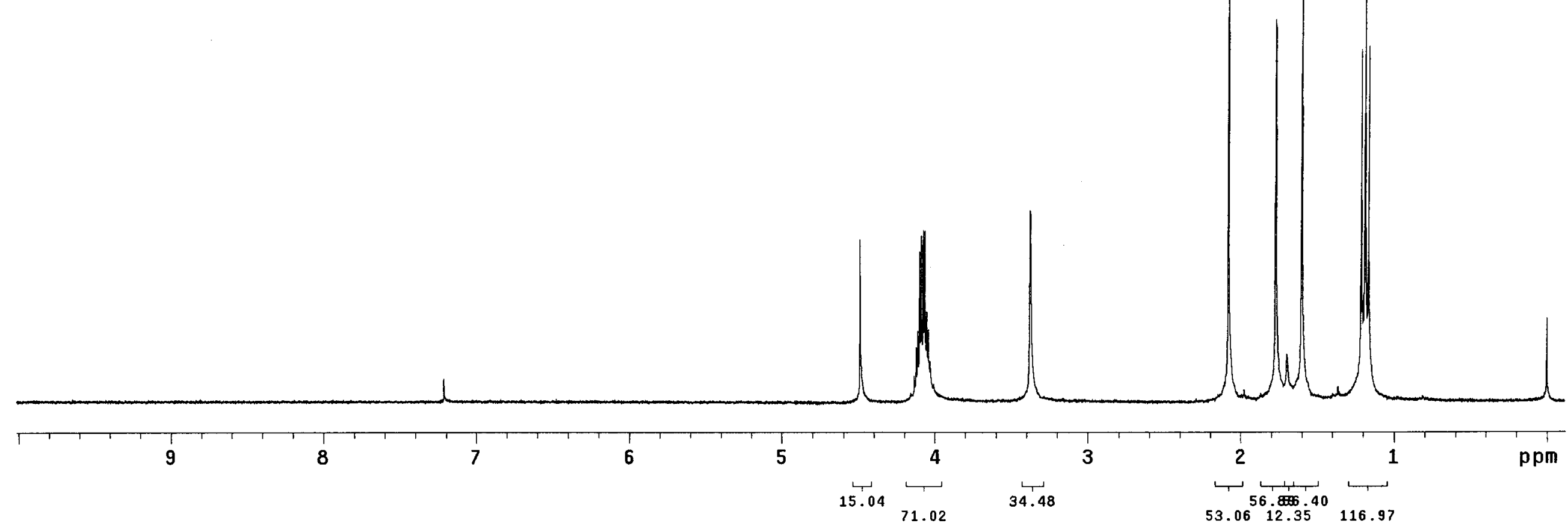


$\mathrm{C} 13 / \mathrm{CDC} r$
ysh-4-130

Pulse Skgence: s2pul

Solvent $\mathrm{EDC} 13$

Ambient temperature

Relax. delay $1.000 \mathrm{sec}$

Acq. $t \mathrm{tme} 3.405 \mathrm{sec}$

Width $18,97.0 \mathrm{~Hz}$

560 repefitions

WBSE

POWP 45 H1, $30.0346884 \mathrm{MHZ}$

on during acquisition

off during delay

WALTZ-16 modulate

FT $51 z e-151072$

Total time $0 \mathrm{~min}, 0 \mathrm{sec}$

\section{范骂}

趈
송

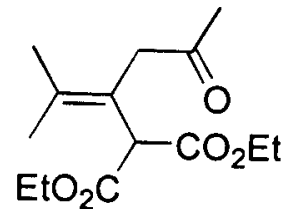

$3 g$

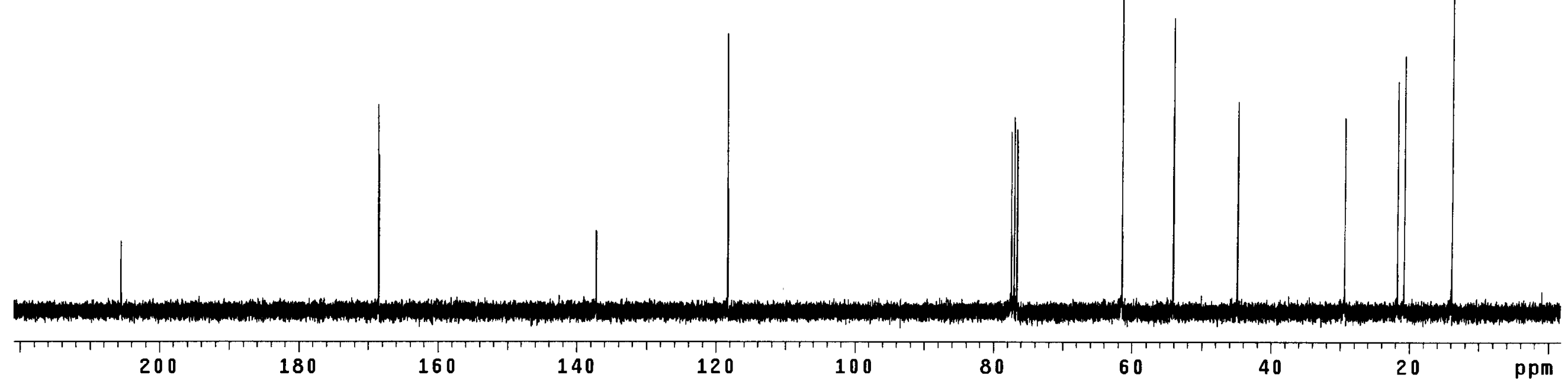




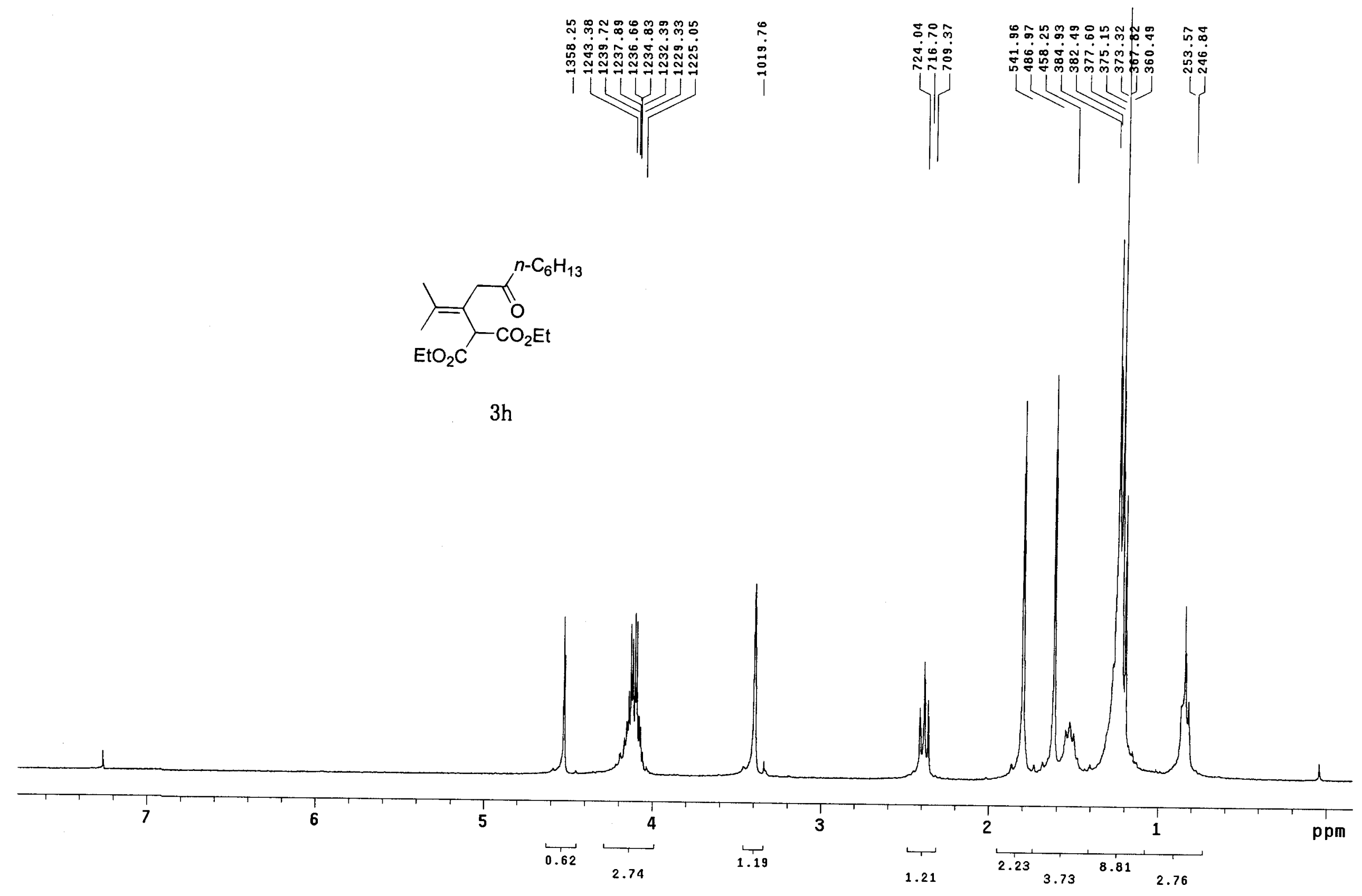


MercurY-300BS "MERCURYVX300"

Relax. de lajy 1.000 sec

Pulse 45.0odegrees

Acq time 2.000 sec

64 repetitipns

64 repetitions

DECOUPLE H1, 300.0294698 MHZ

Power $42 \mathrm{~dB}$

cont inuously on

WAL

Line broadening $1.0 \mathrm{~Hz}$

FT size 131072

Total time $134 \mathrm{hr}, 48 \mathrm{~min}, 51 \mathrm{sec}$

\begin{tabular}{|c|c|c|c|c|}
\hline$\underset{N}{N} \approx$ & $\begin{array}{l}\infty \\
\infty \\
\infty\end{array}$ & $\stackrel{m}{\circ}$ & $\begin{array}{ll}\infty & \infty \\
\infty & \infty \\
N & \sim\end{array}$ & 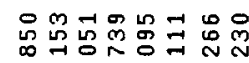 \\
\hline & $\overrightarrow{0}$ & $\sigma$ & & $\vec{m} \underset{N}{N} N \vec{N}$ \\
\hline
\end{tabular}

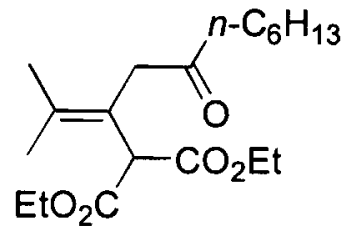

$3 \mathrm{~h}$

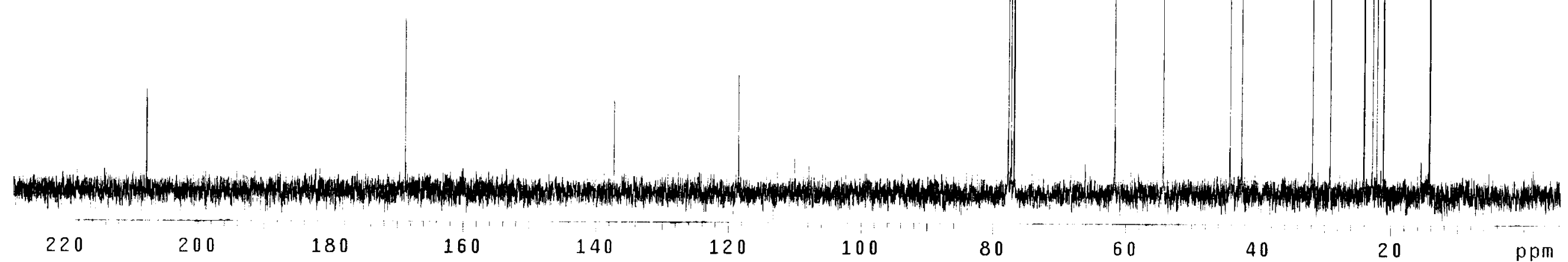



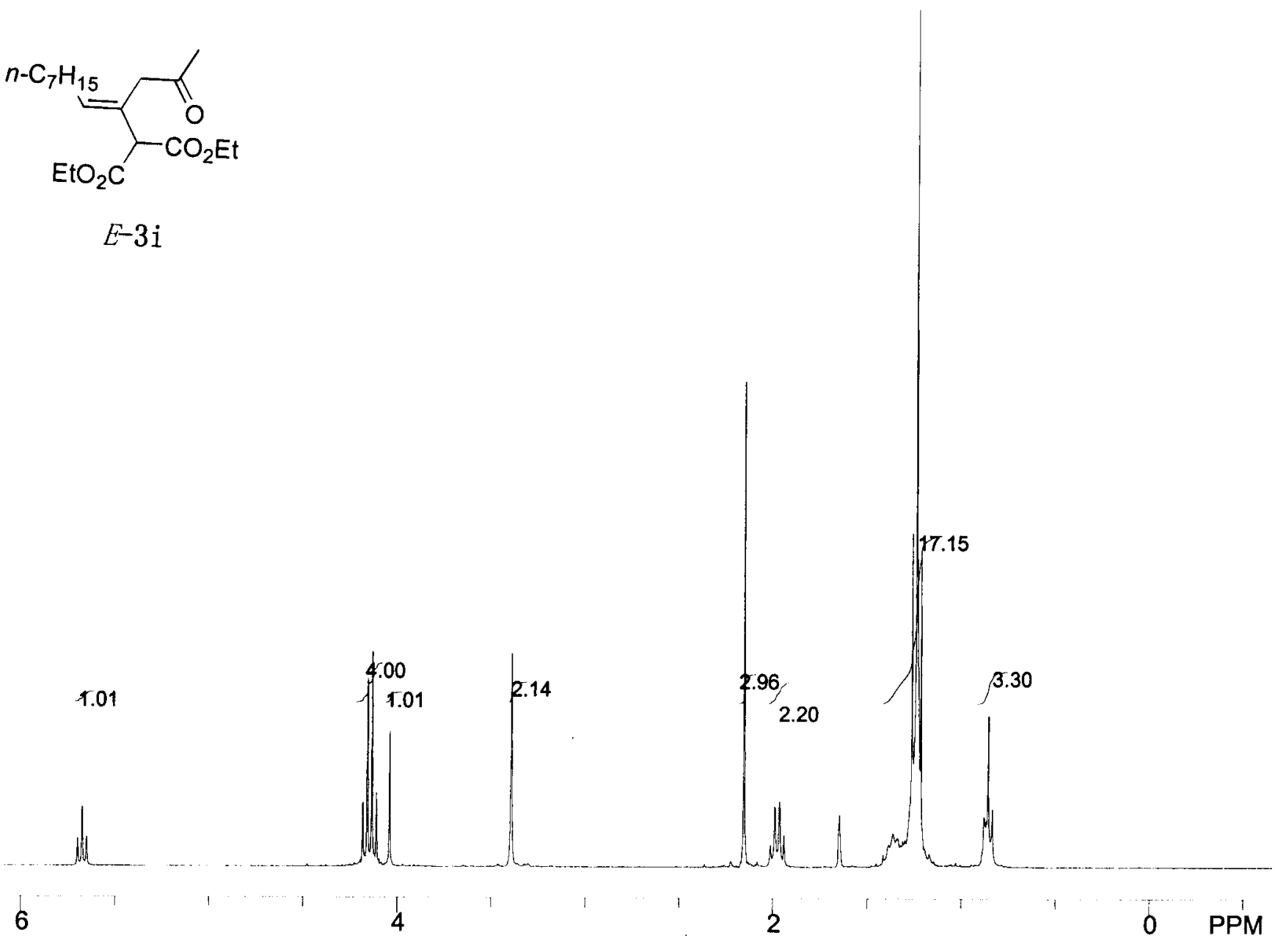
Relax delay $\stackrel{2}{0} 000 \mathrm{sec}$

Pulse 45.0 degrees

Acq. time 2.00
width $30030.0 \mathrm{Tz}$

100 repetitions

OBSERVE C13, $5.4420774 \mathrm{MHz}$

DECOUPLE H1, $300.0294698 \mathrm{MHZ}$

cont inuous ly

WALT Z-16 15 on

DATA PROCESSING

Line broadening $1.0 \mathrm{~Hz}$

Total time $134 \mathrm{hr}, 48 \mathrm{~min}, 51 \mathrm{sec}$

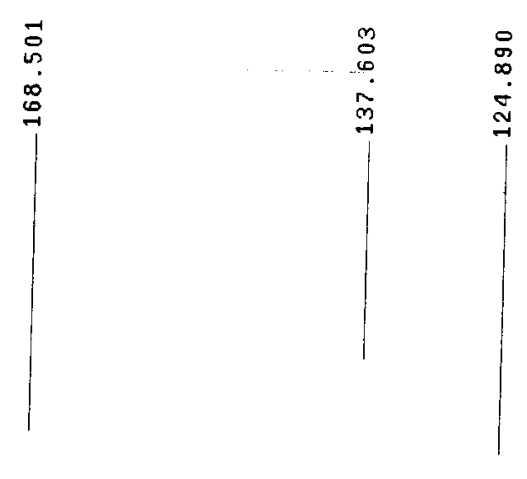
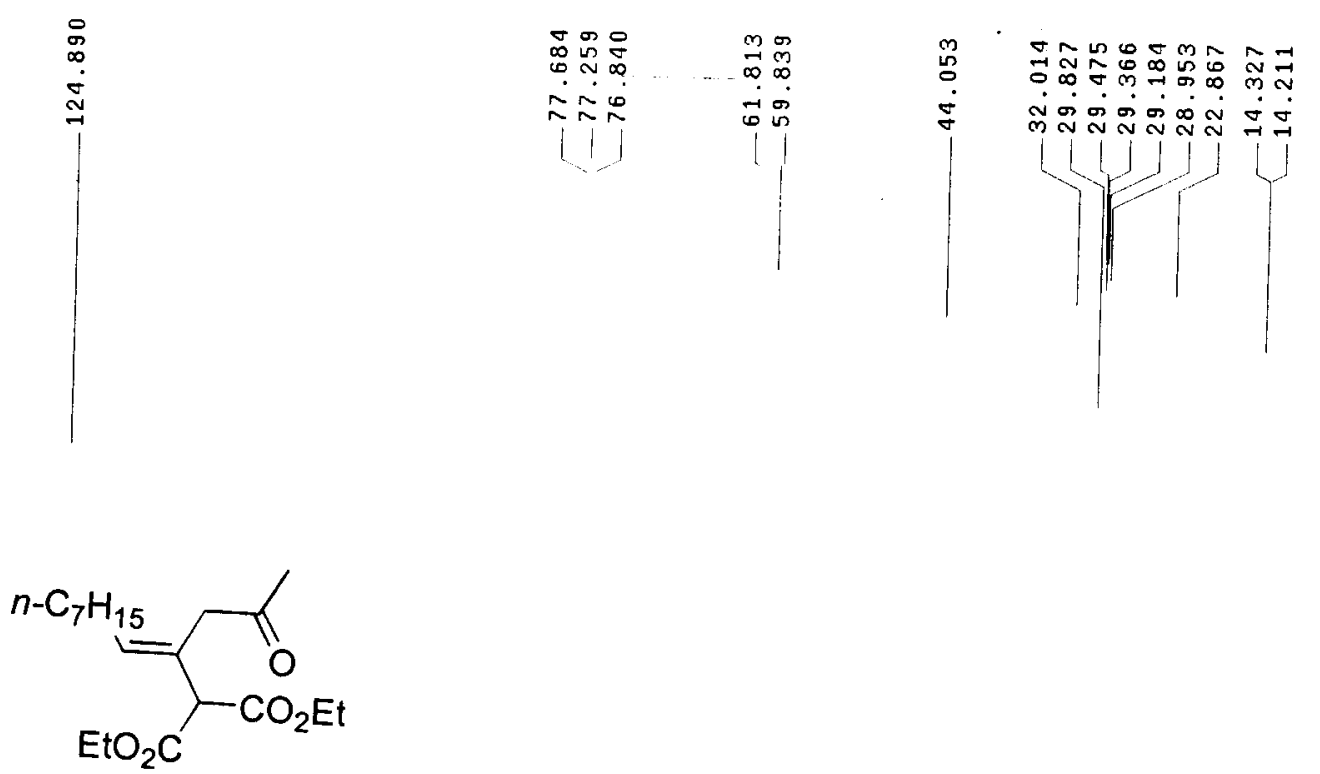

E-3i

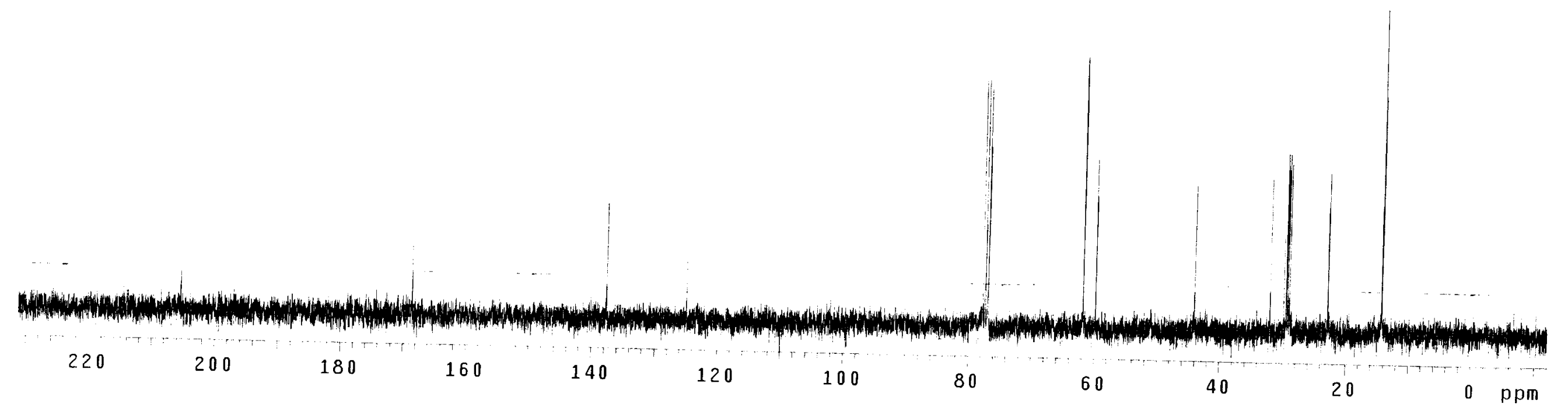




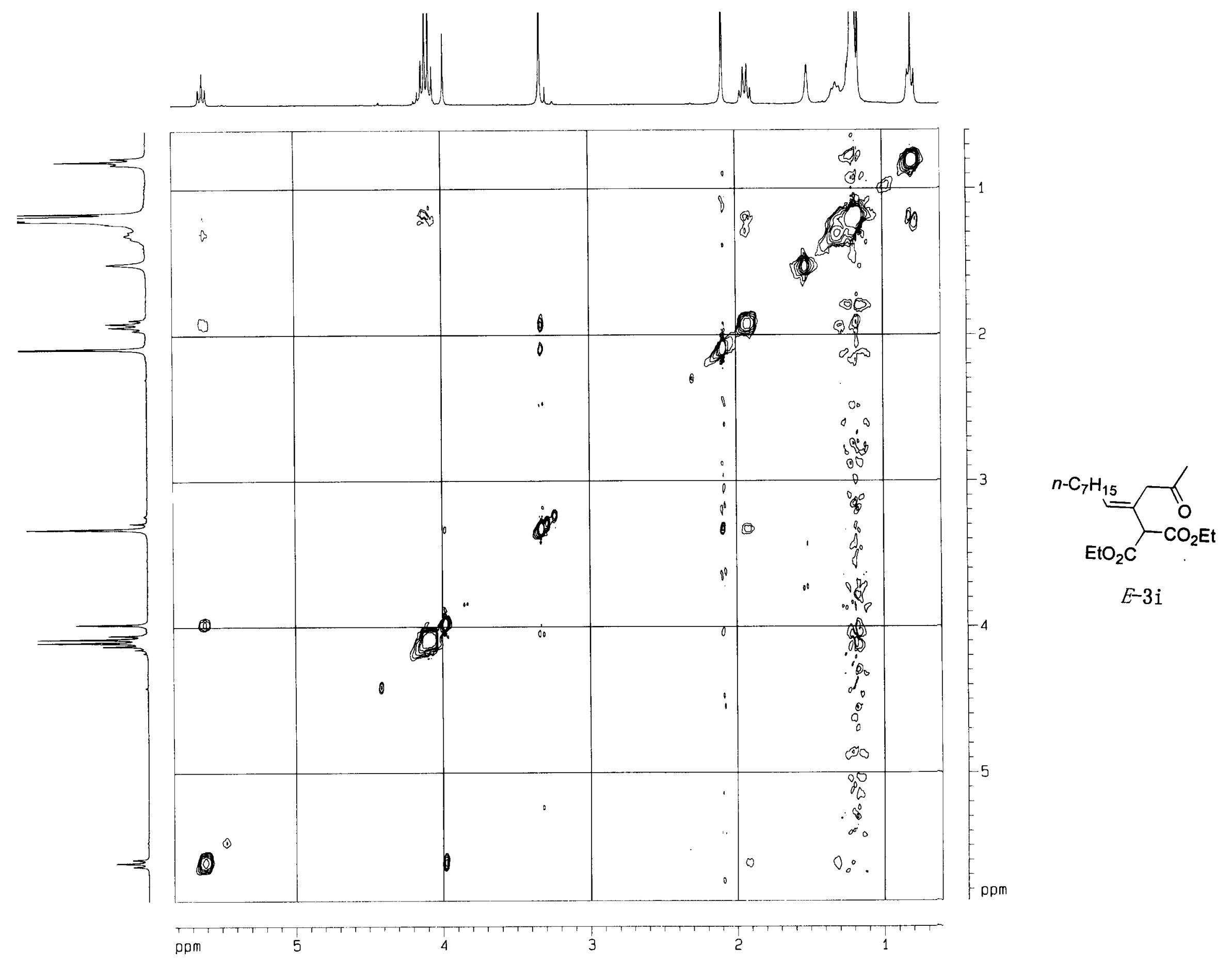




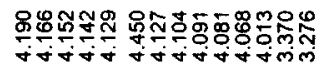

$1+$

$+1$

1

$n-\mathrm{C}_{4} \mathrm{H}$

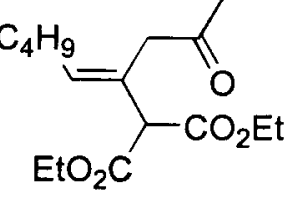

$E^{-3 \mathrm{j}}$

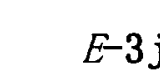
0.02

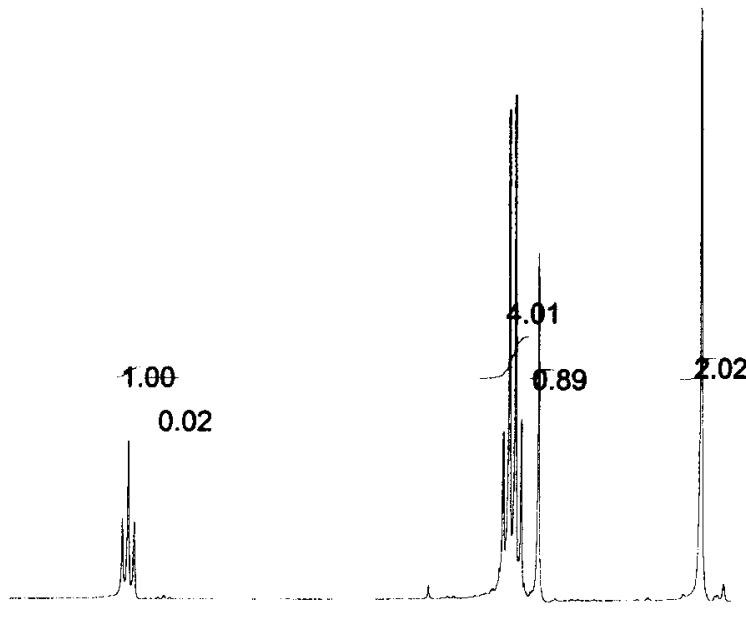

6
4

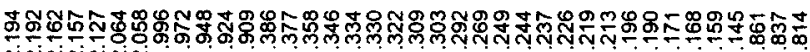

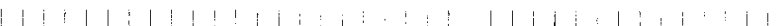

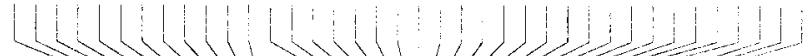
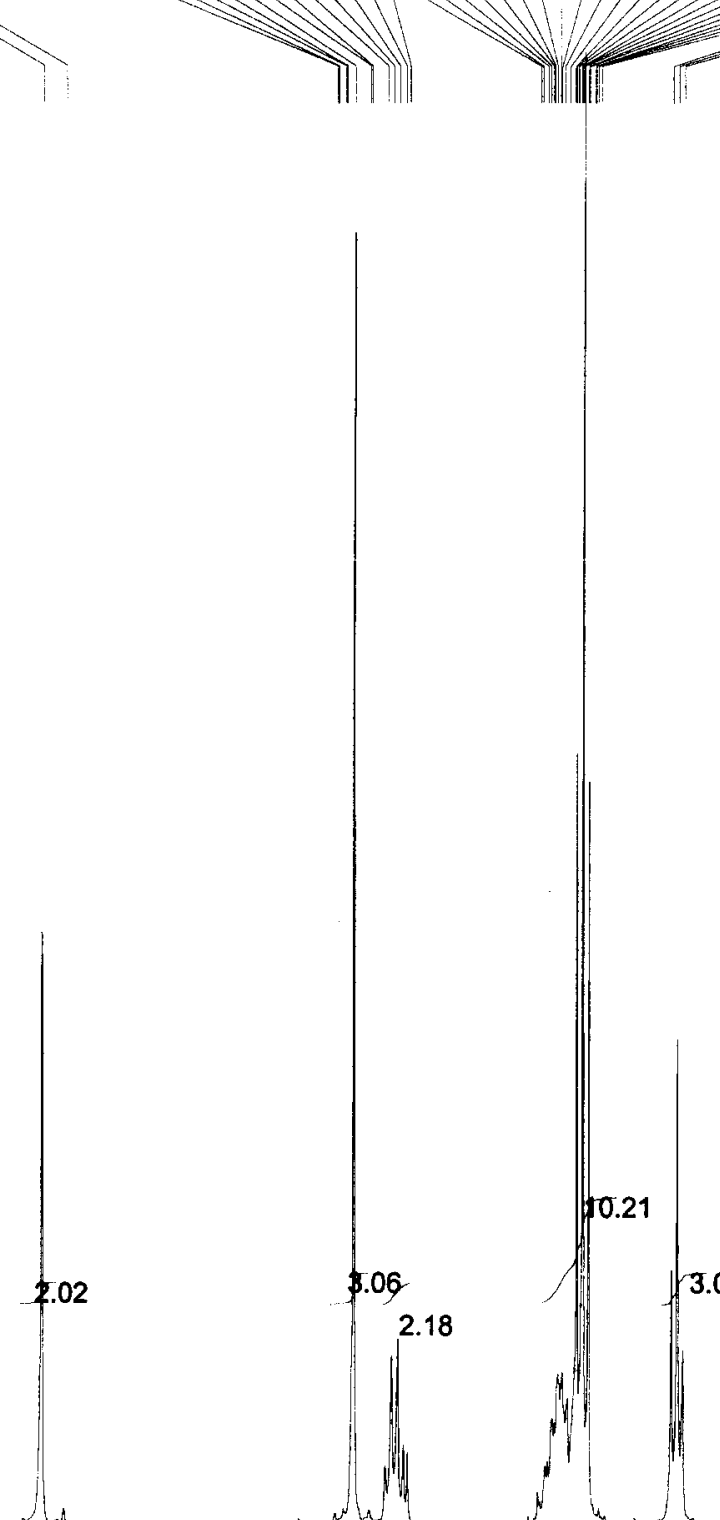

2

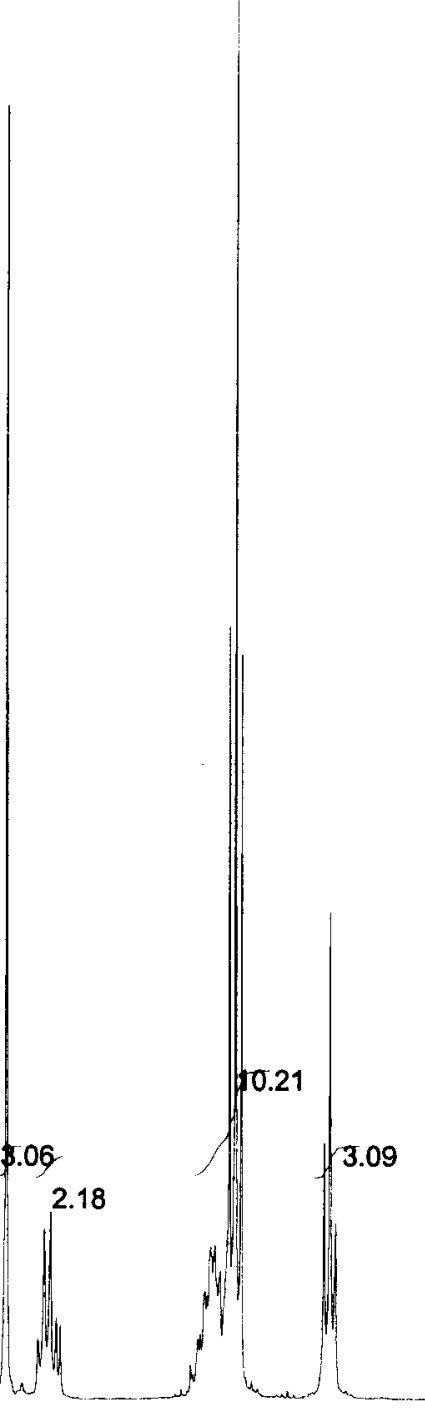




$\mid$

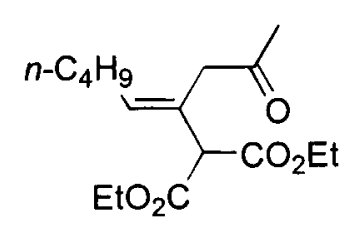

${ }^{-}-3 \mathrm{j}$

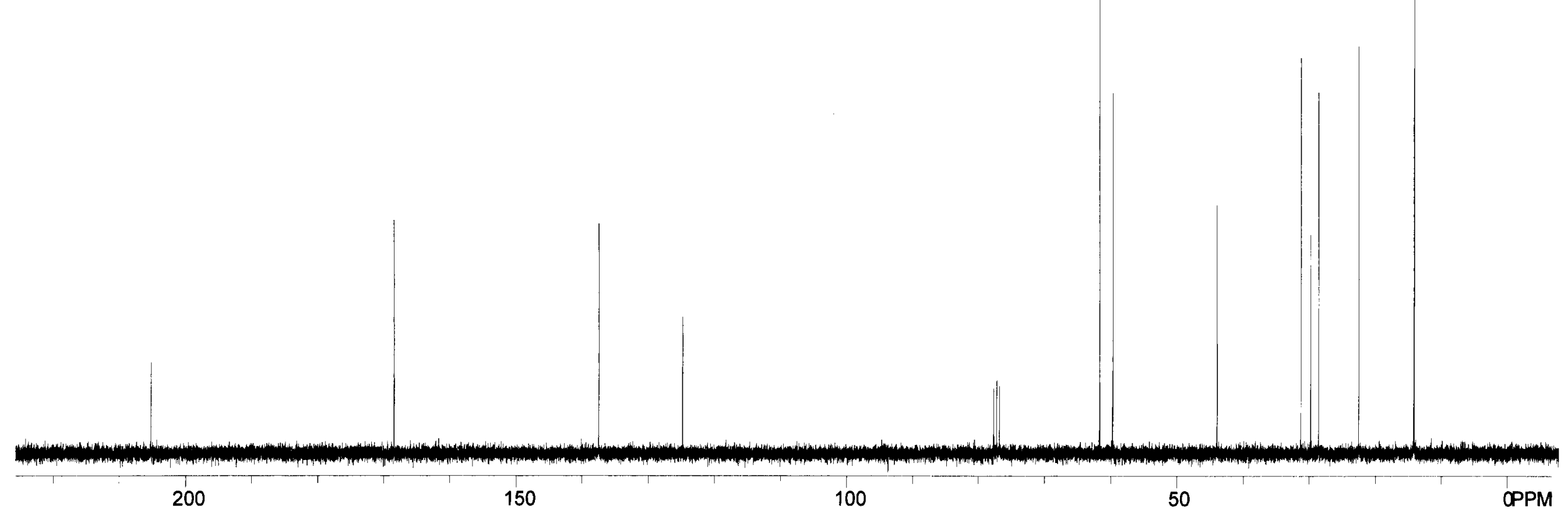




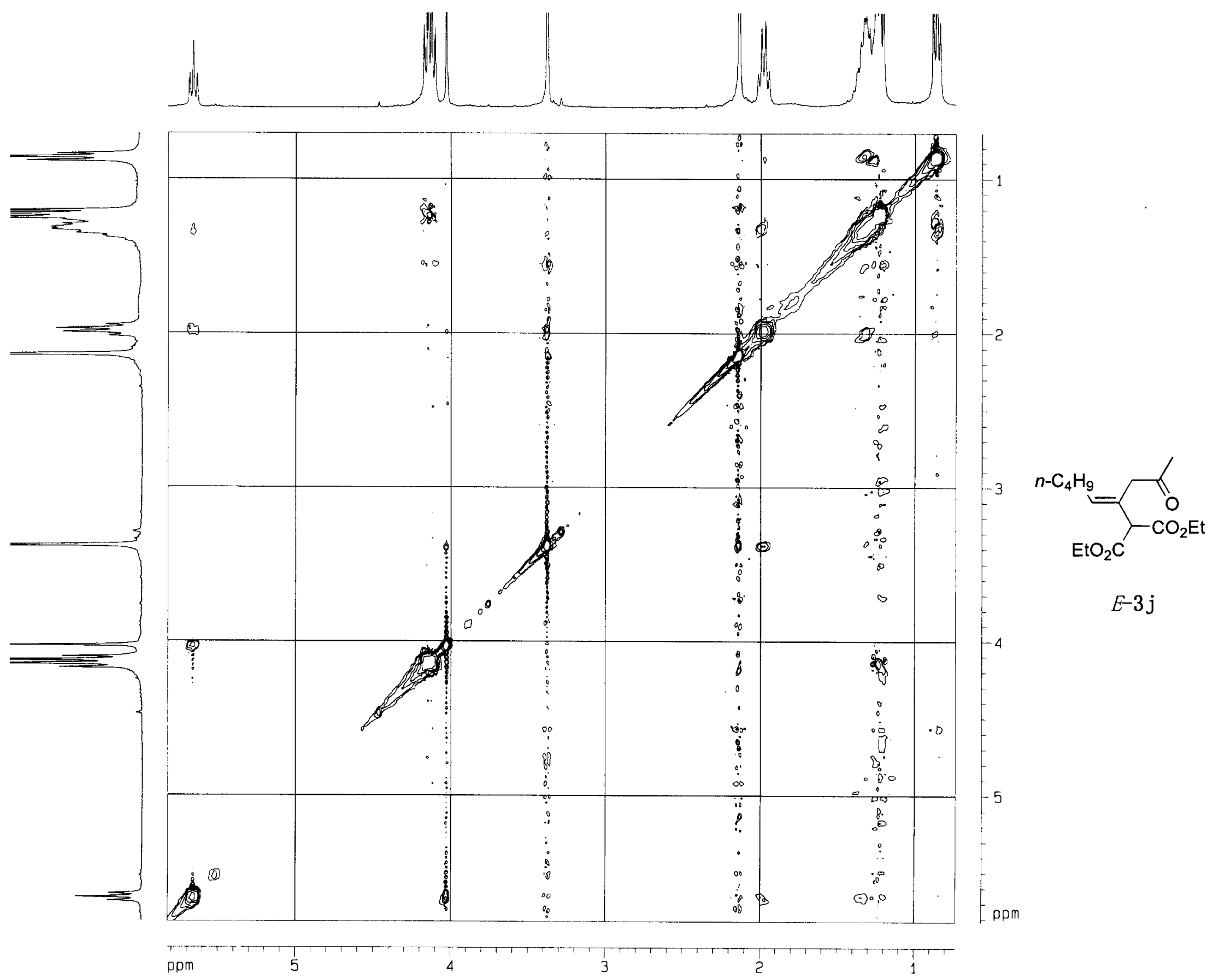




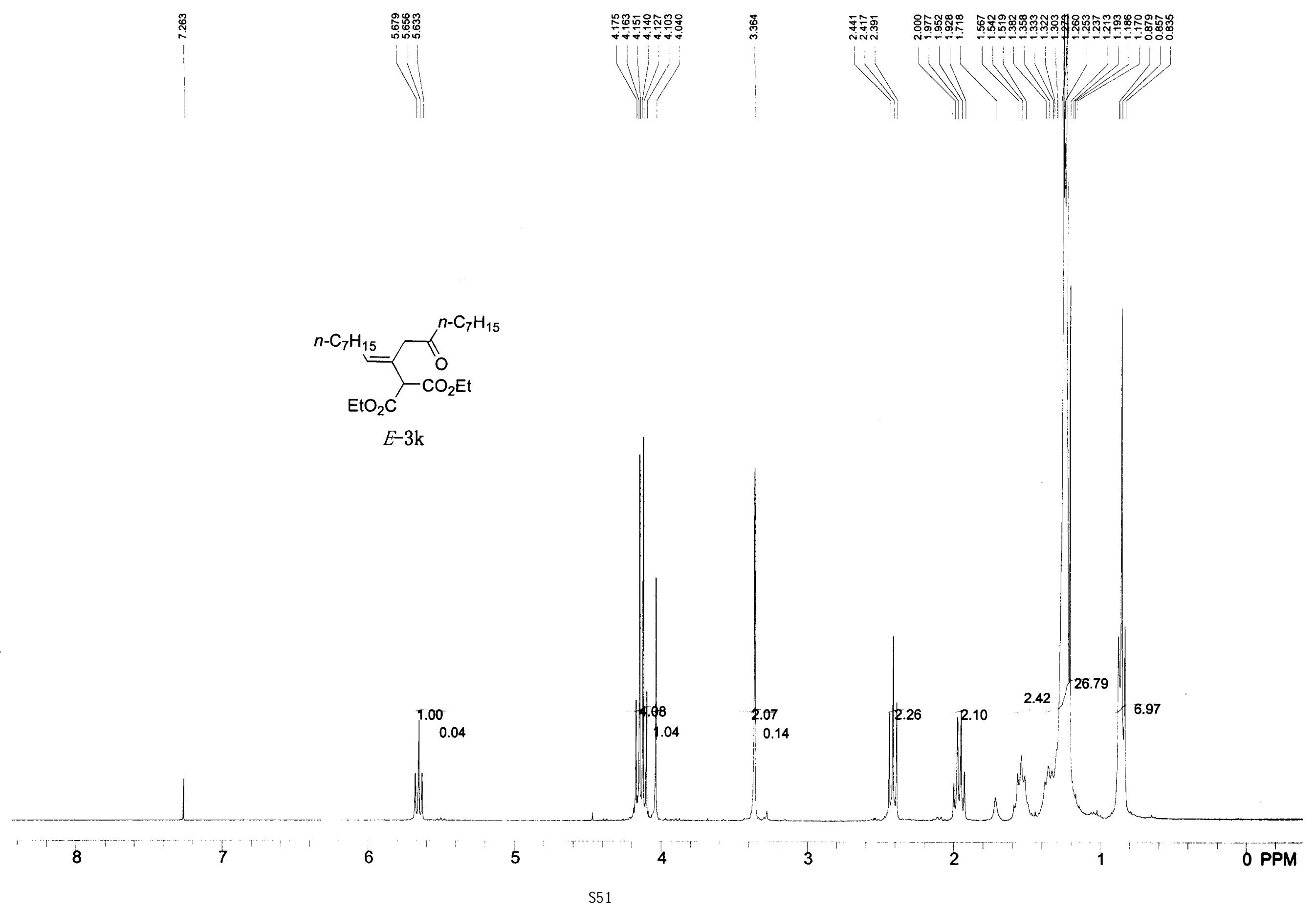



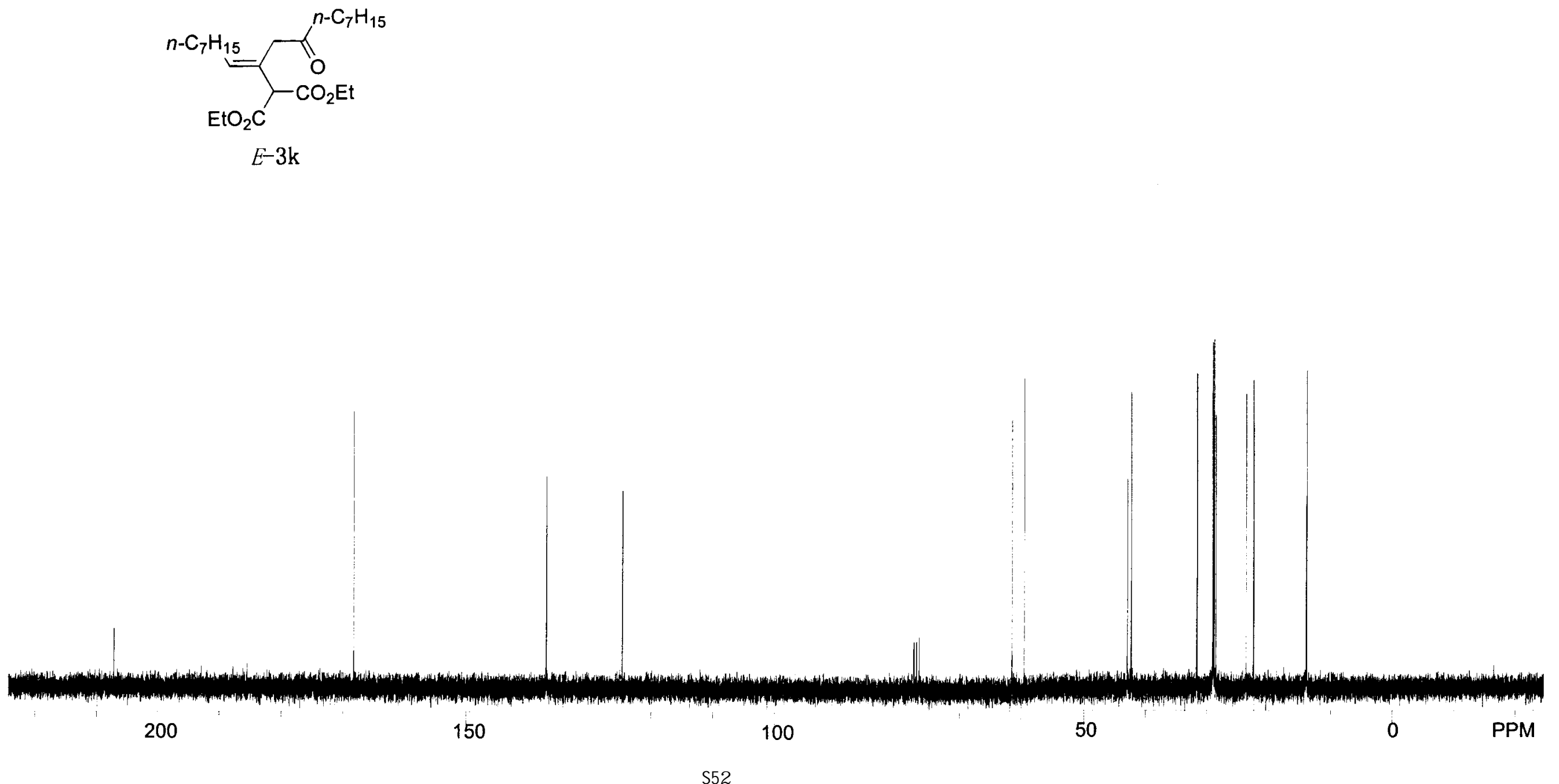

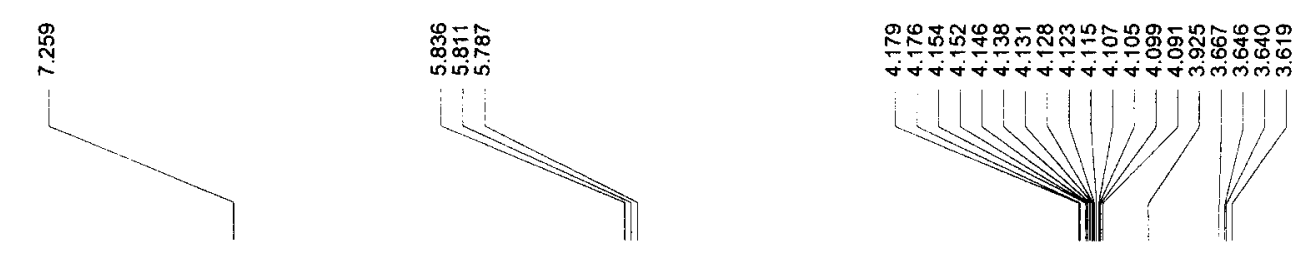

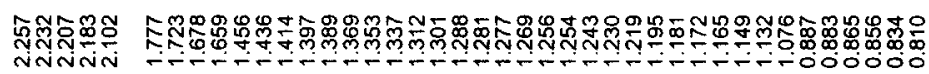
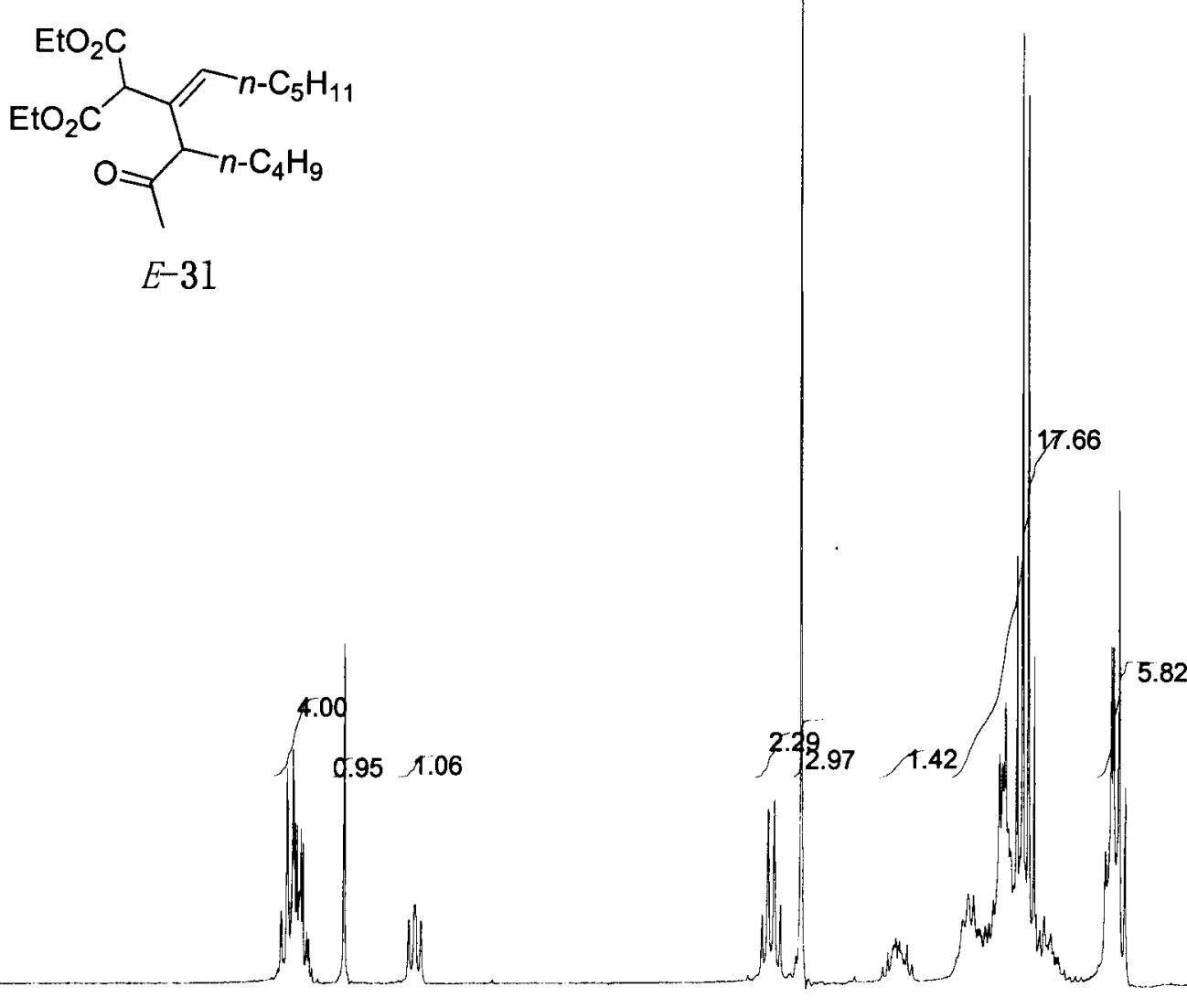
$\mathrm{EtO}_{2} \mathrm{C}$<smiles>CCOC(=O)C(=CC(=O)c1ccccc1)C(C(C)=O)C(C(C)=O)C(=O)OCC</smiles> 
$\mathrm{EtO}_{2} \mathrm{C}$<smiles>CCOC(=O)C(=CC(C)CC)C(C(=O)OCC)C(=O)OCC</smiles>

E-3m

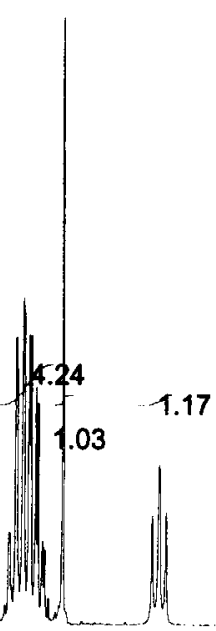
NW N

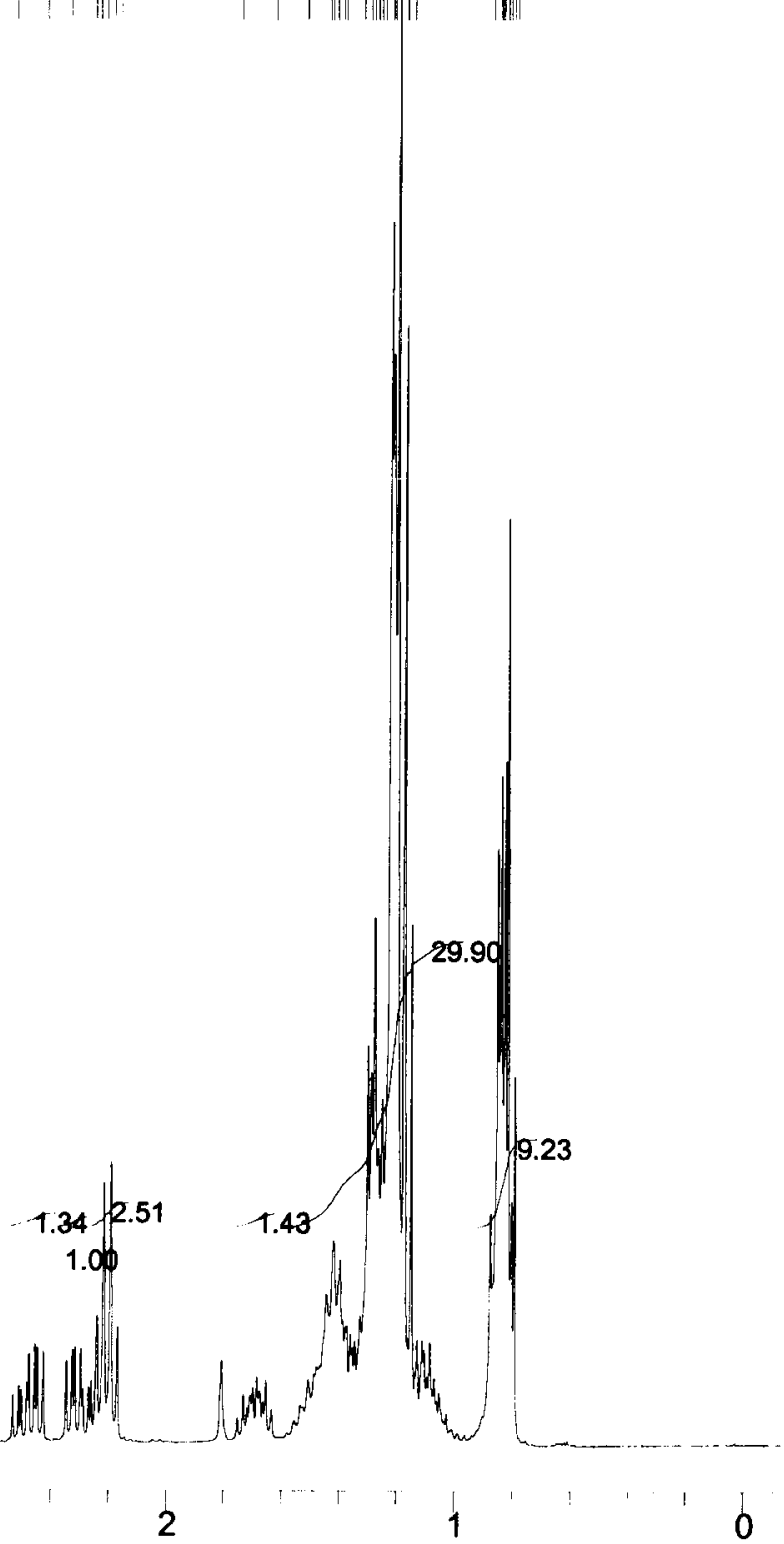




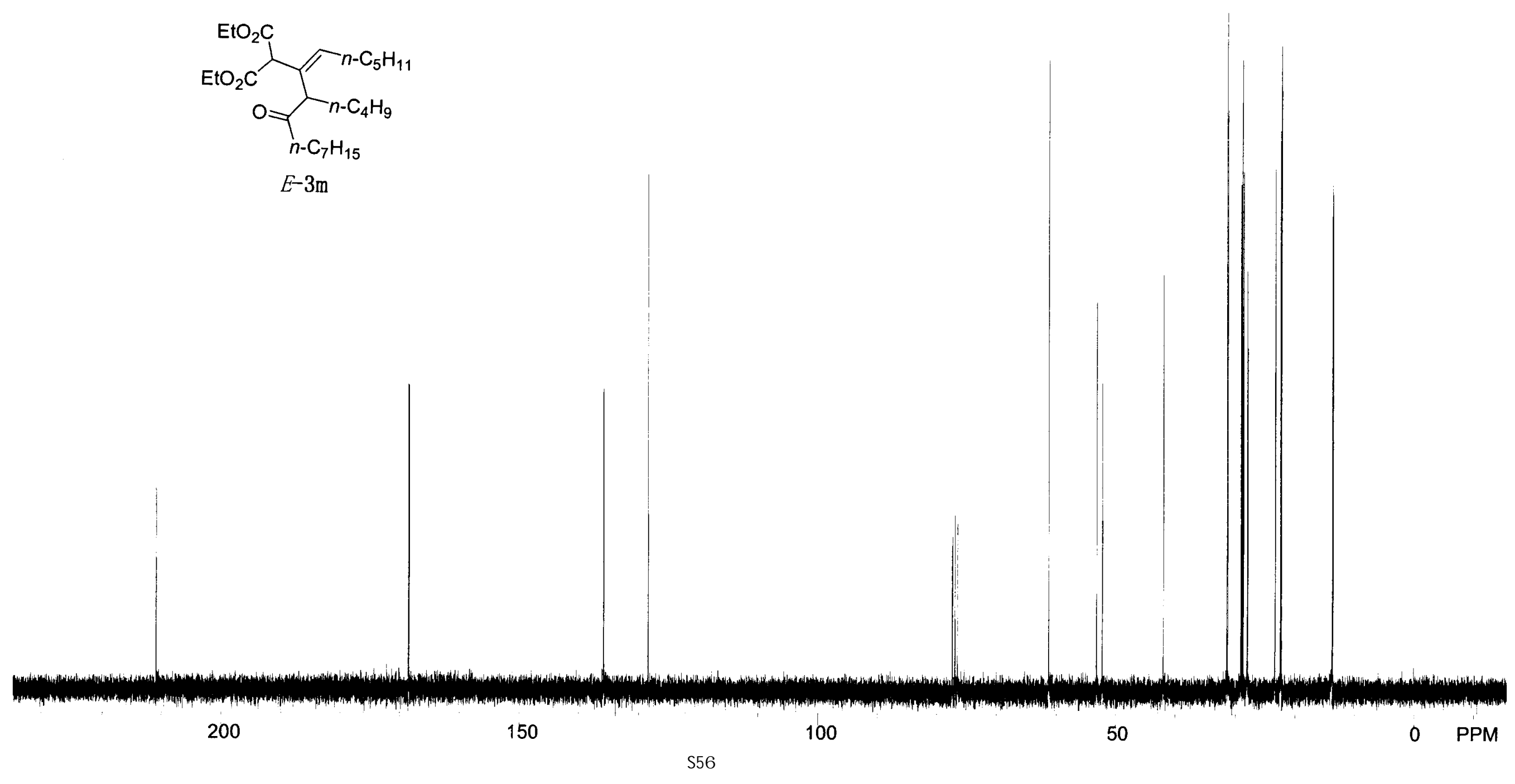

E-3m 

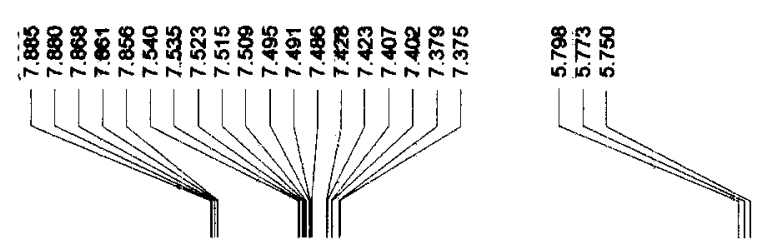

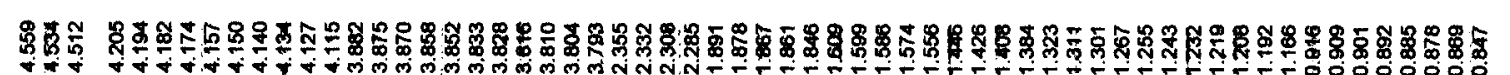

(

E-3n

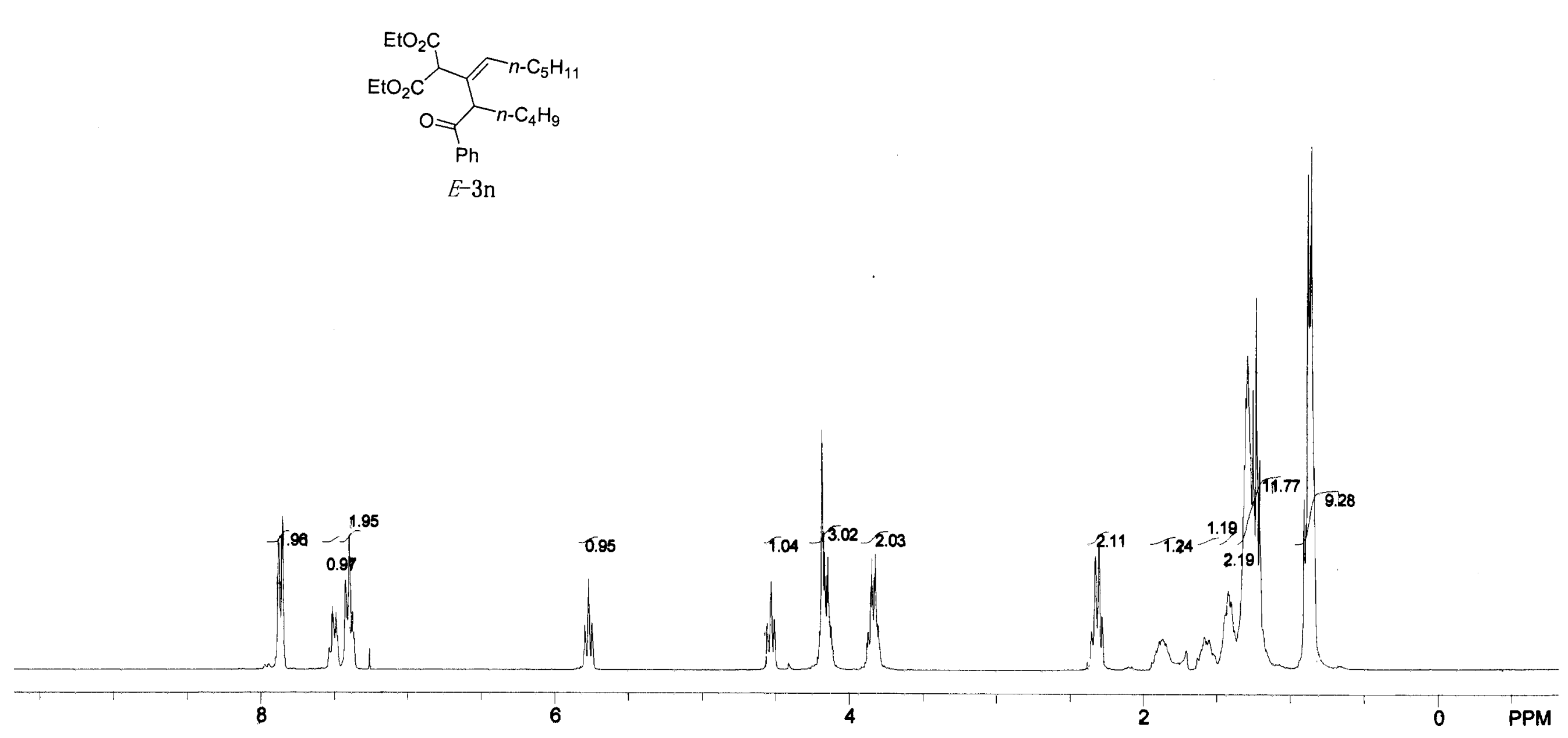



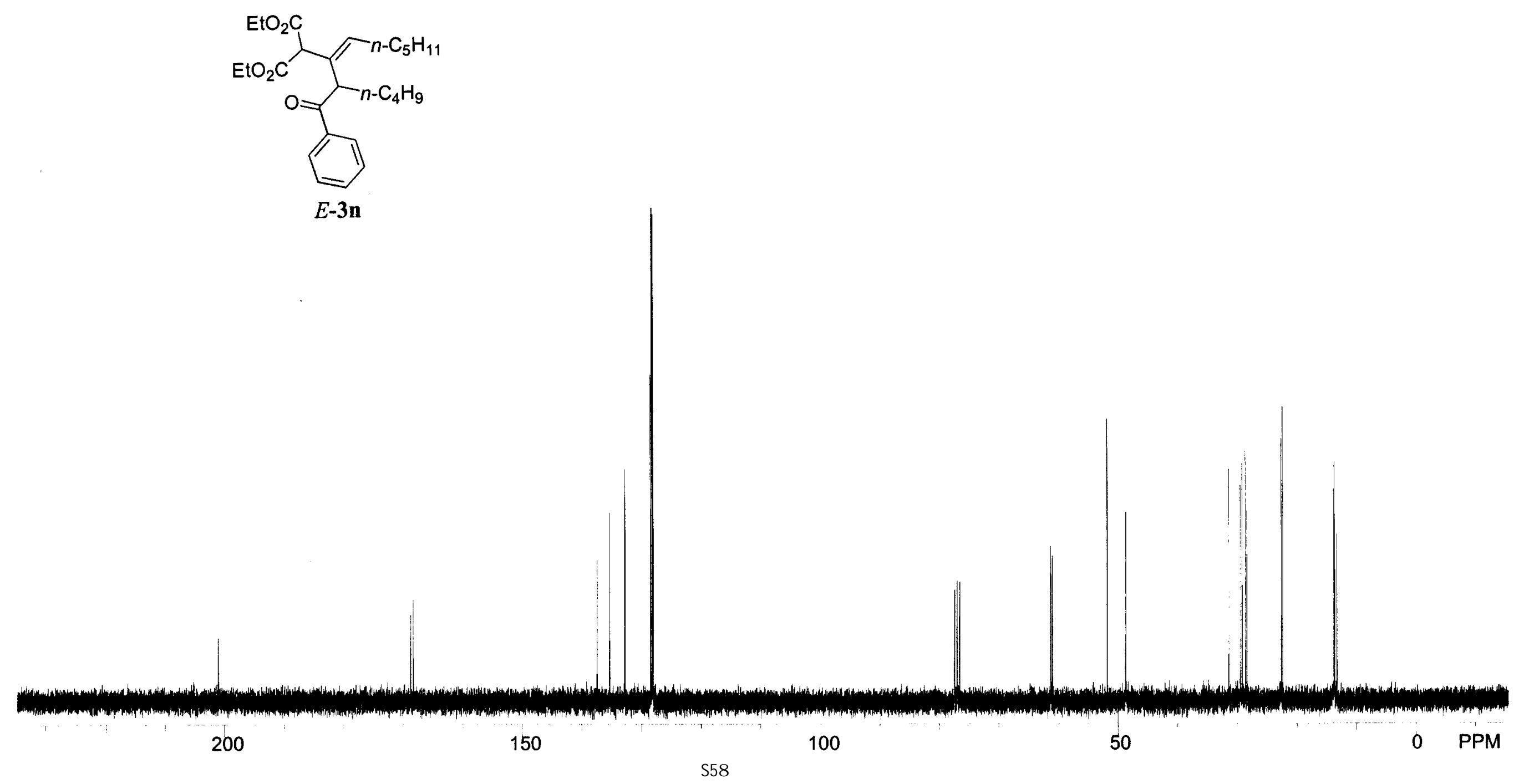

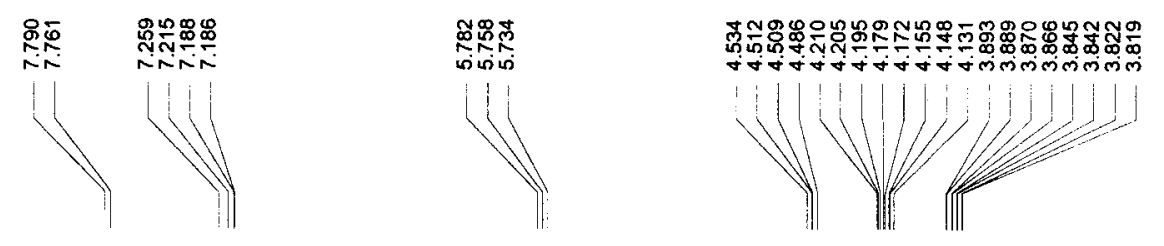

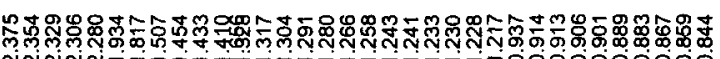
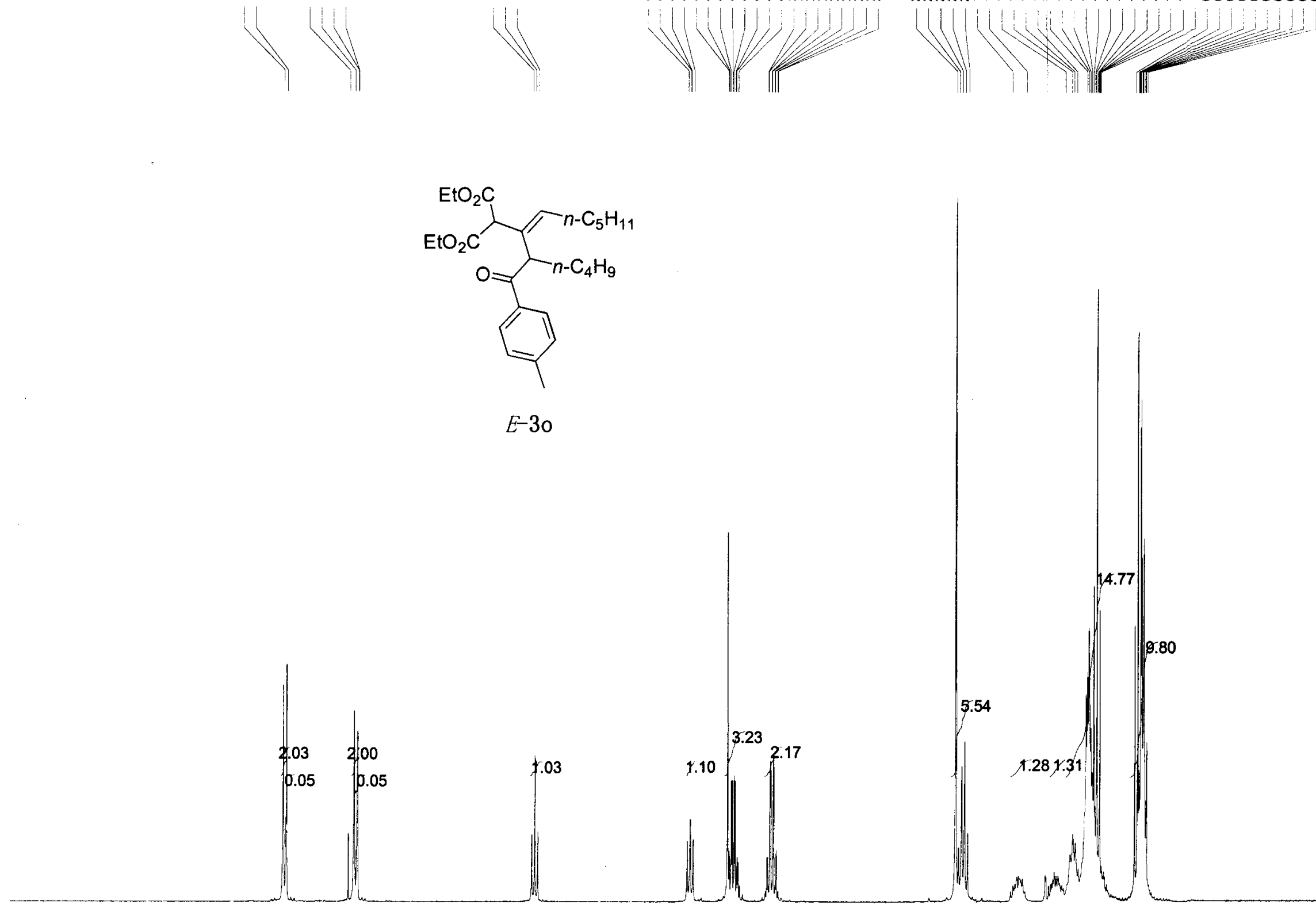

03

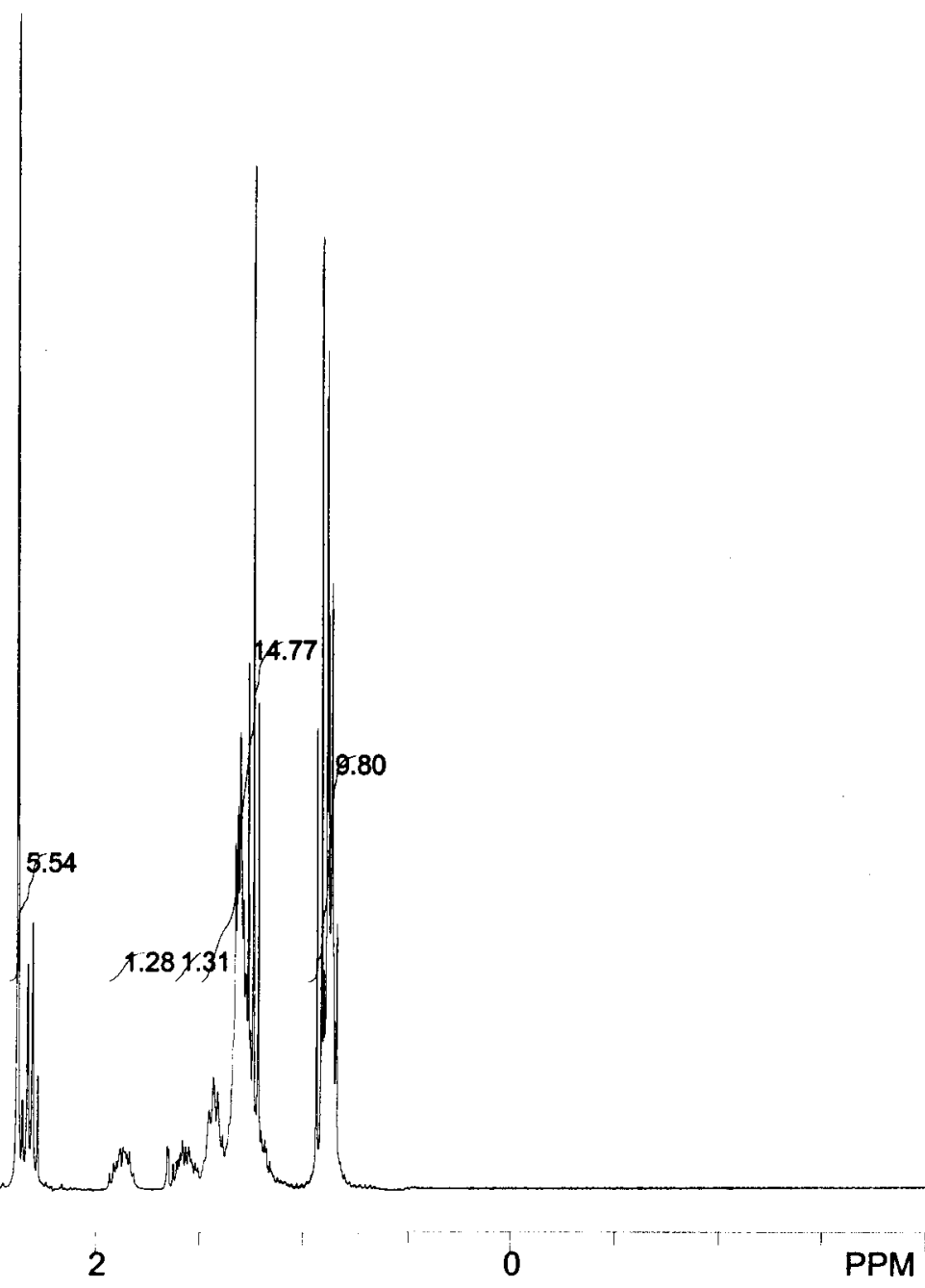




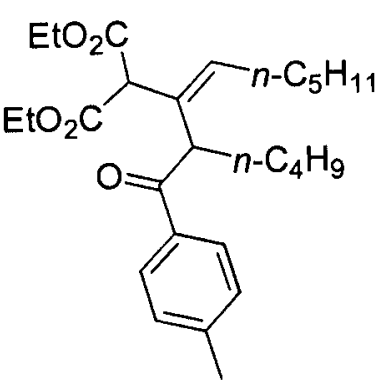

$E-30$ 


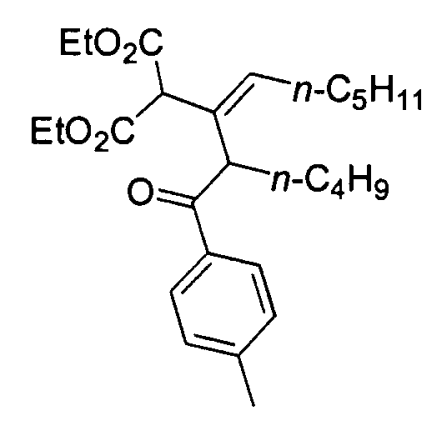

E-3o
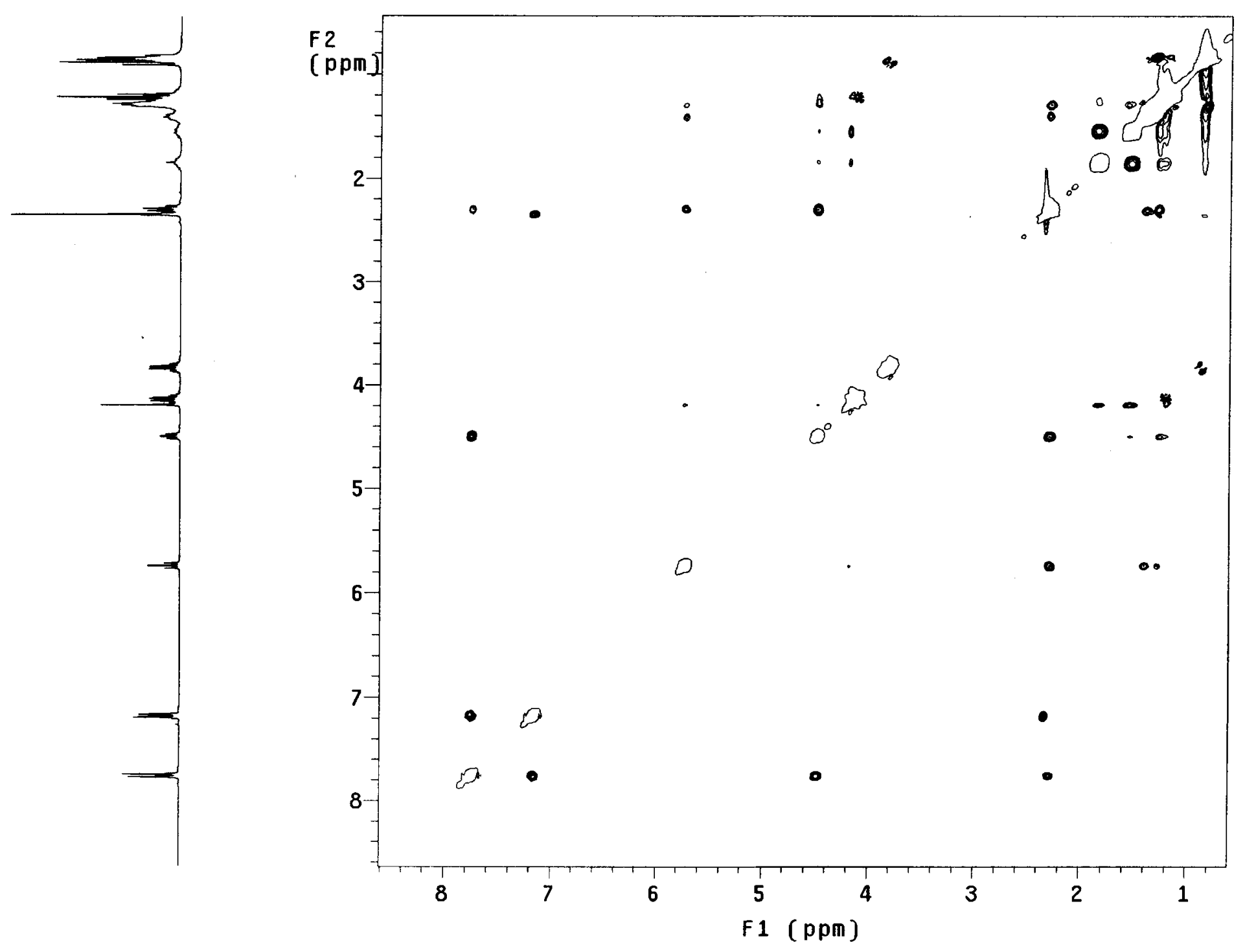

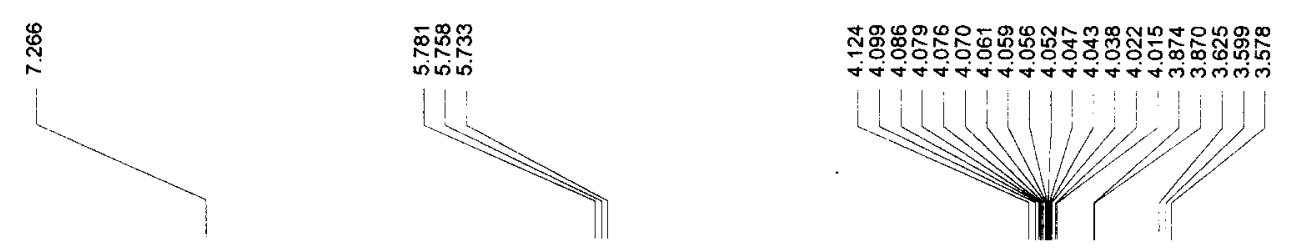

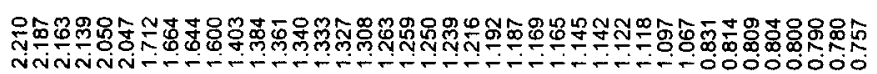
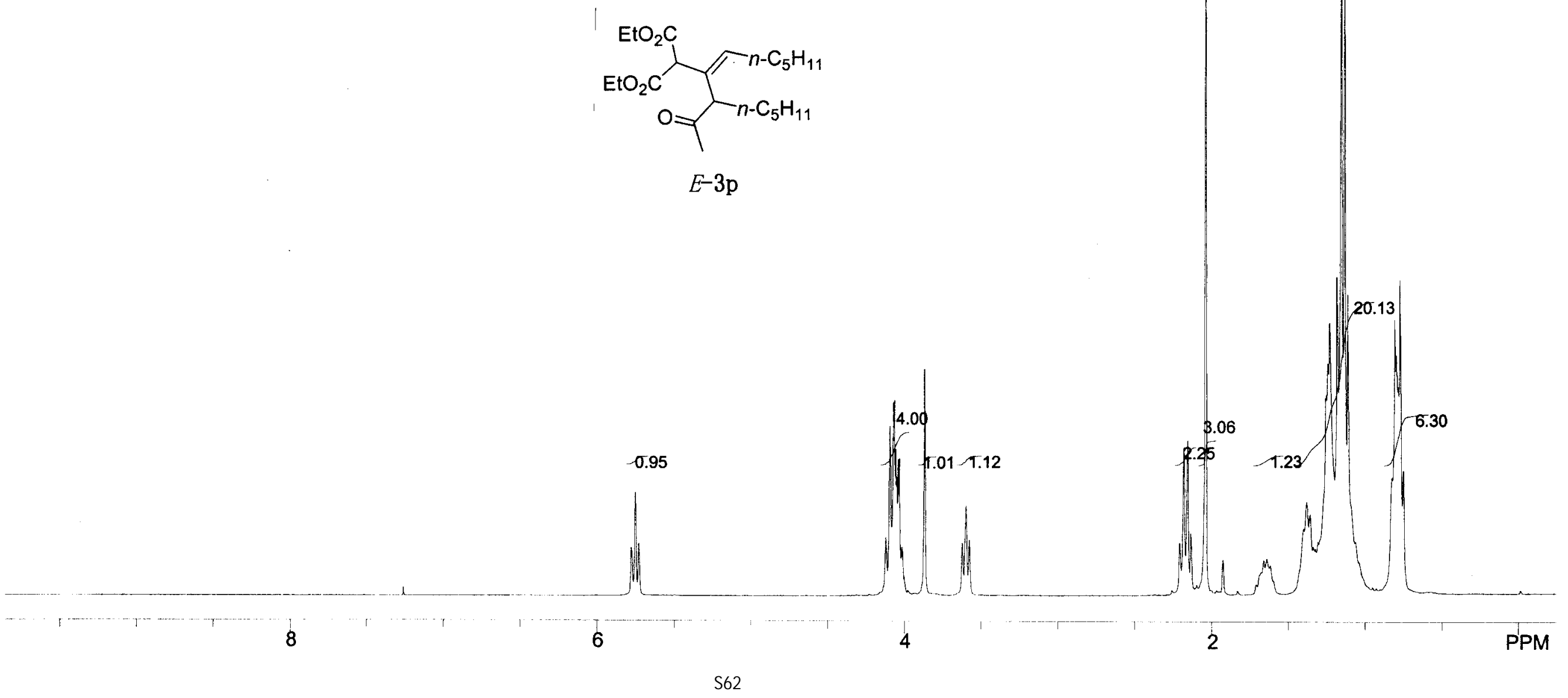


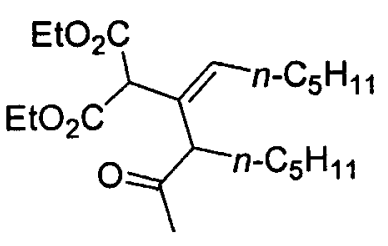

E-3p

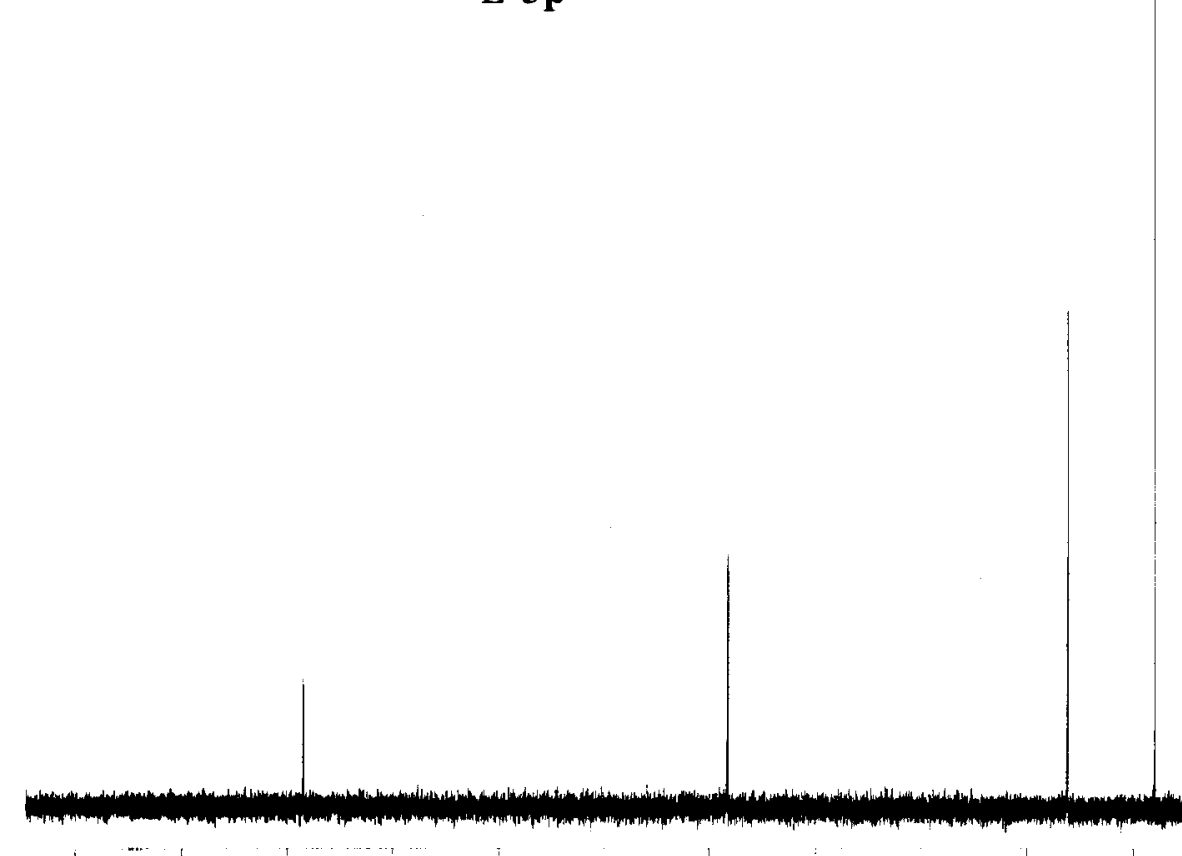




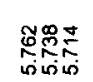

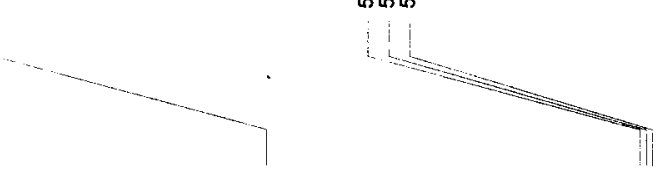

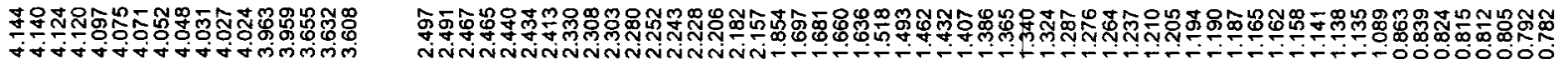

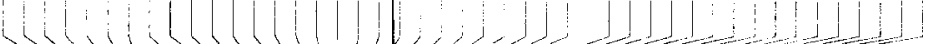

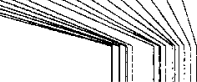
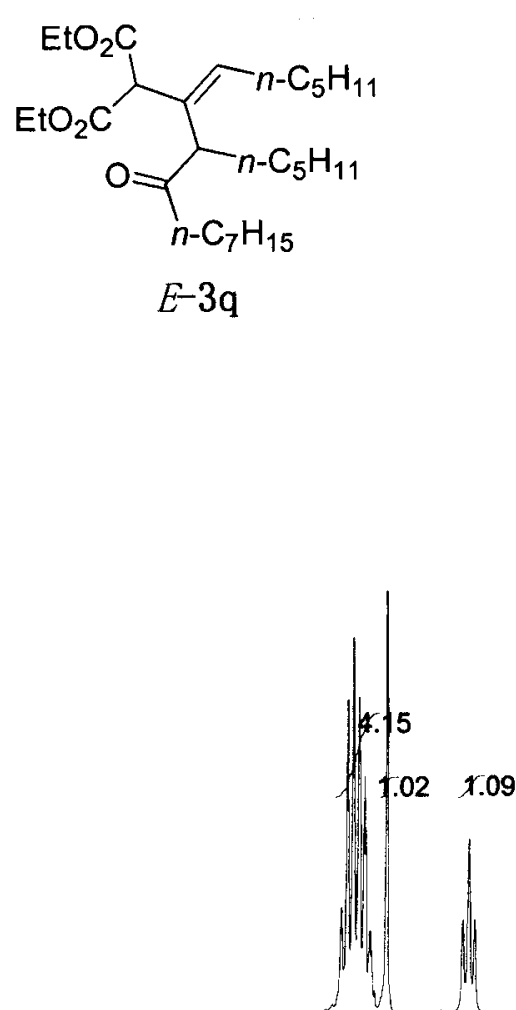

4

31.14

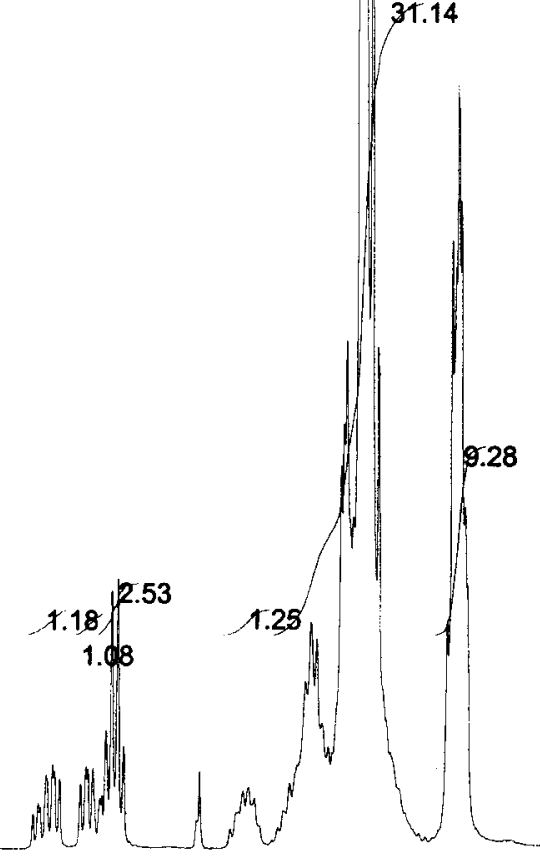

PPM 


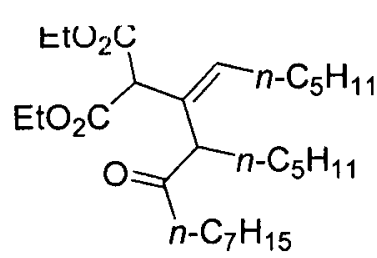

E-3q

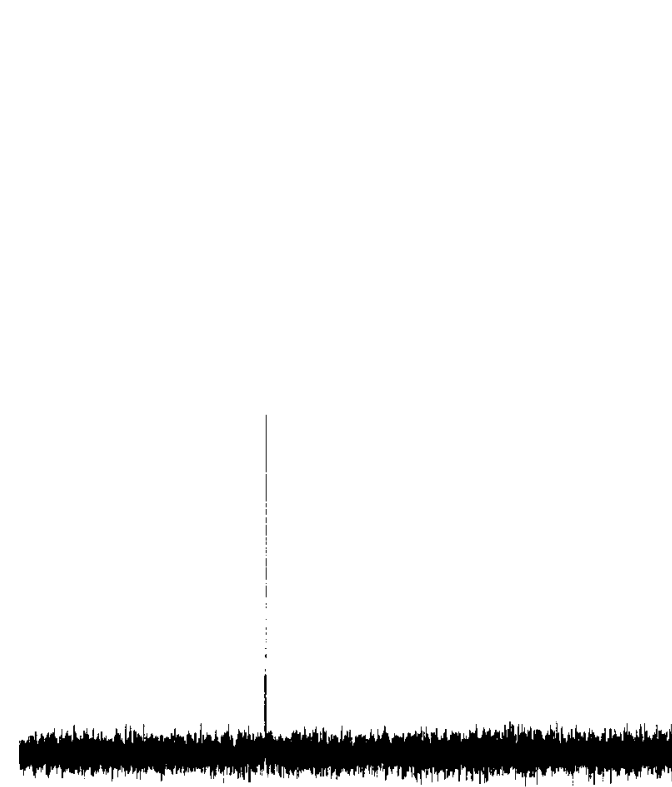




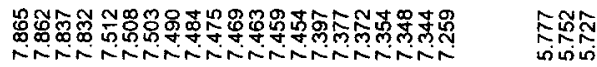

LLLLLi(l) LU)

$\rightarrow$

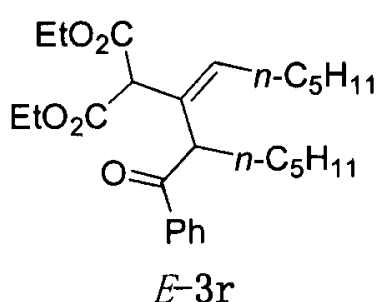

$E-3 \mathrm{r}$
勇福

$1)]$ ] [L]

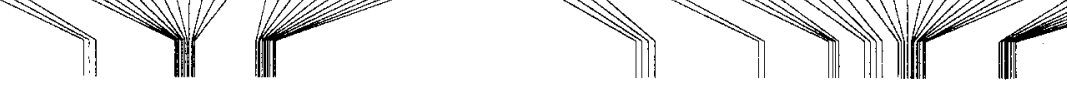



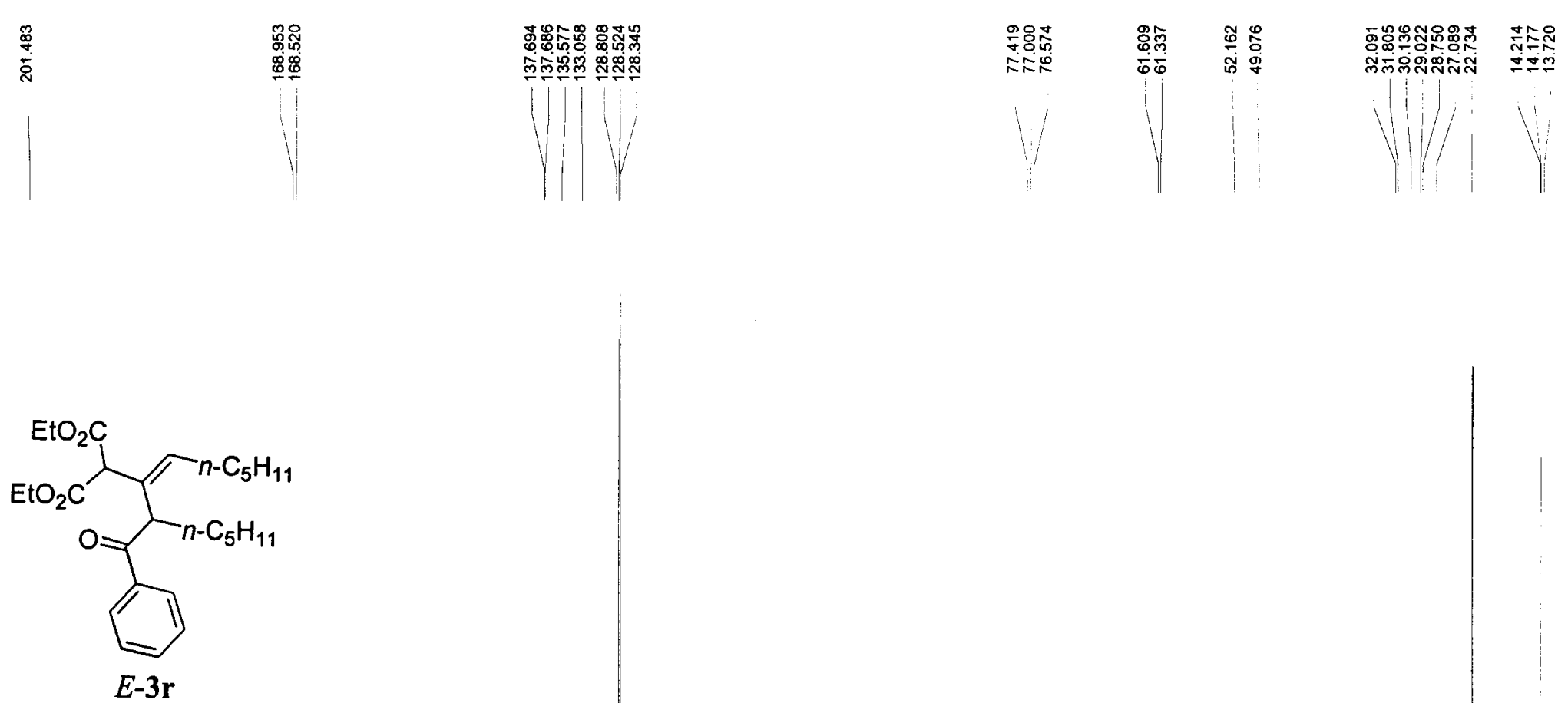


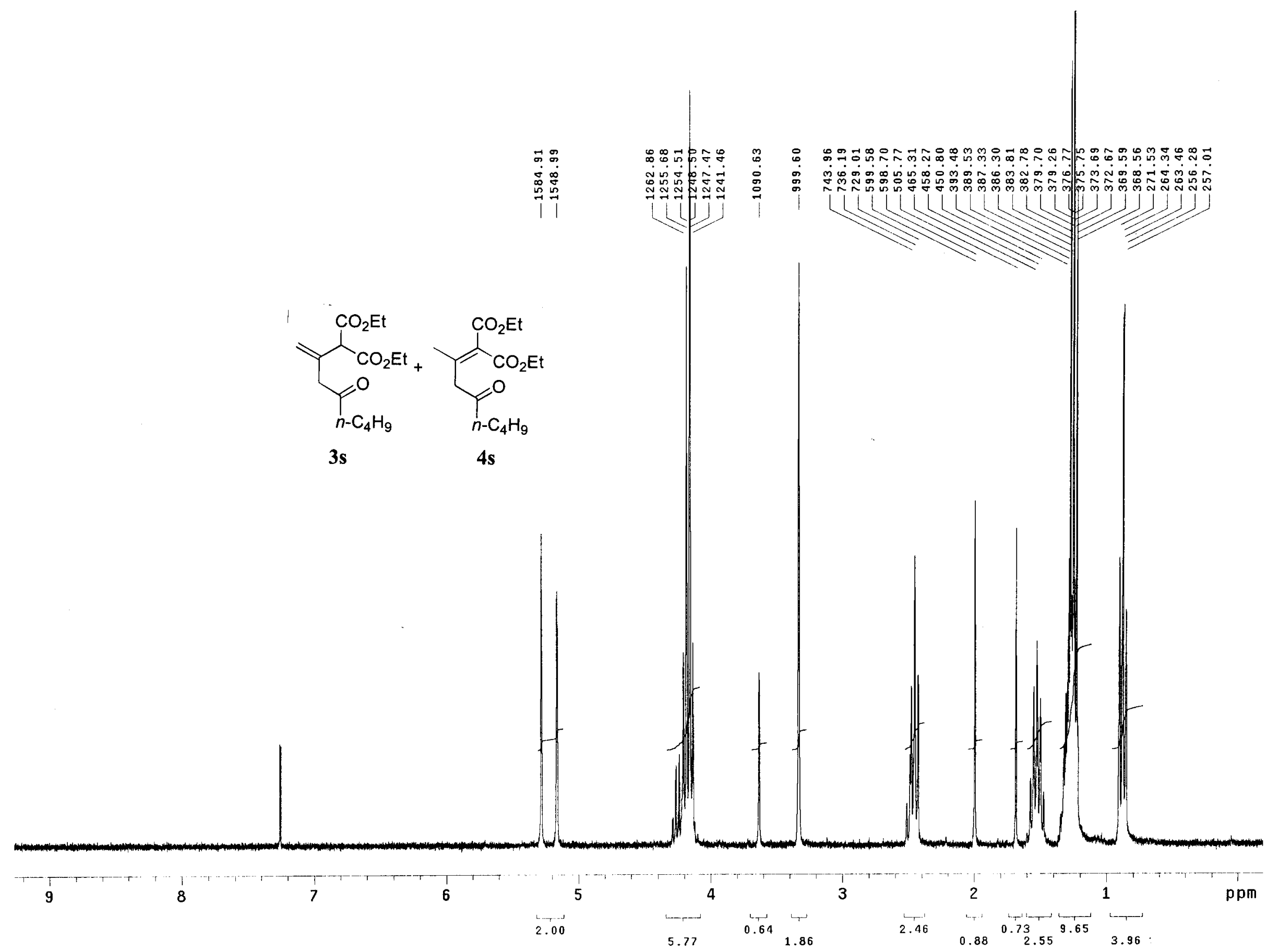




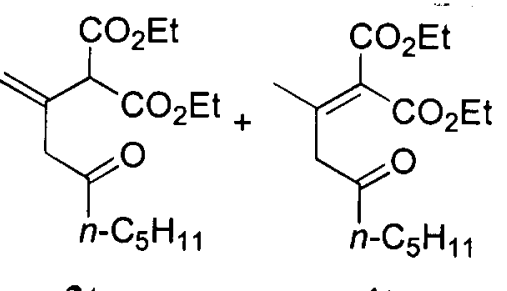

$3 t$
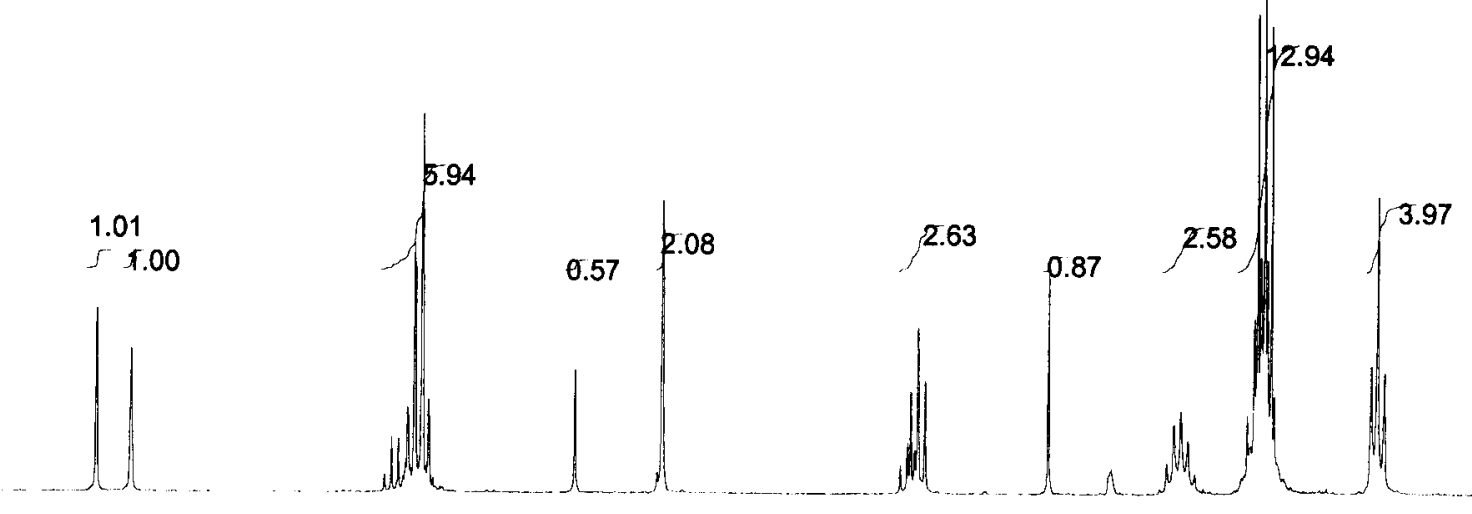

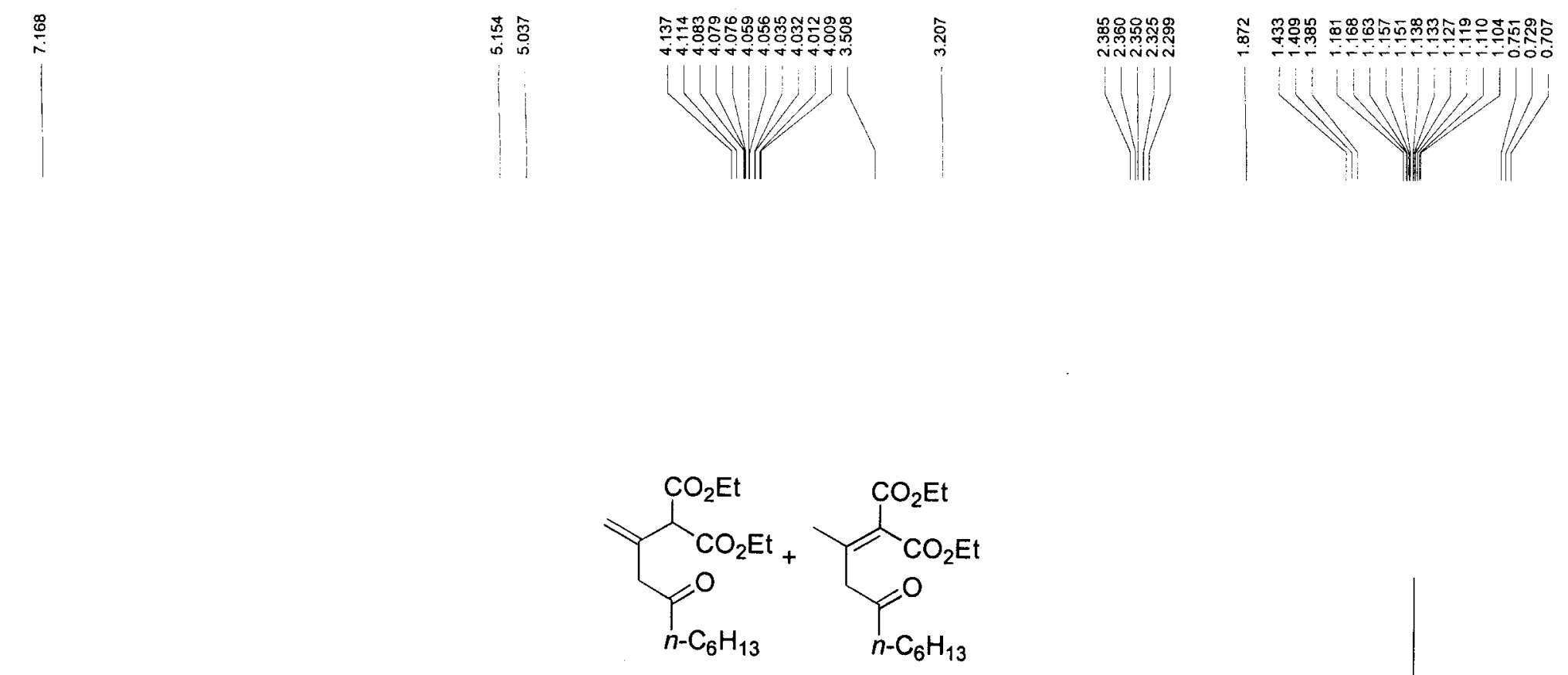

3u

$4 u$

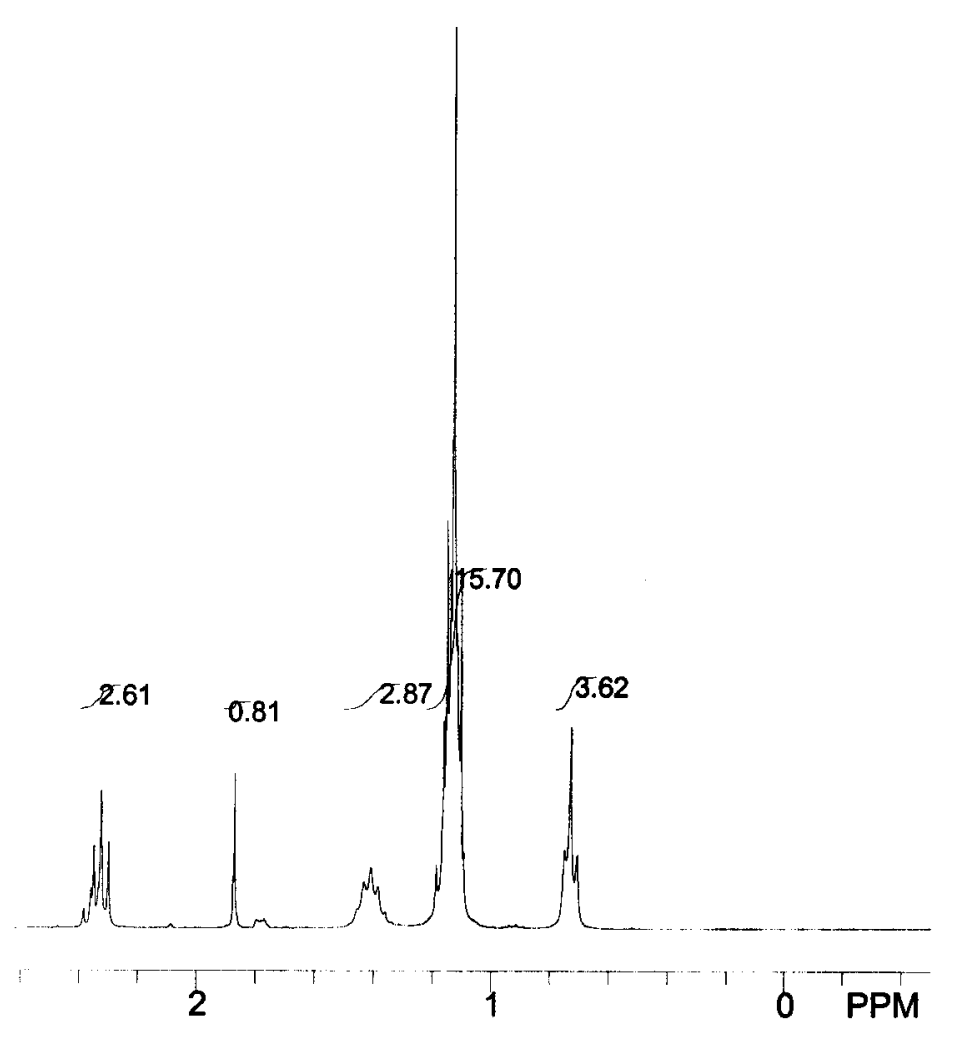




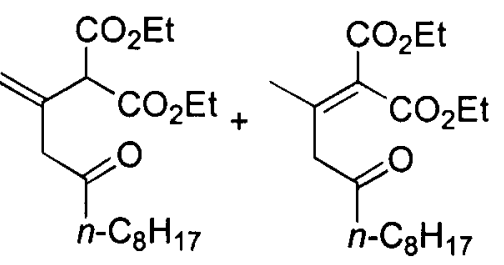

3v

$4 v$
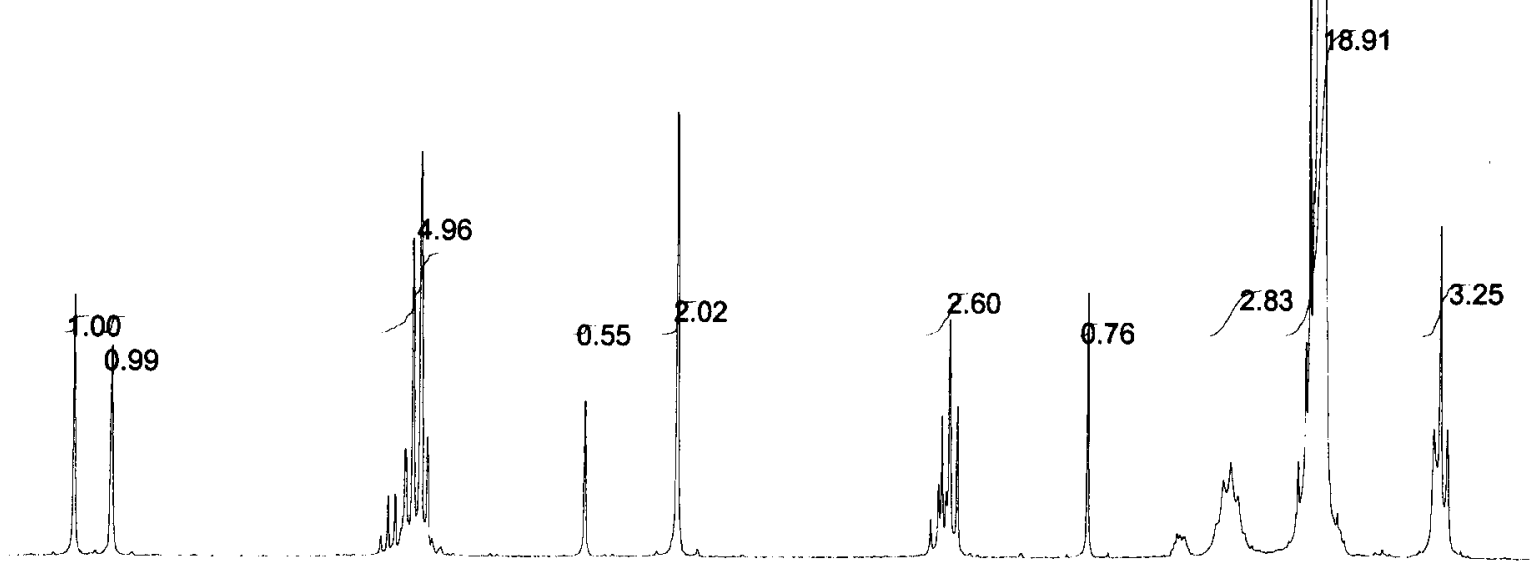
जN
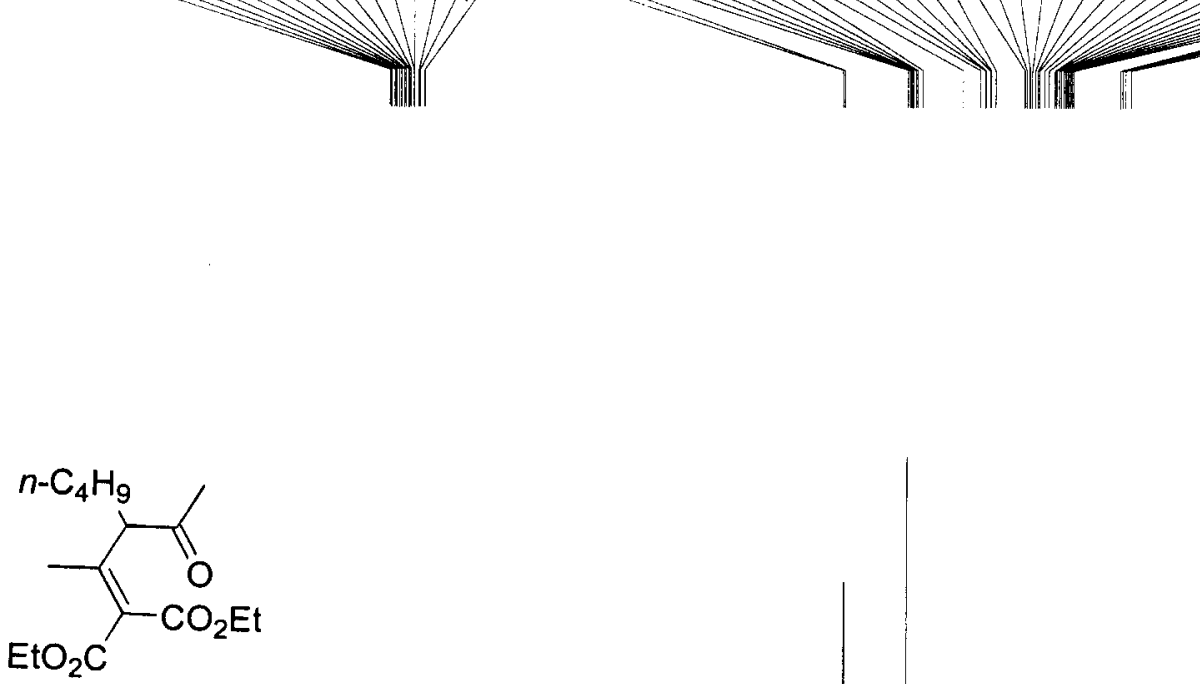

$4 \mathrm{a}$ 
Pulse 92.9 degrees

Acq. time $2.000 \mathrm{sec}$

180 repeditions

OBSERVE \&13, 75.4420774 MHZ

POUPLE H1, 300.0294698 MHZ

POwer 48 dB,

Cont inuoys iy on

DATA PROCESSING

Line broadening $1.0 \mathrm{~Hz}$

Fotal time $1 \mathrm{hr}, 12 \mathrm{~m} / \mathrm{n}, 49 \mathrm{sec}$
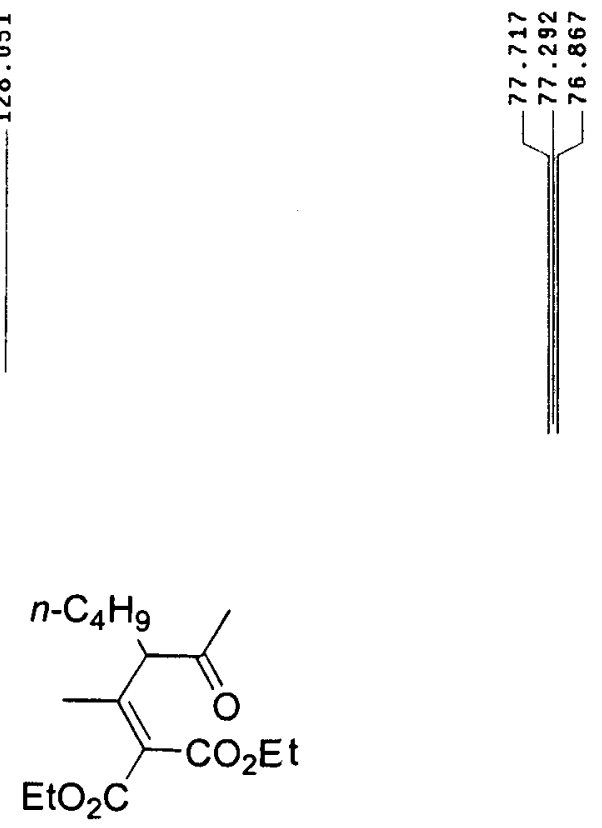

$4 a$
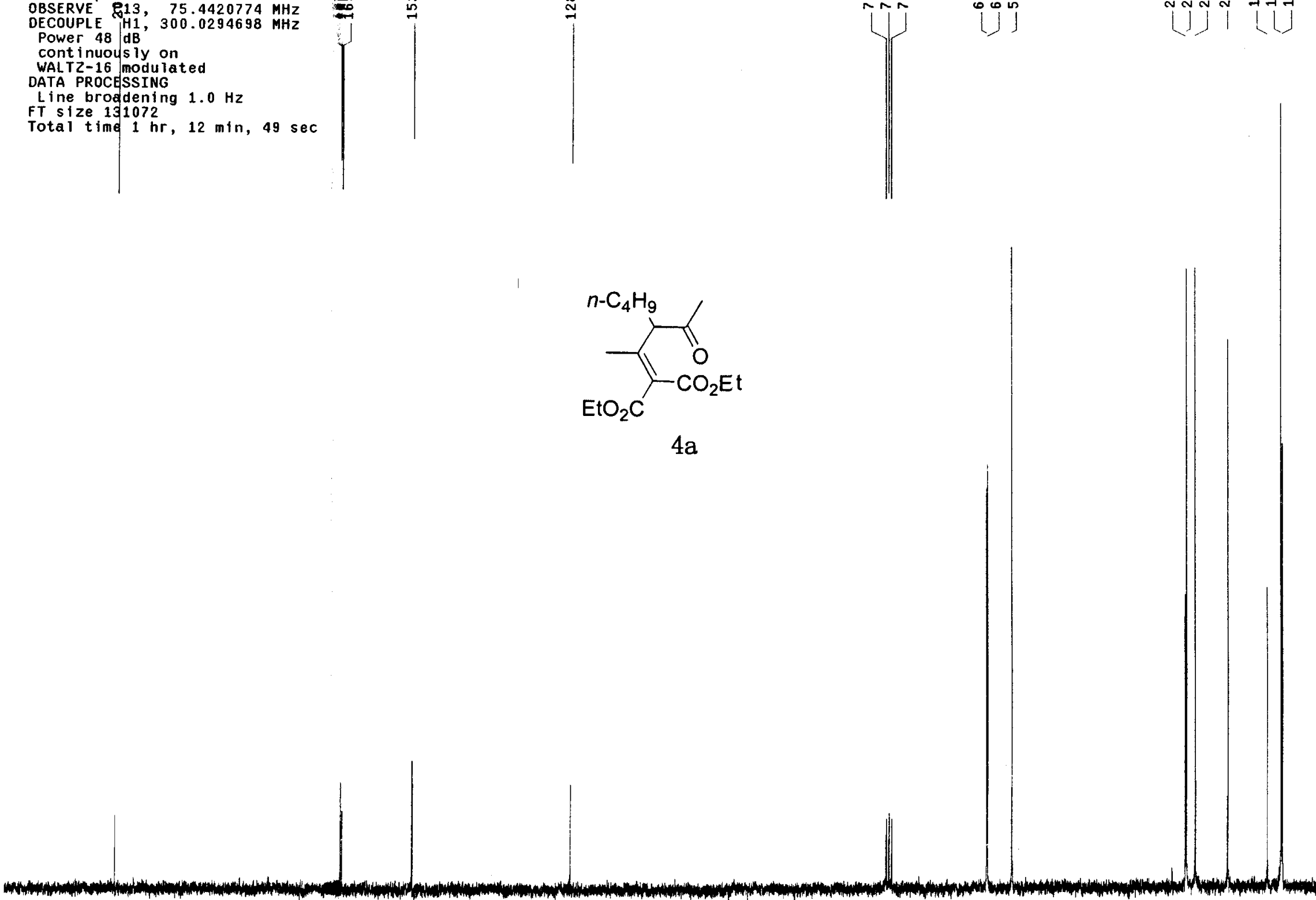


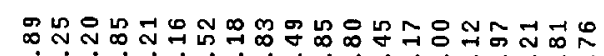

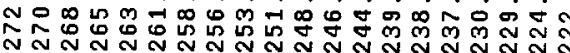

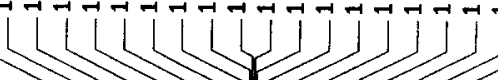

-

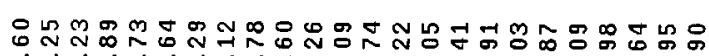

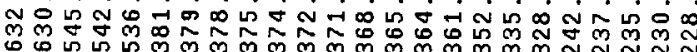

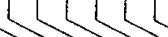

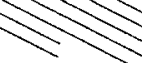

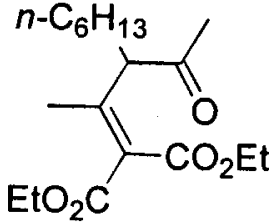

$4 c$

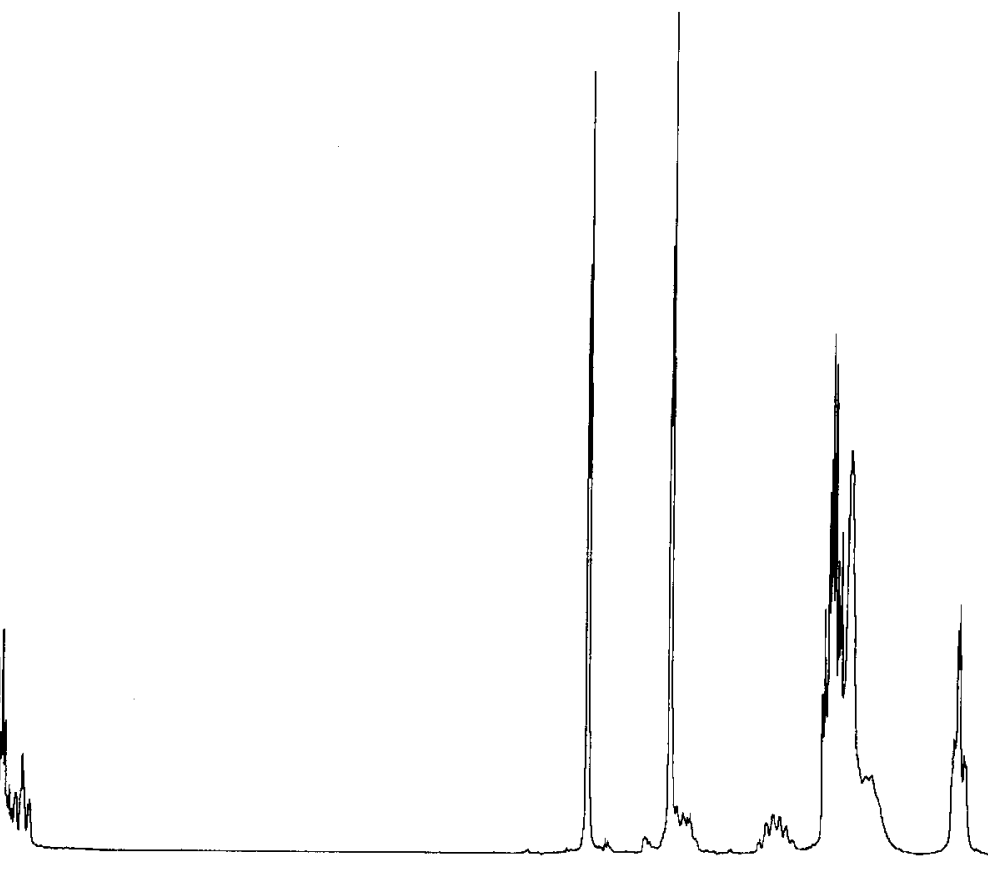
4

2 2.87 4.1 $\frac{1}{4.28}$ 


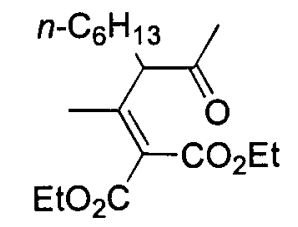

$4 c$

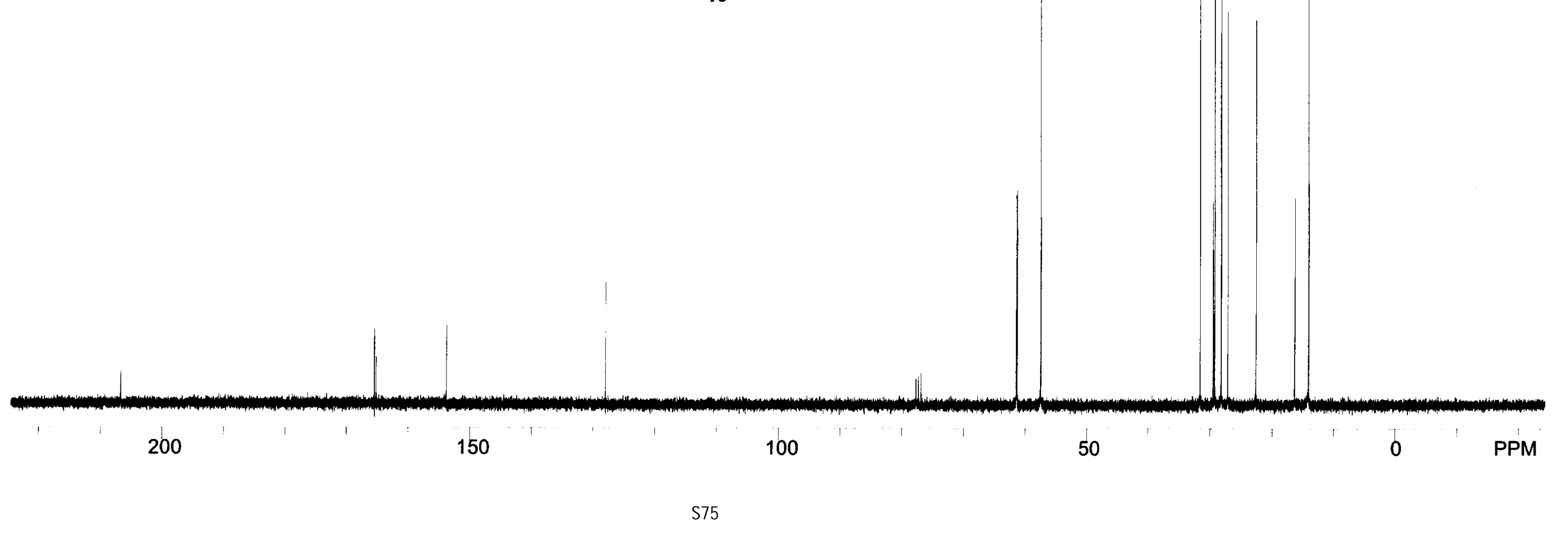




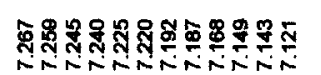

Hiñ

UII

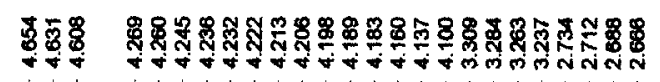

u uWu
5.00

$4 e$
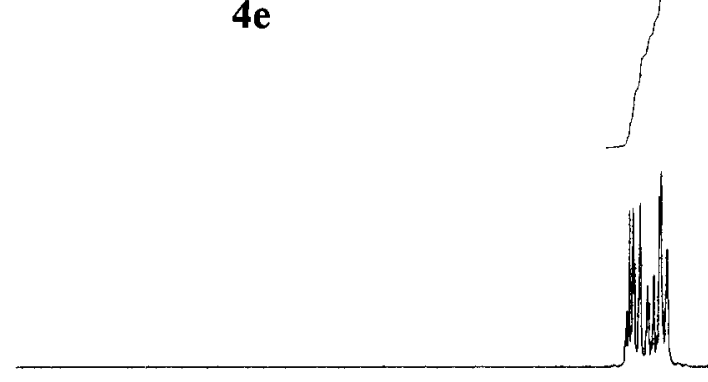

9
8
3.99

$\oint .93$

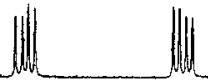

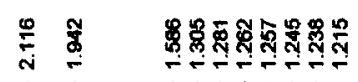

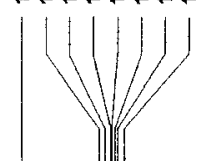

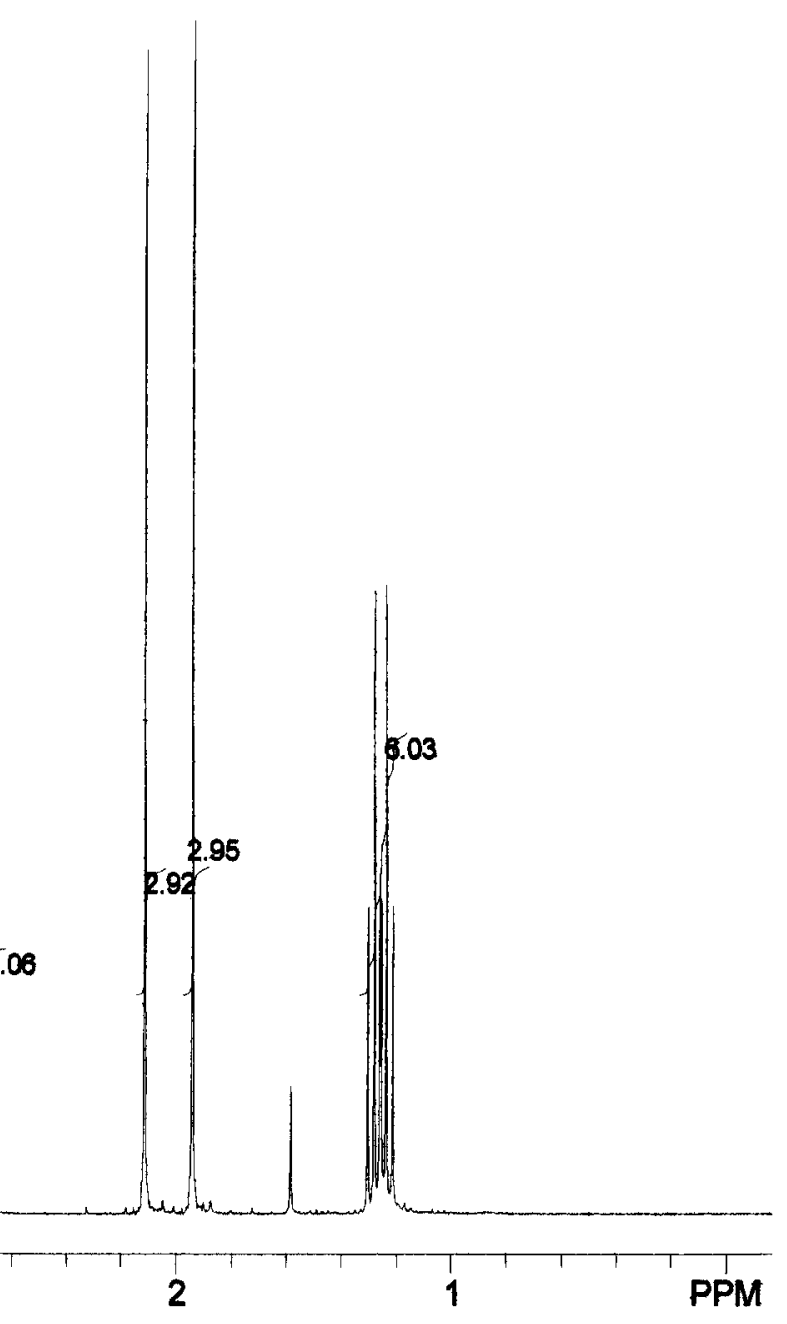




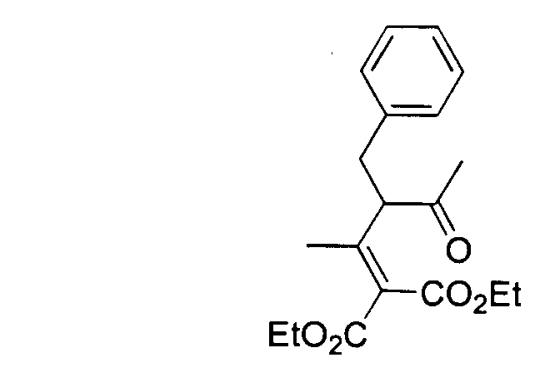

$4 e$

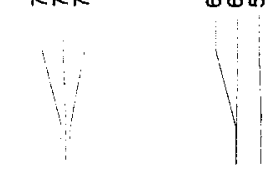

$+1$

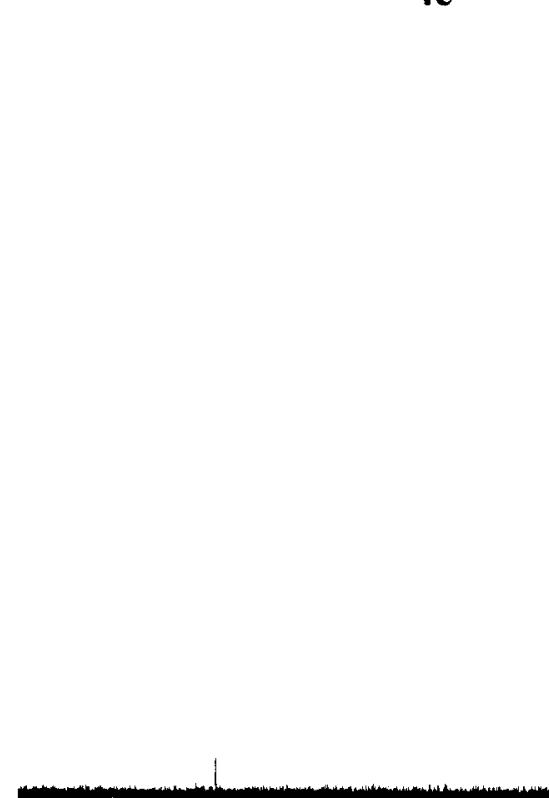

200 


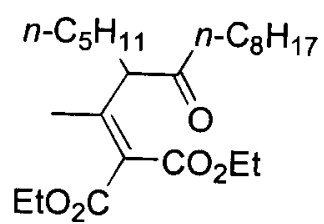

$4 f$

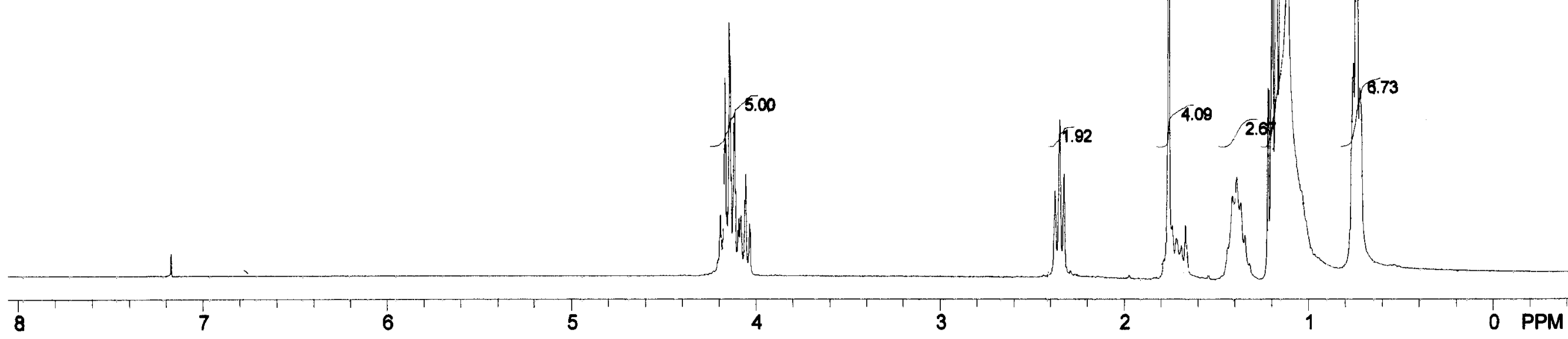




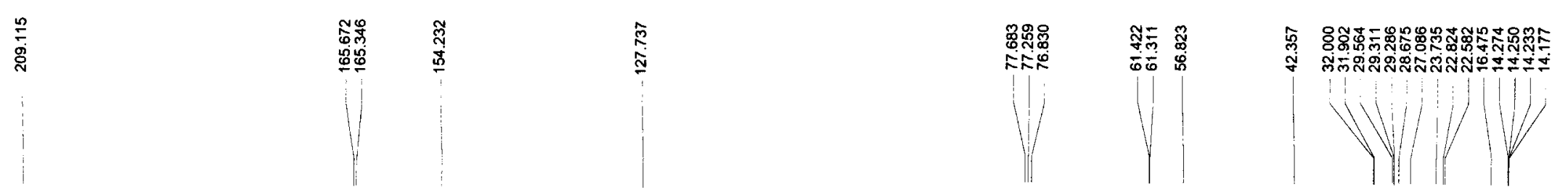
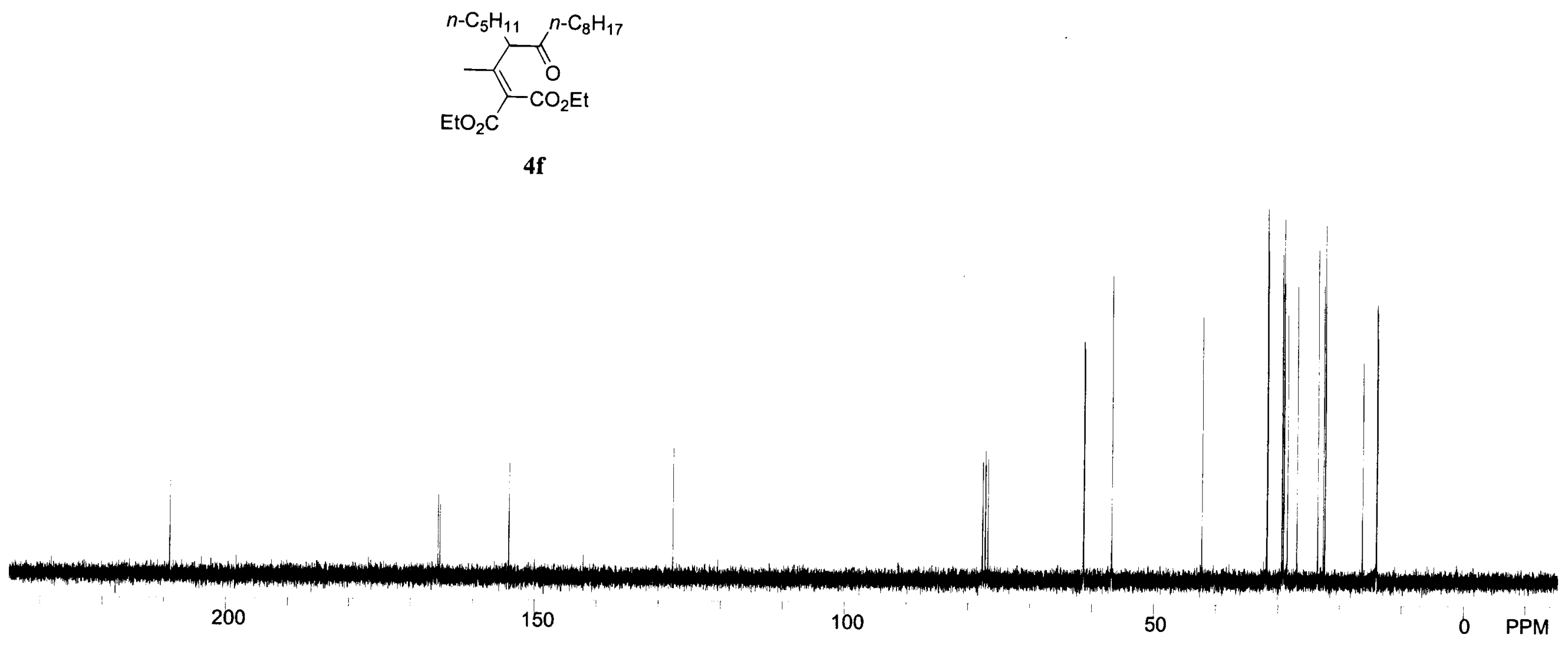


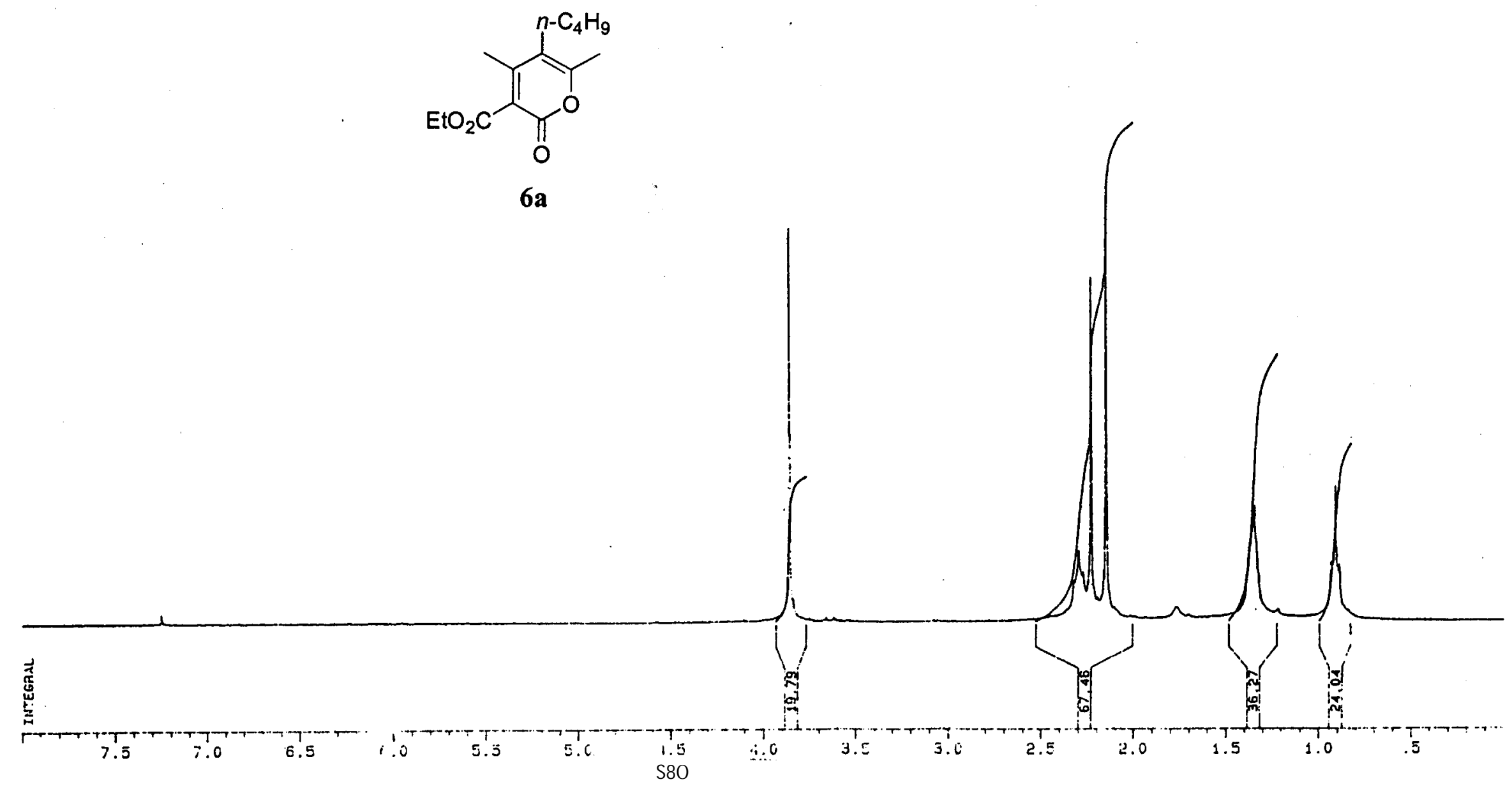




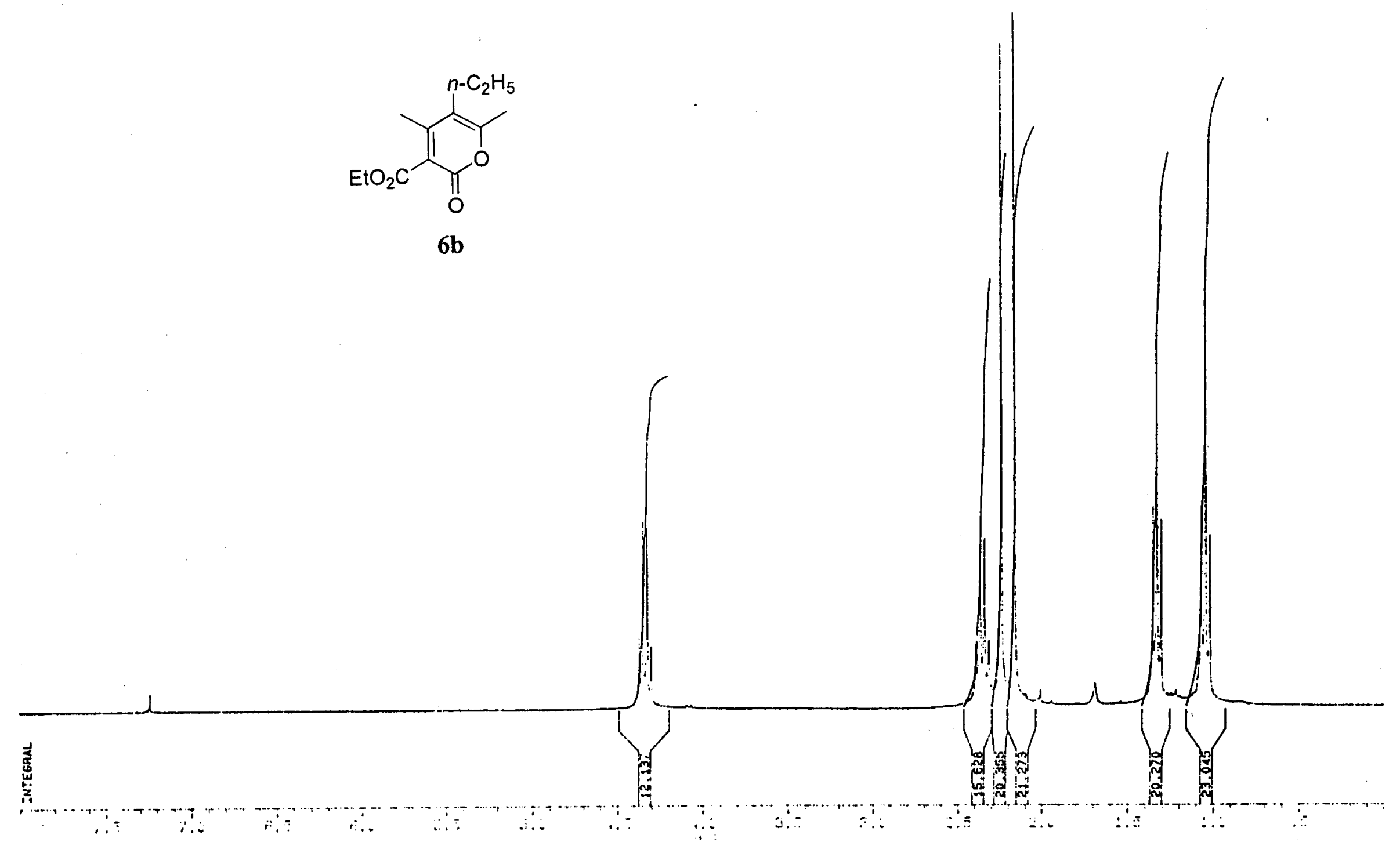



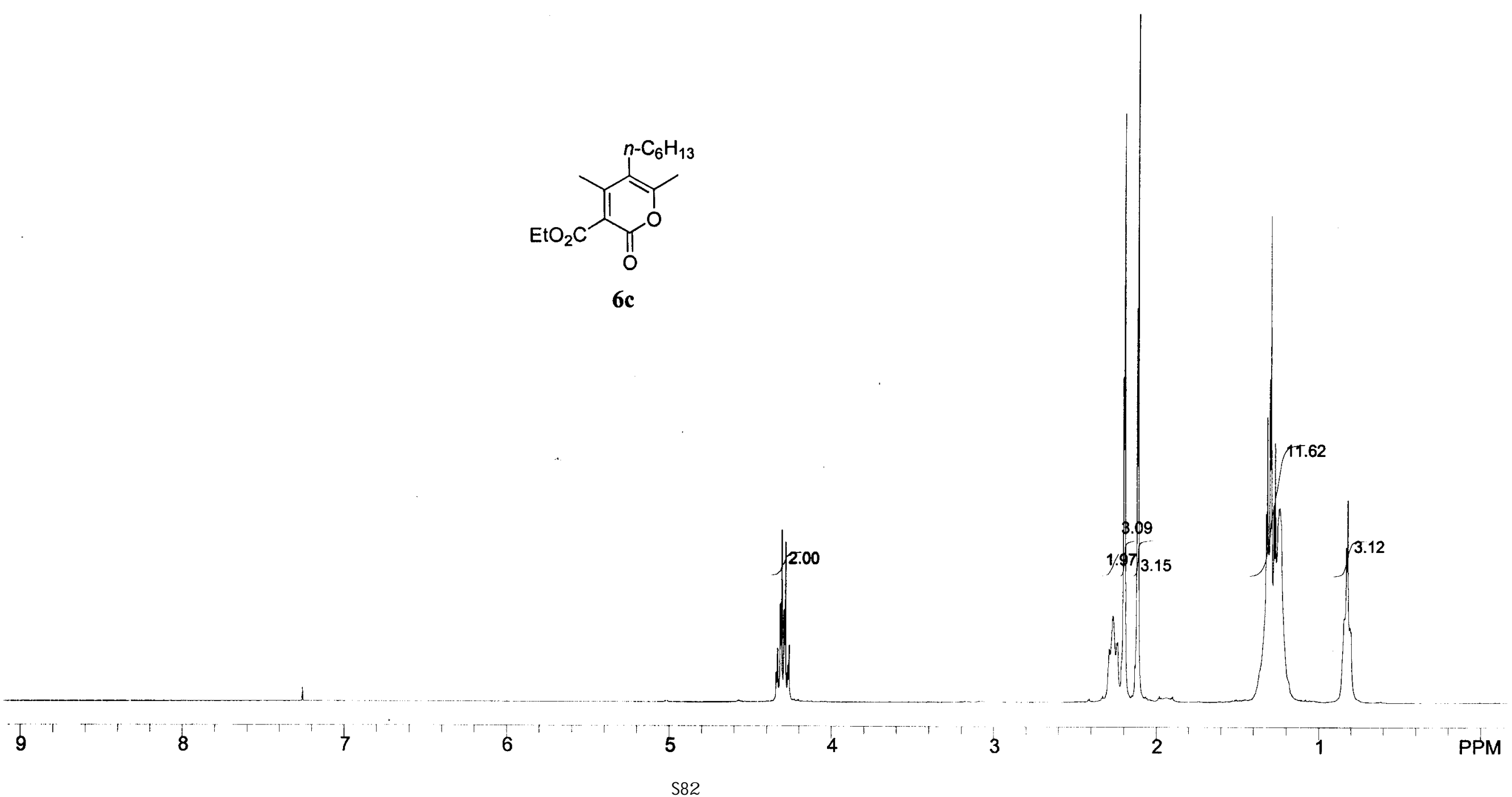

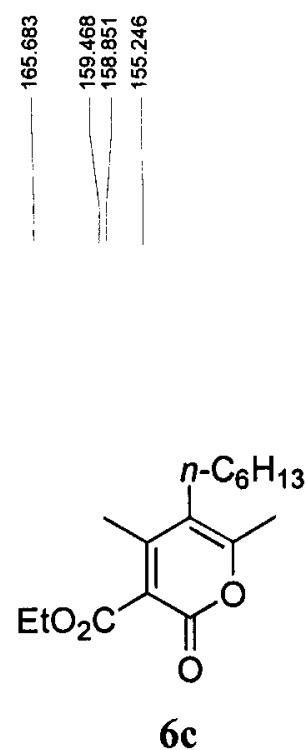

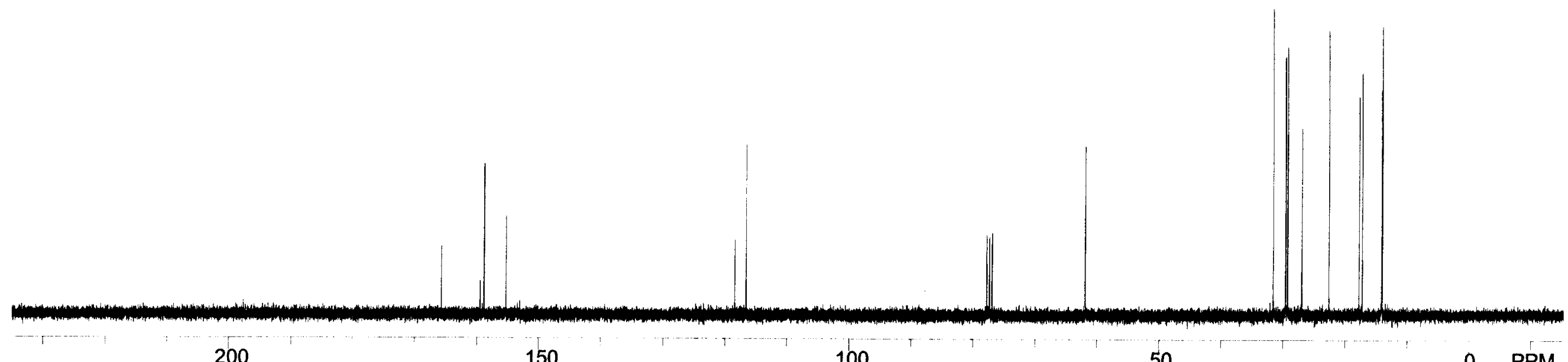


m|m|Om|

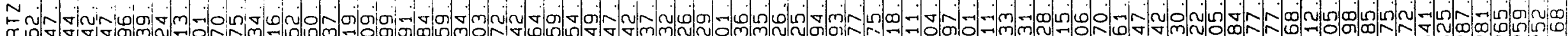
w.

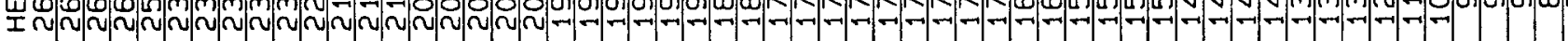

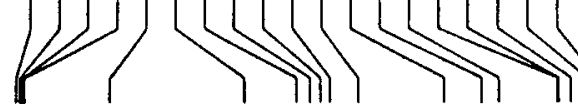

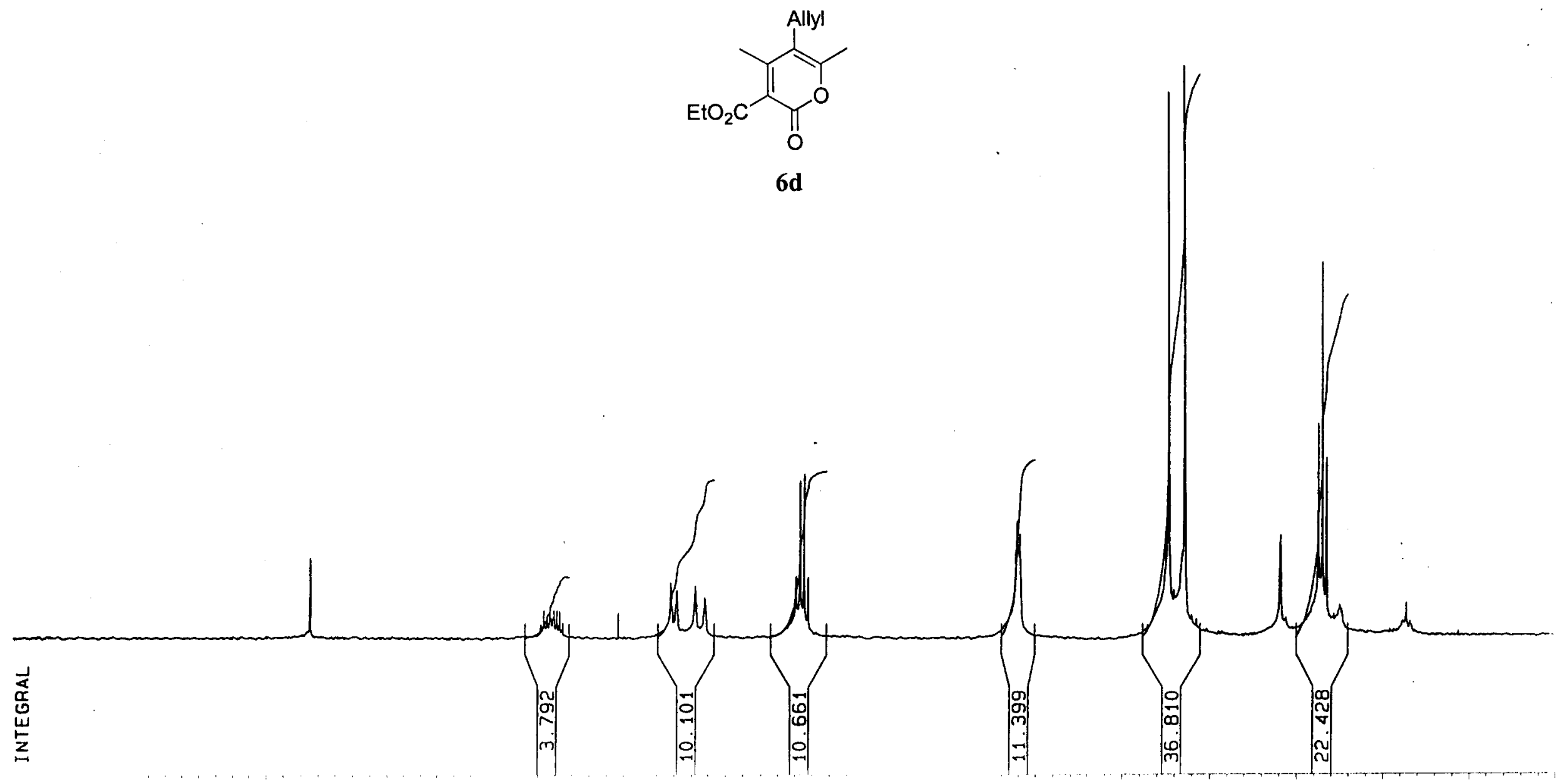




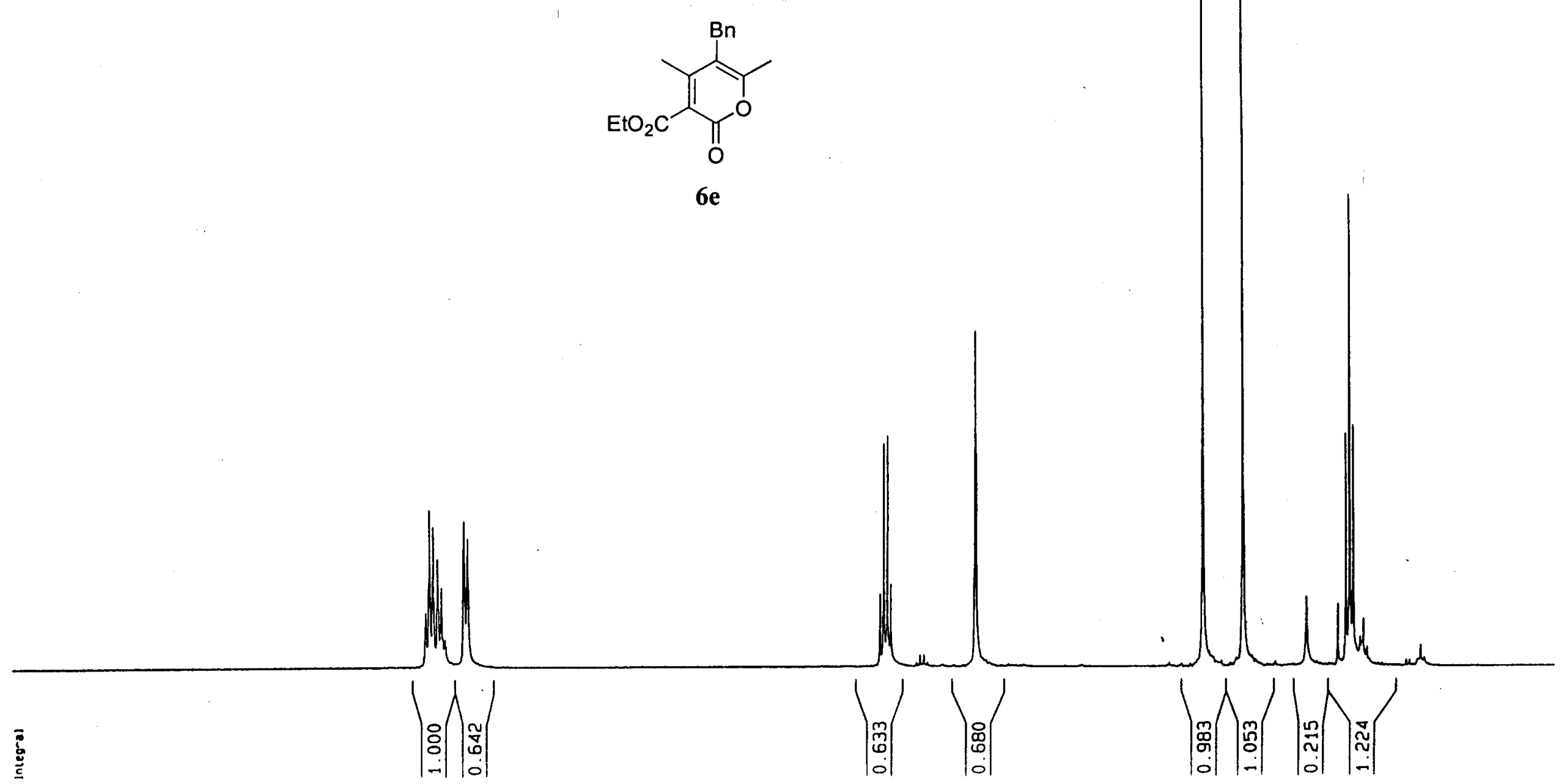




定

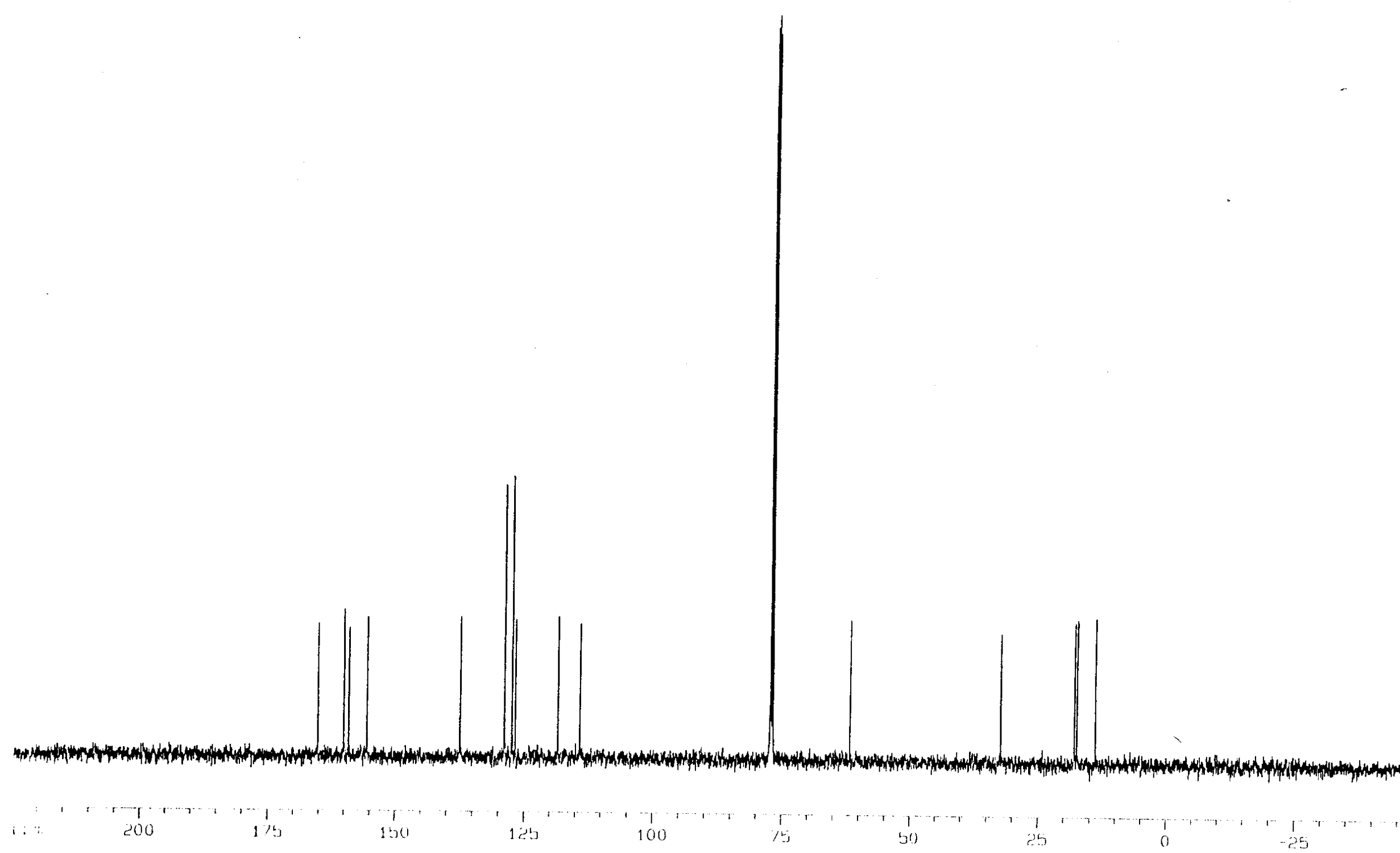



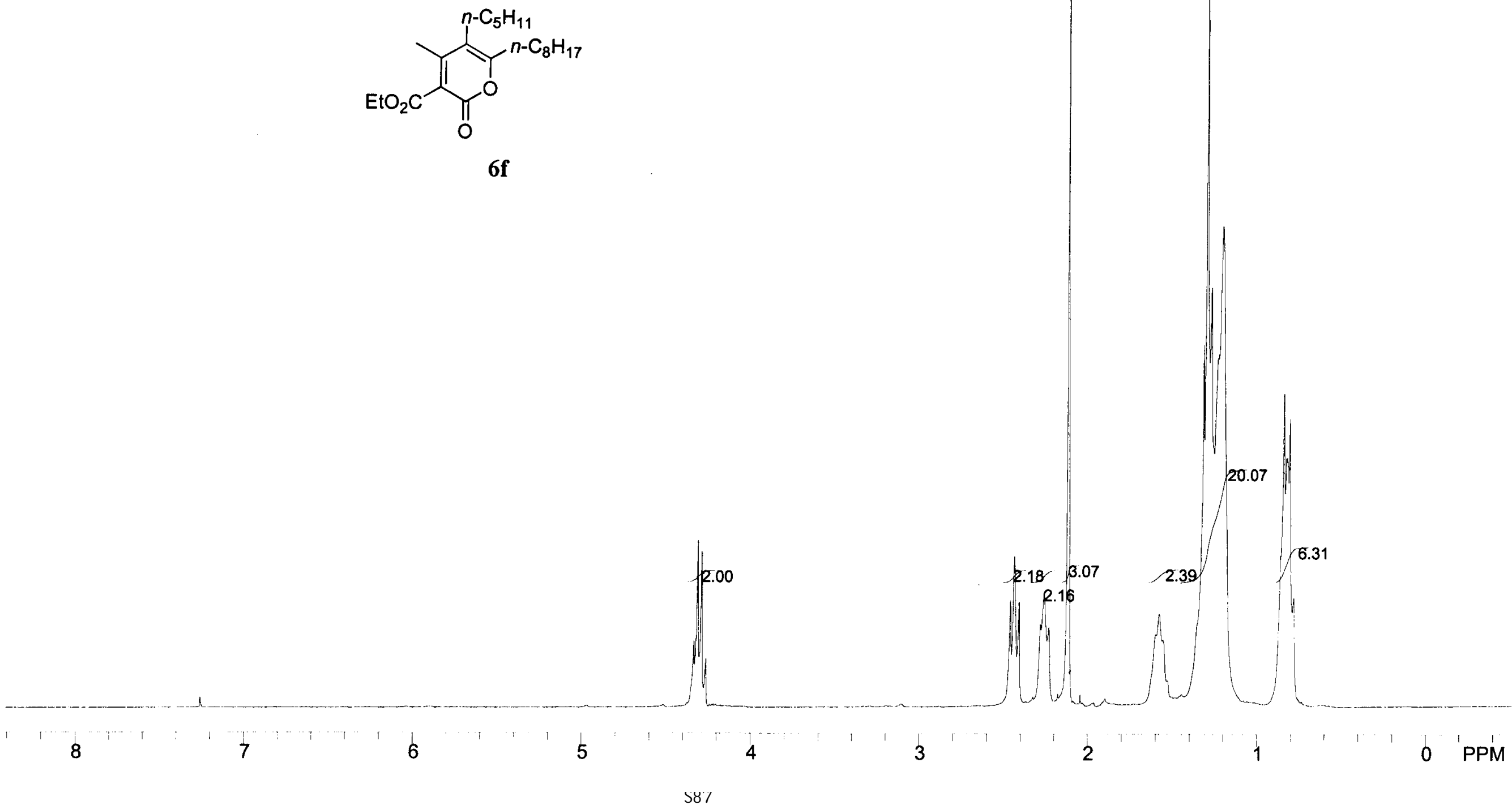


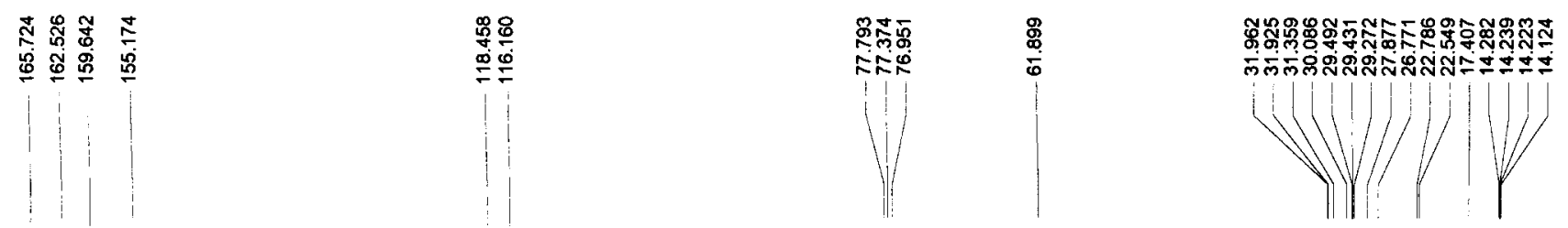
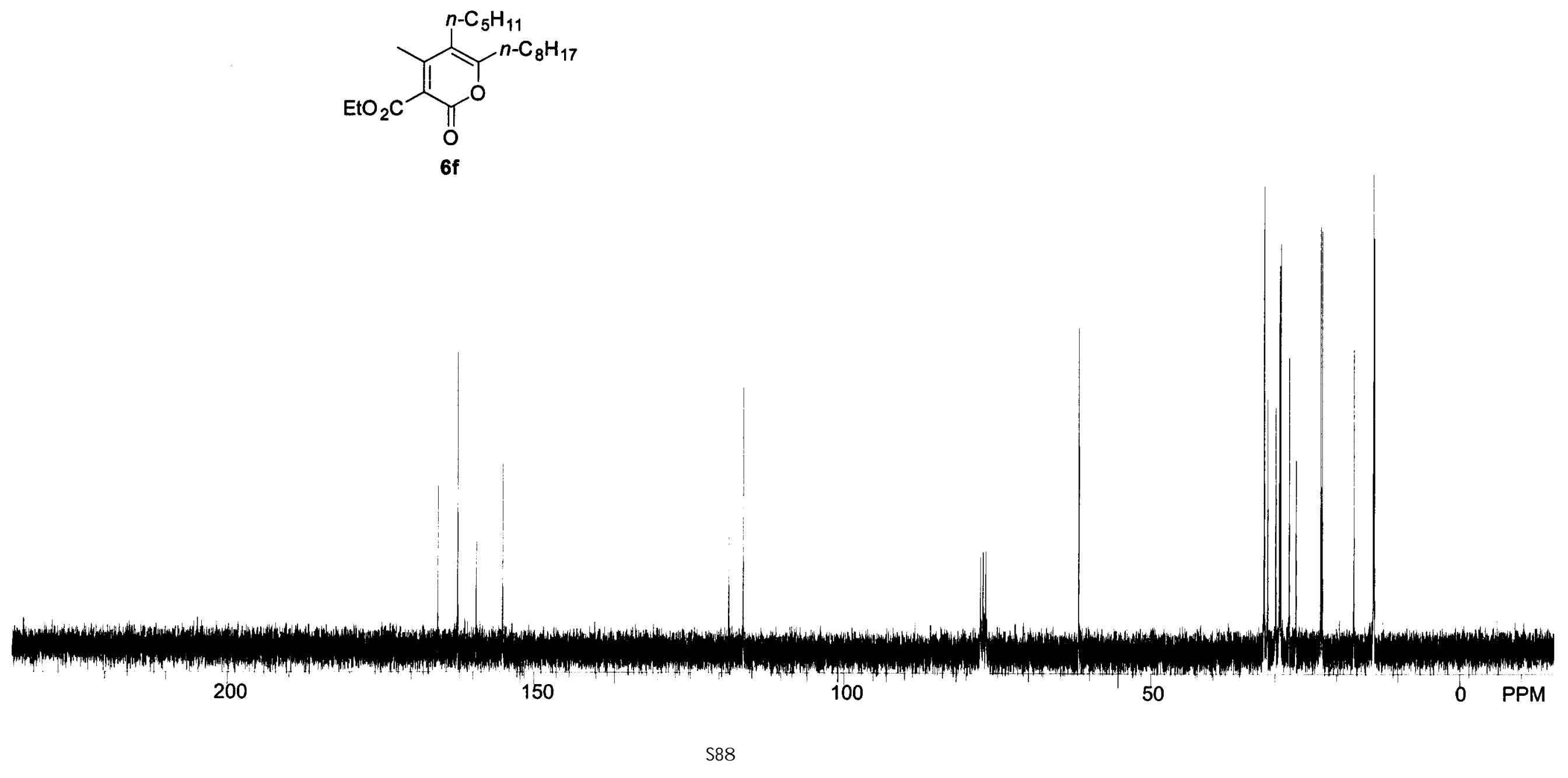

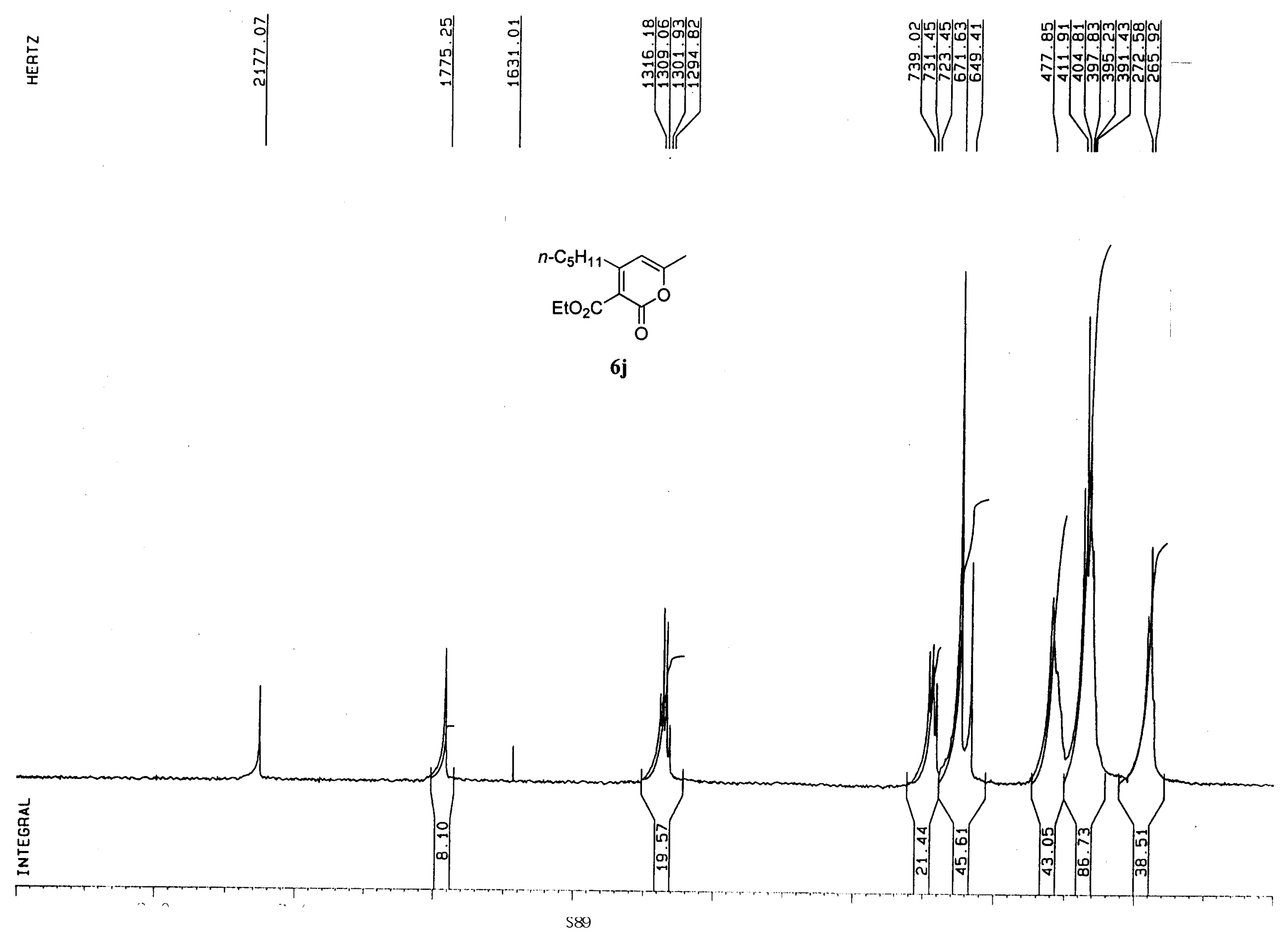

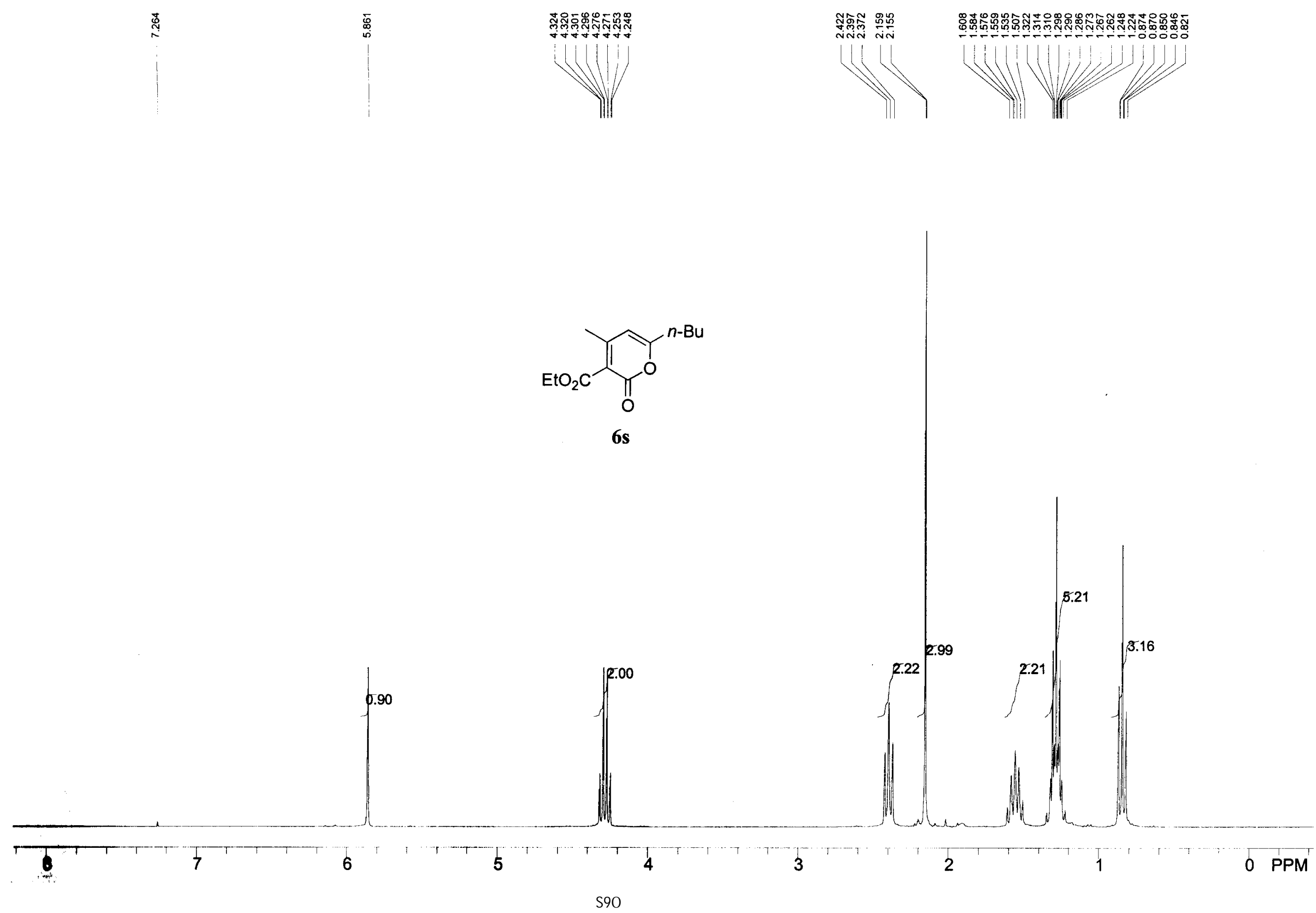


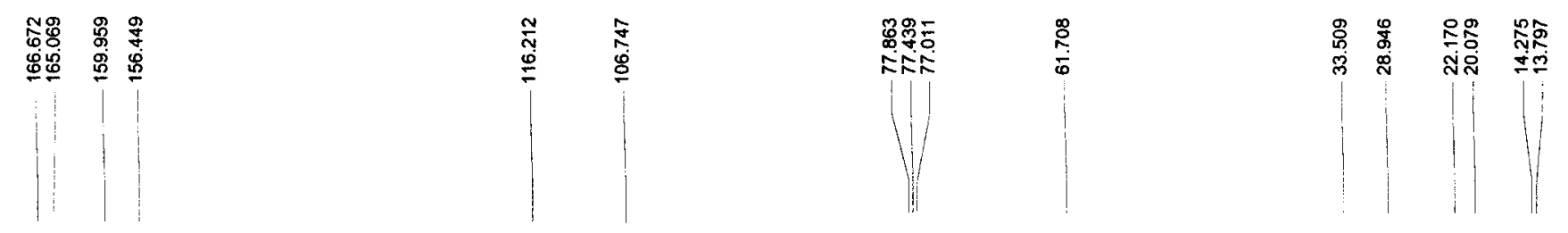

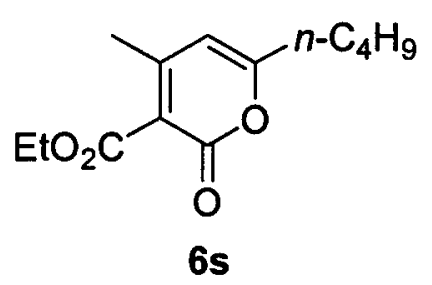




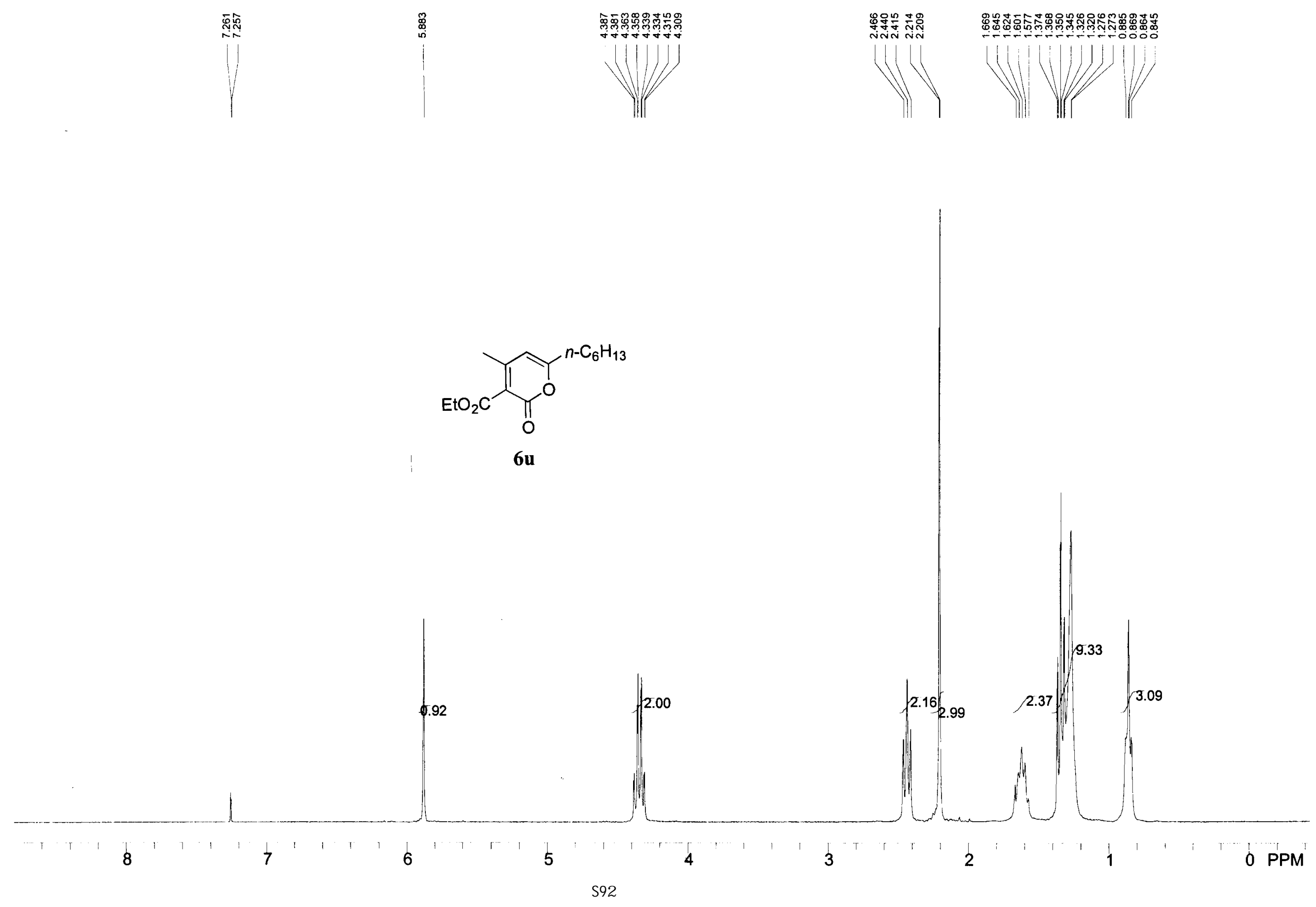




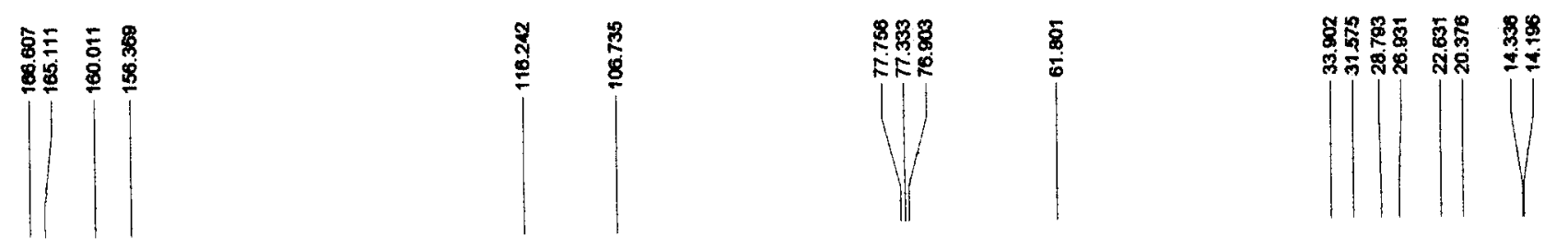
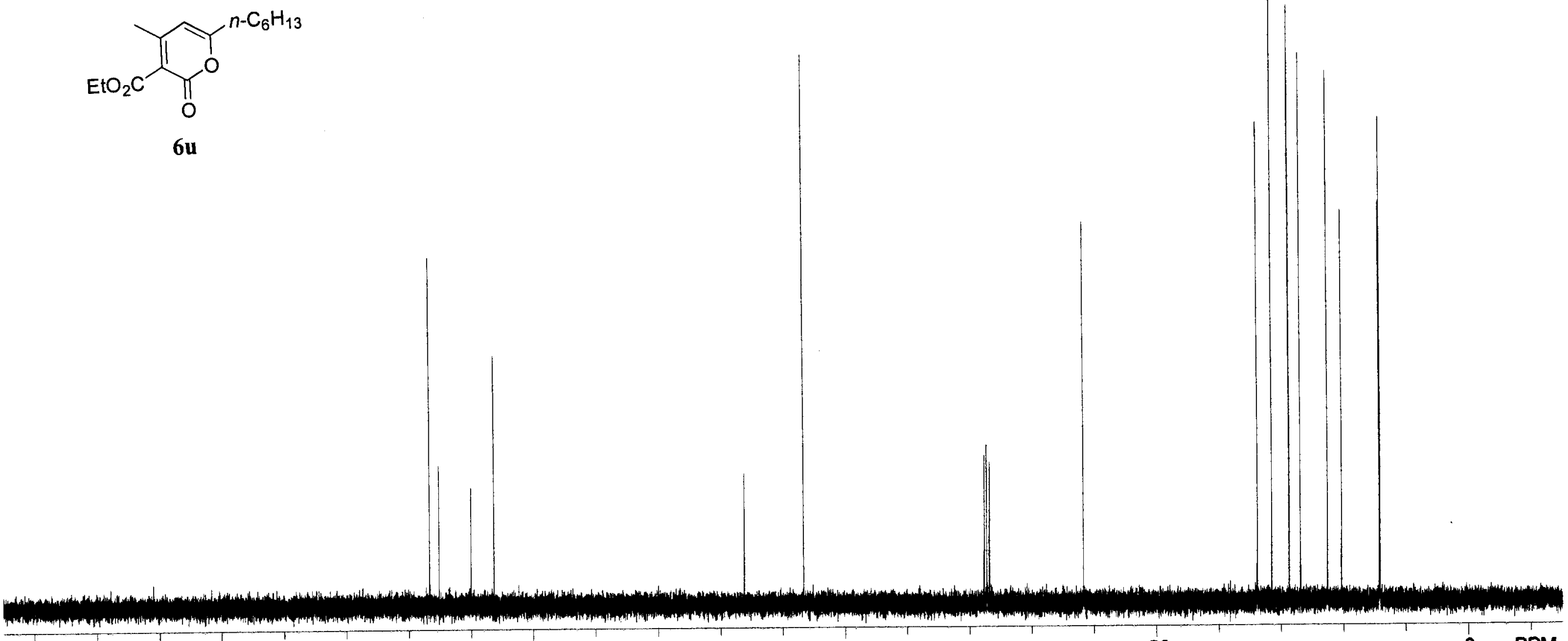

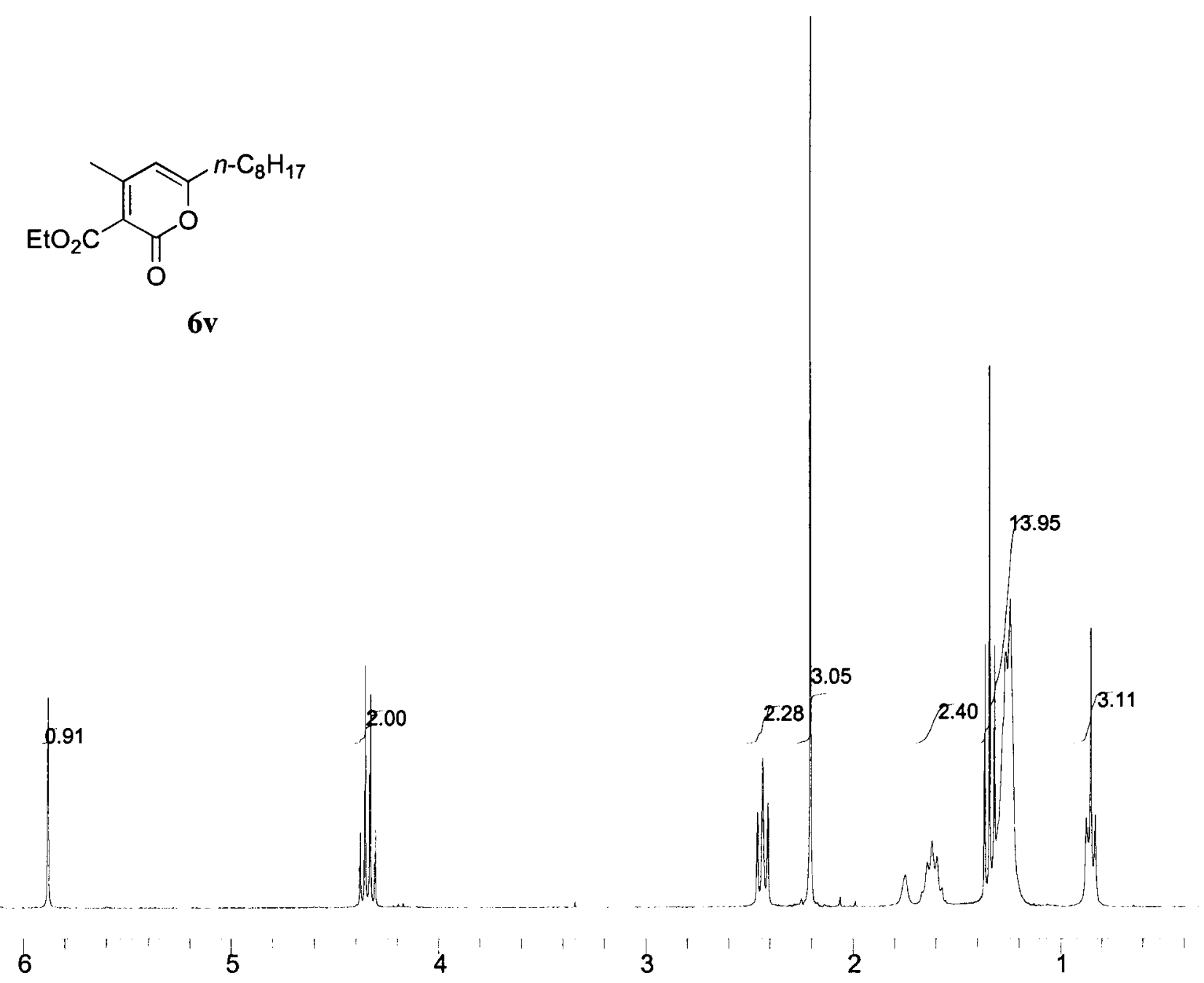

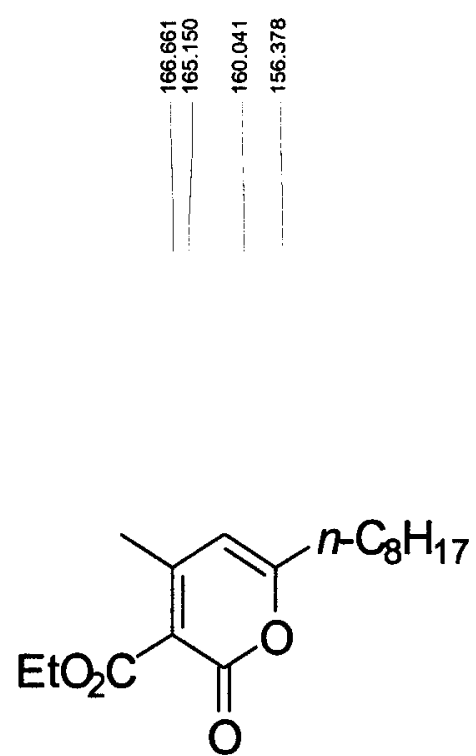

6v

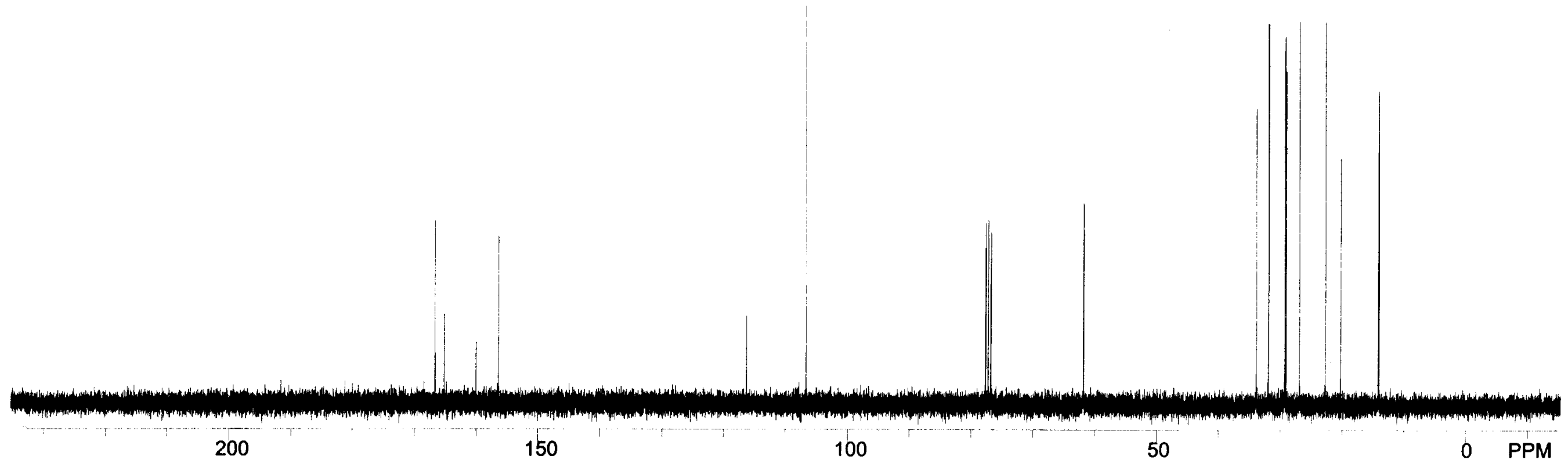




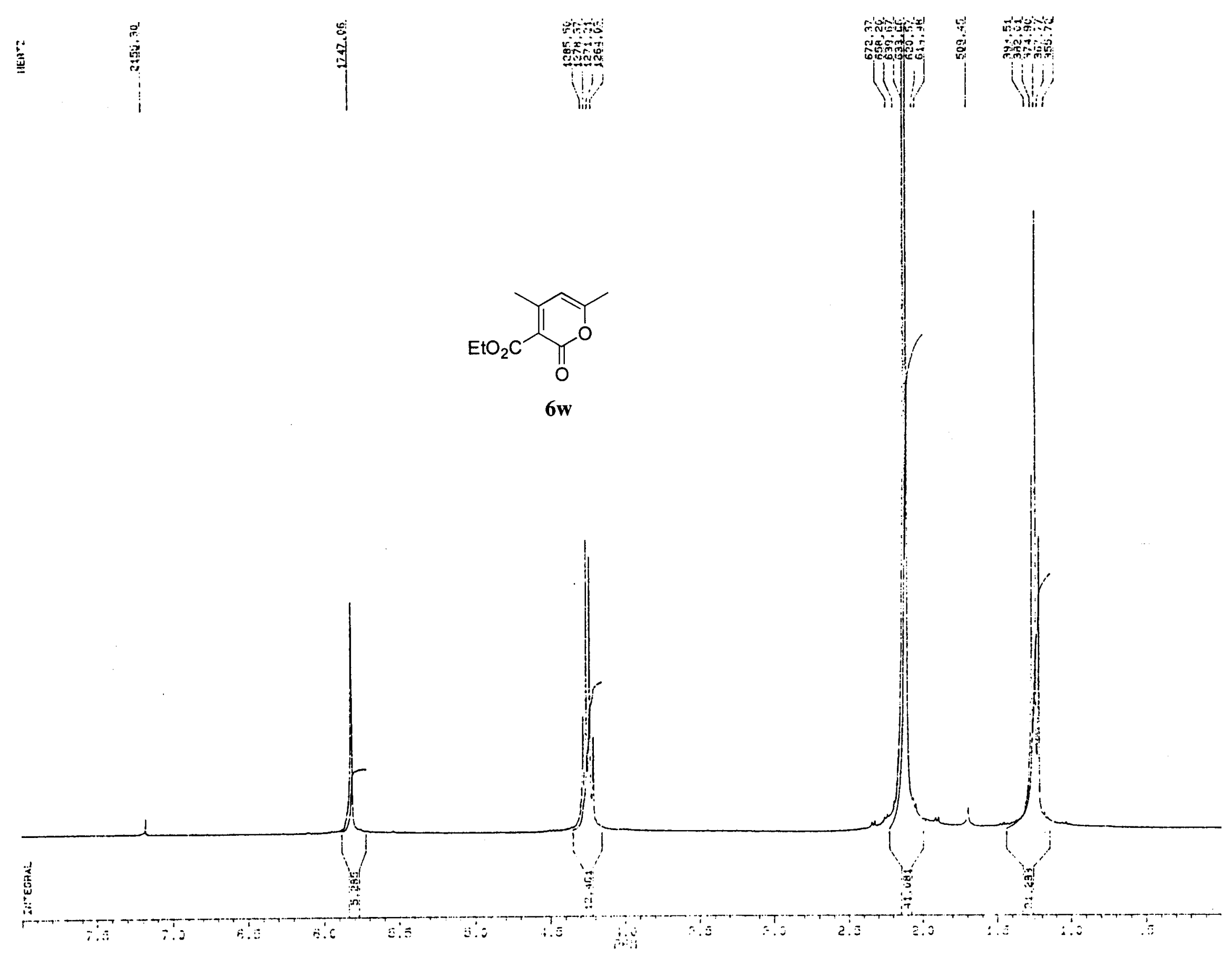




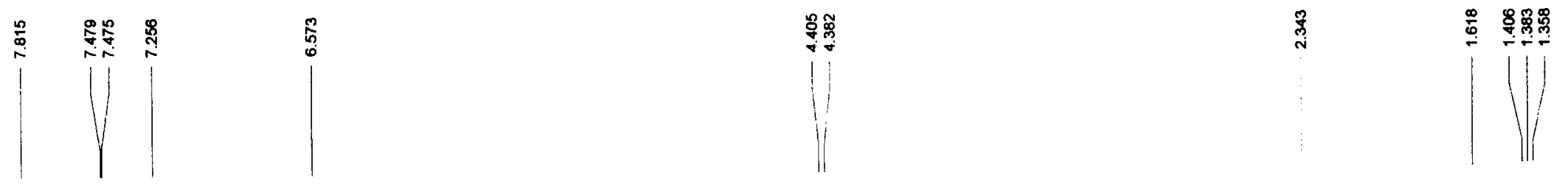

YSH-3-24-1
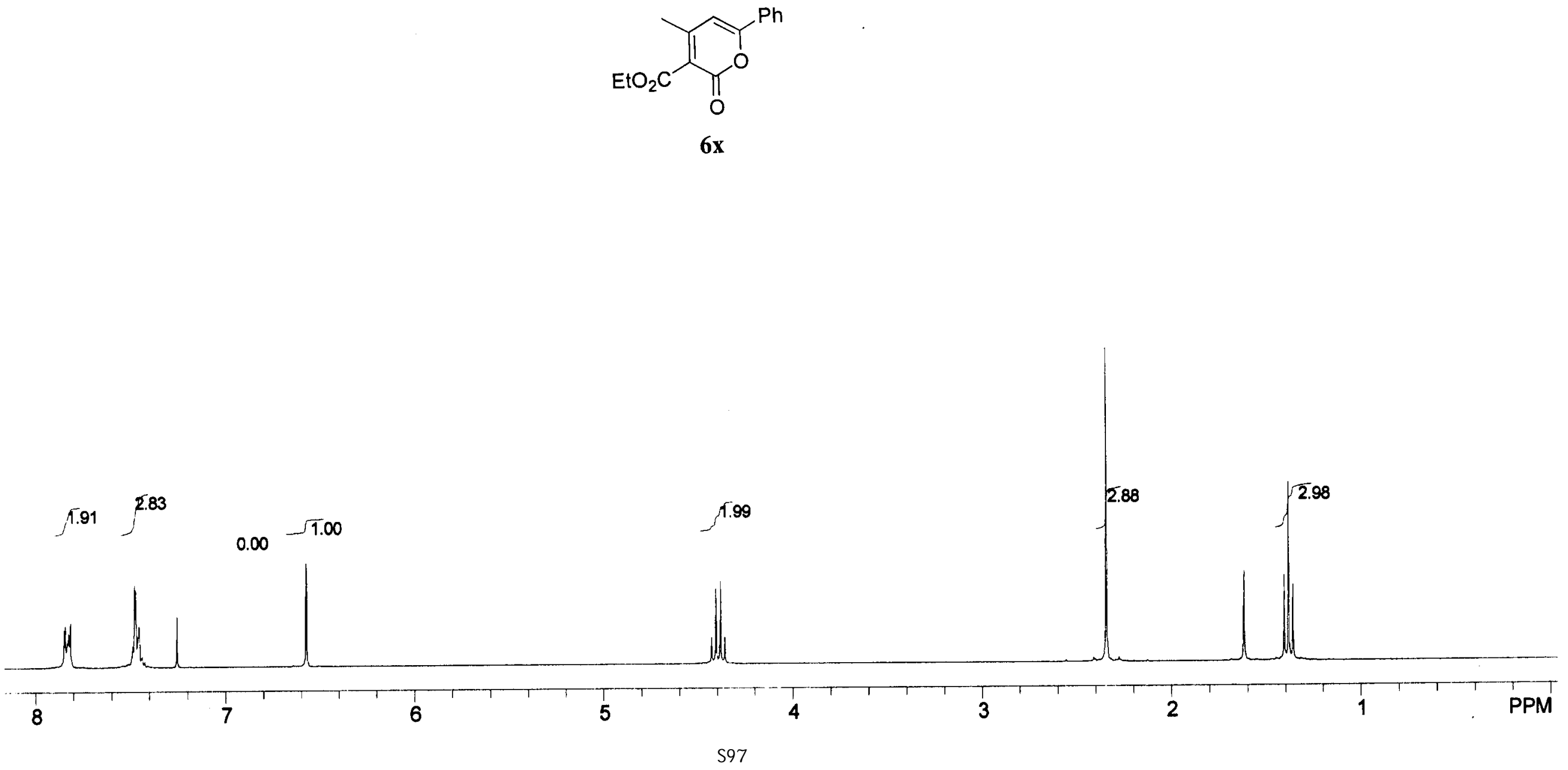

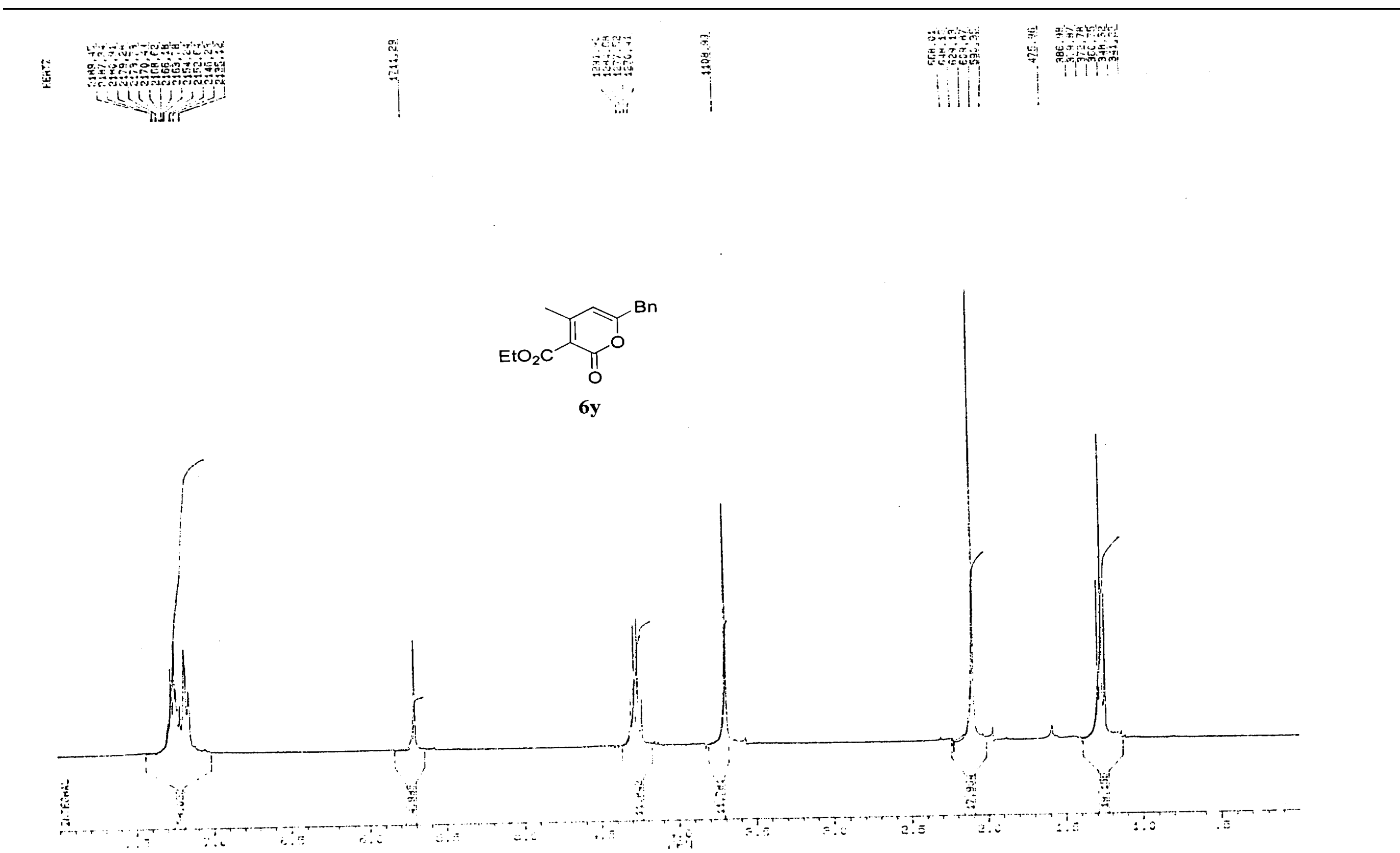